\title{
Oligodendroglial anillin facilitates septin assembly to prevent myelin outfoldings
}

\author{
Dissertation \\ for the award of the degree \\ "Doctor rerum naturalium" (Dr. rer. nat) \\ of the Georg-August University Göttingen \\ within the doctoral program Biology \\ of the Georg-August University School of Science (GAUSS) \\ submitted by \\ Michelle Scarlett Erwig \\ from Neuss, Germany
}

Göttingen, November 2018 


\section{Members of the Examination Board:}

Thesis committee:

PD Dr. Hauke Werner (Reviewer)

Department of Neurogenetics

Max Planck Institute of Experimental Medicine

Prof. Dr. Siegrid Löwel (Reviewer)

Department of Systems Neuroscience

Georg-August University, Göttingen

\section{Further members of the Examination Board:}

Prof. Dr. Martin Göpfert

Department of Cellular Neurobiology

Schwann-Schleiden Research Centre

Georg-August University, Göttingen

Prof. Dr. Ralf Heinrich

Department of Cellular Neurobiology

Schwann-Schleiden Research Centre

Georg-August University, Göttingen

Prof. Dr. Dr. Hannelore Ehrenreich

Department of Clinical Neuroscience

Max Planck Institute of Experimental Medicine

Prof. Dr. Alexander Flügel

Institute for Neuroimmunology and Multiple Sclerosis Research

University Medical Center Göttingen

Date of oral examination: January $28^{\text {th }}, 2019$ 


\section{Declaration}

I hereby declare that the Ph.D. thesis entitled "Oligodendroglial anillin facilitates septin assembly to prevent myelin outfoldings", has been written independently and with no other sources and aids than quoted.

Göttingen, November 27 $7^{\text {th }}, 2018$

Michelle Erwig 
Für meine Eltern. 


\section{Danksagung}

Ich möchte Prof. Klaus-Armin Nave Ph.D. danken, dass ich in seiner Abteilung arbeiten konnte. Danke für wissenschaftliche Diskussionen und eine Arbeitsatmosphäre in der alle auf einem Level diskutieren können.

Ein großer Dank geht an PD Dr. Hauke Werner. Danke für die Betreuung der Arbeit und die Zusammenarbeit. Die wissenschaftlichen Diskussionen sowie die familiäre Arbeitsatmosphäre aber auch das Ermutigen, sich weiter zu entwickeln, werden mir immer in guter Erinnerung bleiben.

Des Weiteren möchte ich Prof. Dr. Sigrid Löwel dafür danken, dass sie das Amt der zweiten Betreuerin übernommen hat. Danke für hilfreiche Diskussionen in den TCMs und die unkomplizierte Betreuung.

Ein weiteres Danke geht an Dr. Julia Patzig, die dieses Projekt begonnen hat und mir so viel beigebracht hat.

Ulrike Bode, Annette Fahrenholz, Torben Ruhwedel, Christos Naidis und Uschi Kutzke möchte ich für all die technische Hilfe danken.

Danke an Dr. Wiebke Möbius und Dr. Anna Steyer für Hilfe aller Art in der Elektronenmikroskopie. Danke, dass inr euer Wissen mit mir geteilt habt und auf so wunderbare Weise zu dieser Arbeit beigetragen habt.

Danke an unsere Kollaboratoren: Dr. Olaf Jahn für die Proteomanalyse und Hilfe bei angefallenen Fragen; Prof. Dr. Ingo Heilmann für die PIP 2 Messungen; Dr. Payam Dibaj für die elektrophysiologischen Messungen. Danke für die reibungslose und unkomplizierte Zusammenarbeit.

Ebenfalls ein großer Dank geht an Dr. Kathrin Kusch. Danke für deine Hilfe, deine Geduld und, dass du dein Wissen mit mir geteilt hast.

Von Herzen danken möchte ich Ramona Jung. Danke für all deine Hilfe ob während der Elternzeit oder wenn sonst etwas schief gegangen ist.

Ein großer Dank geht an die AG Werner. Es war eine schöne Zeit mit euch zu arbeiten, zu diskutieren, zu singen, zu tanzen, zu kochen \& essen und immer wieder auch zu lachen.

Ein extra Danke geht an Lena, Lisa und Maria. Unsere Diskussionen und eure Korrekturen haben zur Fertigstellung dieser Arbeit beigetragen.

Ein großer Dank geht an die Tierpfleger Marion Peine und Kerstin Claus. Danke, dass ihr immer ein Auge auf meine Tiere hattet und unsere Zusammenarbeit so reibungslos verlief. Eure Arbeit war die Grundlage für all meine Experimente.

Danke ebenfalls an die glorreichen 6. 2007 haben wir unser Abenteuer in Göttingen begonnen und den Entschluss gefasst, dieses eine Mädchen zu küssen. Aus Fremden sind dabei Freunde geworden; danke, dass ihr immer für mich da seid.

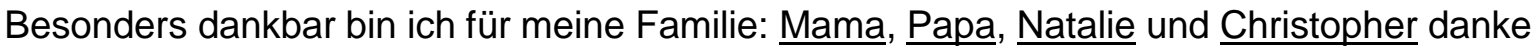
für eure Unterstützung und all die Liebe.

Von ganzem Herzen möchte ich Jan Erwig danken. Du hast immer an mich geglaubt und mir immer den Rücken gestärkt. Danke, dass du mich verstehst, so geduldig bist und mich so liebst wie ich bin. Danke für unsere wunderbare Tochter und dafür, dass du Karlotta der beste Papa der Welt bist. 


\section{Content}

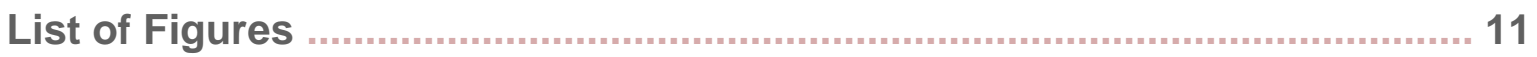

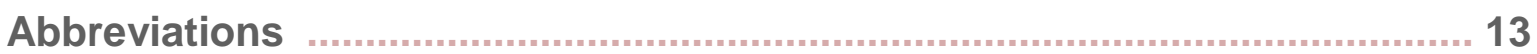

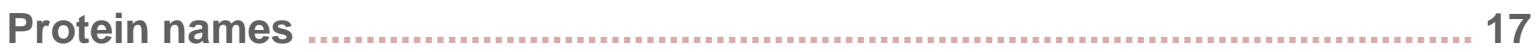

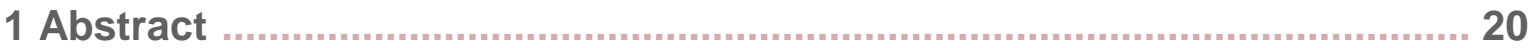

2 Introduction $\ldots \ldots \ldots \ldots \ldots \ldots \ldots \ldots \ldots$

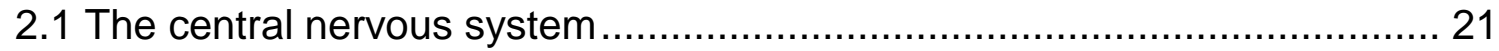

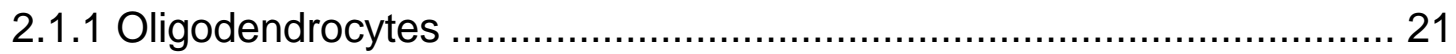

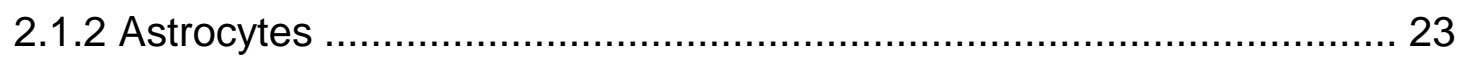

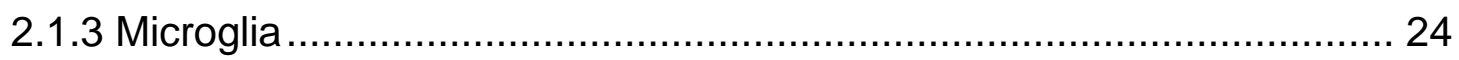

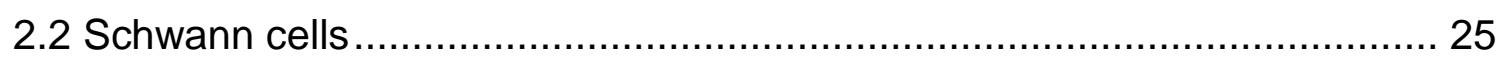

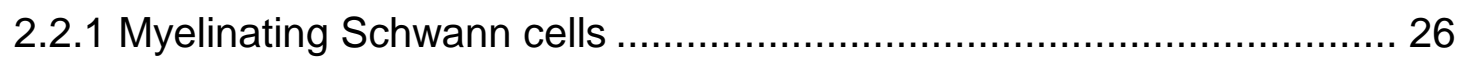

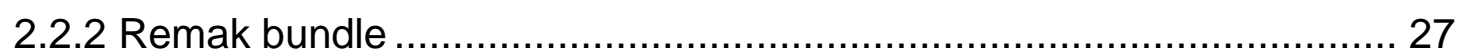

2.3 Myelin integrity and pathological myelin outfoldings ................................ 27

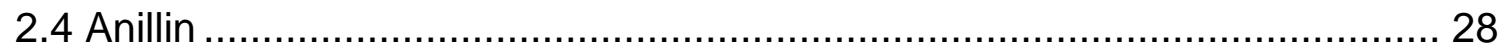

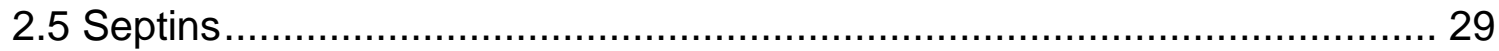

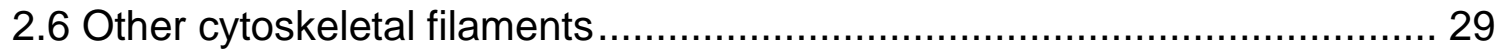

2.7 Axon caliber

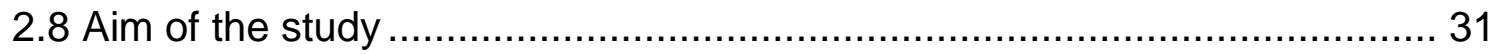

3 Materials and Methods $\ldots \ldots \ldots \ldots \ldots$

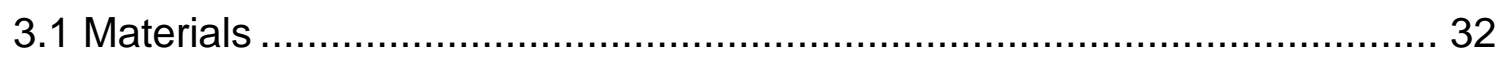

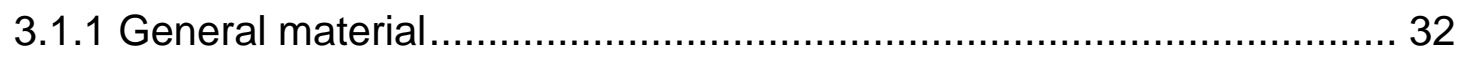

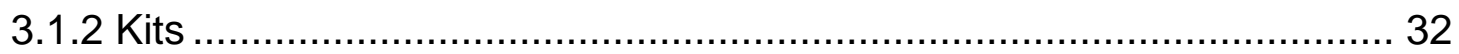

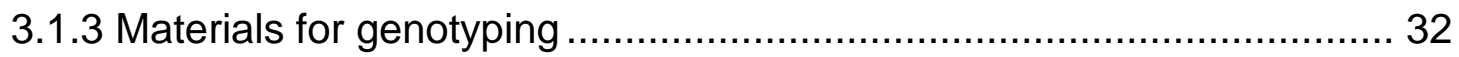

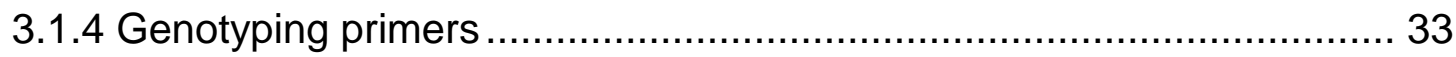

3.1.5 Quantitative real-time PCR primers................................................. 34

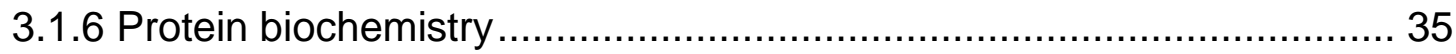




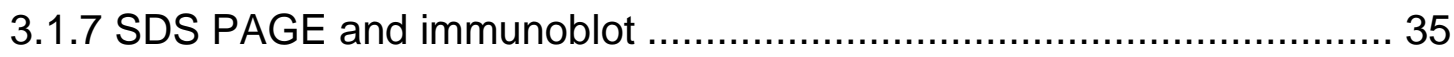

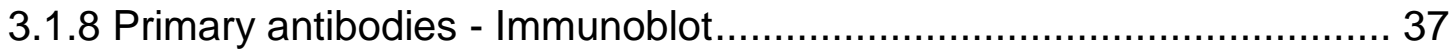

3.1.9 Secondary antibodies - Immunoblot .............................................. 38

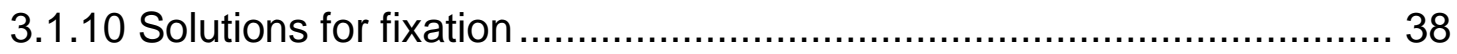

3.1.11 Immunohistochemistry and staining solutions ................................. 39

3.1.12 Primary antibodies - Immunohistochemistry.................................. 41

3.1.13 Secondary antibodies - Immunohistochemistry .............................. 41

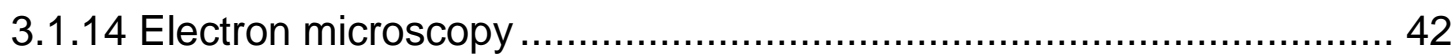

3.1.15 Solutions for silver impregnation of protein gels ............................. 42

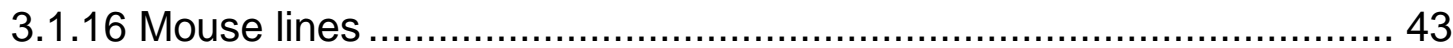

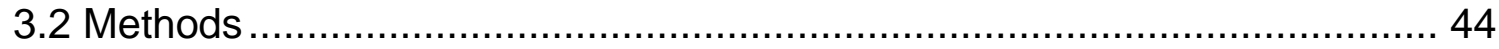

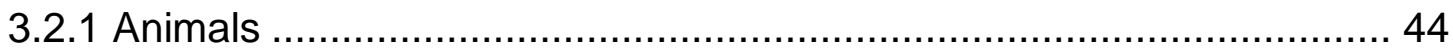

3.2.2 Generation of Anln conditional knockout mice (Anln cKO) .................. 44

3.2.3 Generation of Sept9R88W/Wt mice ..................................................... 44

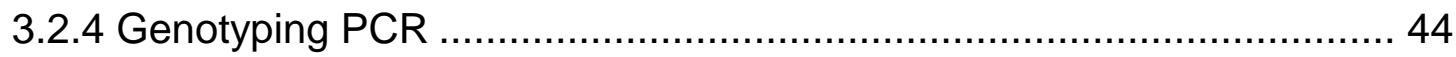

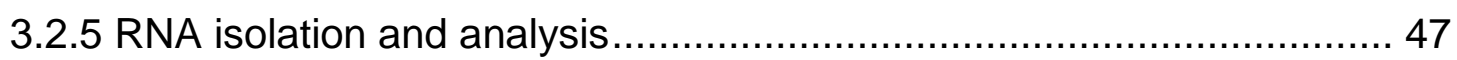

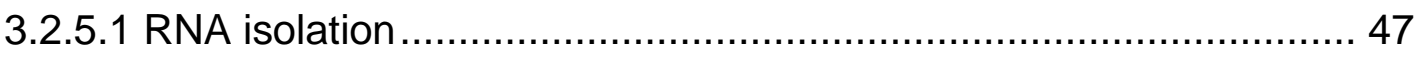

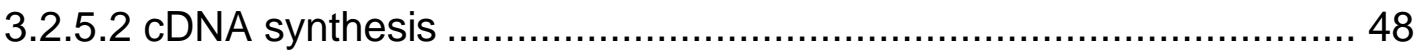

3.2.5.3 Quantitative real-time PCR (qRT-PCR) …………………......... 49

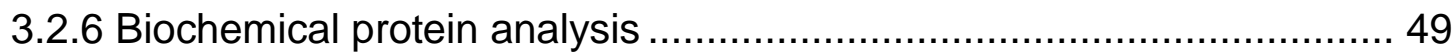

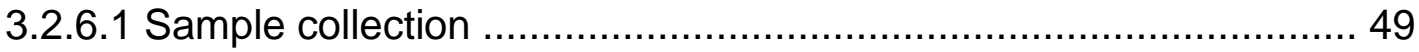

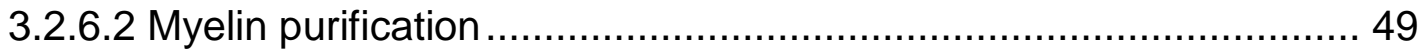

3.2.6.3 Lysate preparation (PNS) …………………........................ 50

3.2.6.4 Protein concentration determination and sample preparation......... 50

3.2.6.5 Protein separation using SDS PAGE .......................................... 51

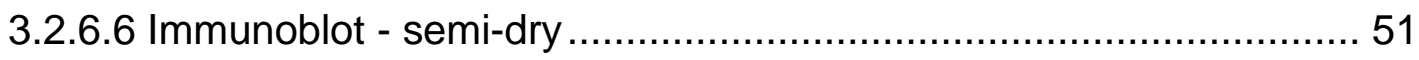

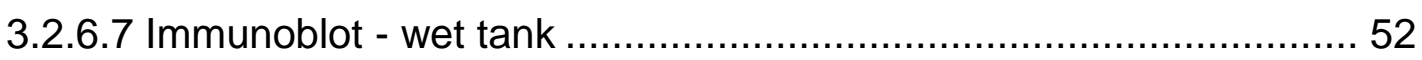

3.2.6.8 Immunodetection of blotted proteins........................................... 52 
3.2.6.9 Quantitative Mass Spectrometry ............................................... 52

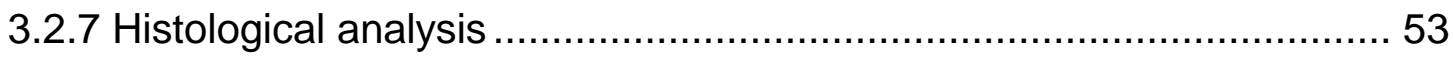

3.2.7.1 Perfusion fixation of mouse tissue .............................................. 53

3.2.7.2 Embedding for cryosections .................................................... 53

3.2.7.3 Immunohistochemistry - cryosections........................................... 53

3.2.7.4 Imaging and analysis of fluorescent staining ................................ 54

3.2.7.5 Embedding for paraffin sections .............................................. 55

3.2.7.6 Immunohistochemistry - paraffin sections.................................... 55

3.2.7.7 Imaging and quantification of DAB developed stainings ................ 57

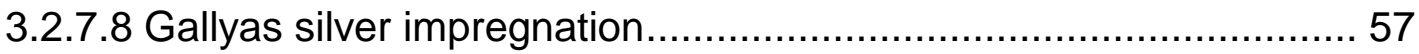

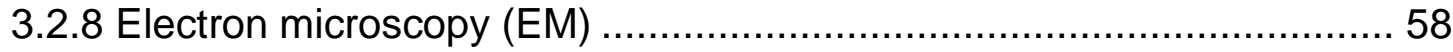

3.2.8.1 Tissue preparation - Electron microscopy.................................... 58

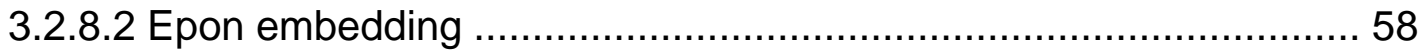

3.2.8.3 Sectioning of Epon embedded tissue ………............................... 59

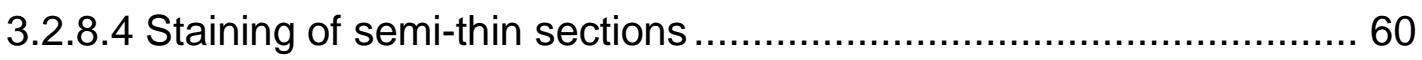

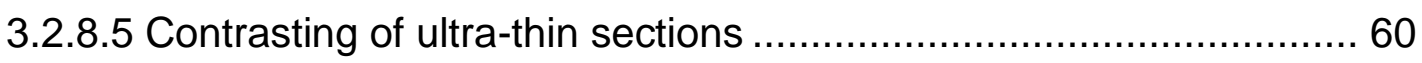

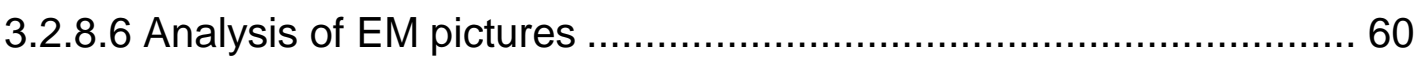

3.2.9 Focused ion beam scanning electron microscopy (FIB SEM) .............. 63

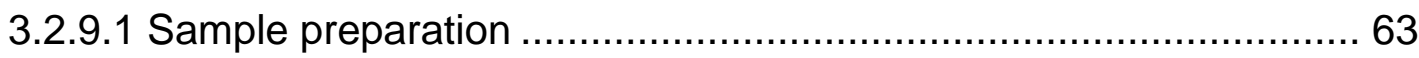

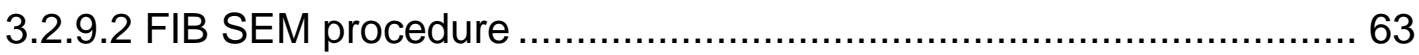

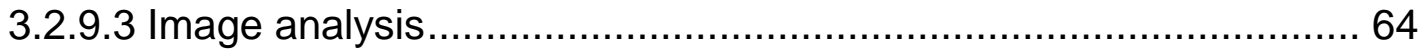

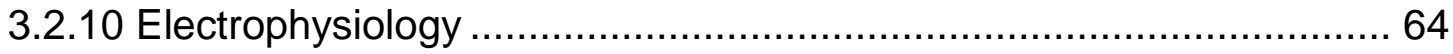

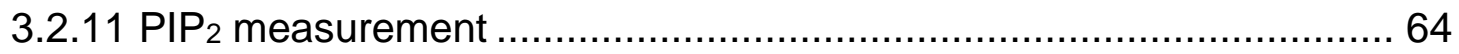

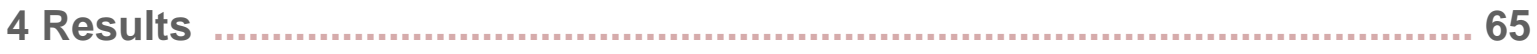

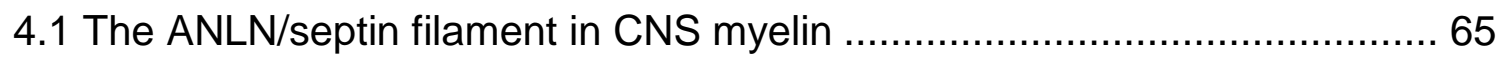

4.1.1 Anillin and septins co-localize in CNS white matter............................. 65

4.1.2 ANLN and septins appear similar in development.............................. 67

4.2 Characterization of An/ $n^{f / f t} ; C n p^{C r e / W t}$ mice .............................................. 67 
4.2.1 Generation of mice lacking ANLN from myelinating glia cells

4.2.2 Mass spectrometric comparison of Anln cKO mice and controls..........68 68

4.2.3 Loss of myelin septins upon conditional depletion of ANLN ............... 71

4.2.4 mRNA abundance of regulated proteins ................................... 72

4.2.5 Myelin abnormalities......................................................... 73

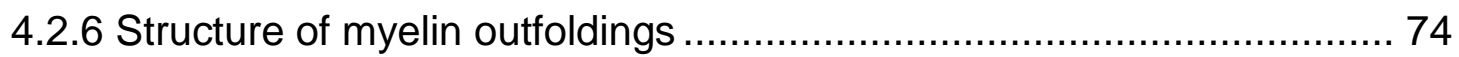

4.2.7 Decelerated nerve conduction velocity in Anln cKO mice ................... 75

4.2.8 Unaltered brain structure and myelination in AnIn cKO mice .............. 76

4.2.9 The loss of ANLN has no impact on myelin thickness ....................... 77

4.2.10 No signs of secondary neuropathology in CNS white matter ............ 78

4.2.11 Anln cKO mice display reduced PIP 2 levels ................................ 81

4.2.12 Axon diameter frequency distribution ..................................... 81

5 Discussion

5.1 Is ANLN influencing septin filament assembly? ................................. 85

5.2 ANLN/septin filaments in development - who comes first? ...................... 86

5.3 Do myelin outfoldings decrease nerve conduction velocity? ................... 87

5.4 Does loss of ANLN lead to altered $\mathrm{PIP}_{2}$ levels in CNS myelin? ................. 88

5.5 Myelin stability; a novel effector of axon diameter restriction? .................. 89

6 References

7 Addendum

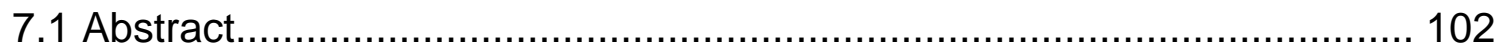

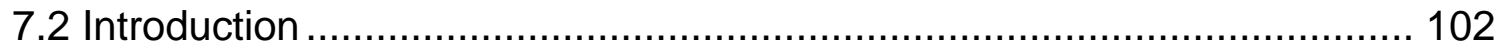

7.2.1 Septins in the peripheral nervous system.................................. 102

7.2.2 Hereditary neuralgic amyotrophy......................................... 103

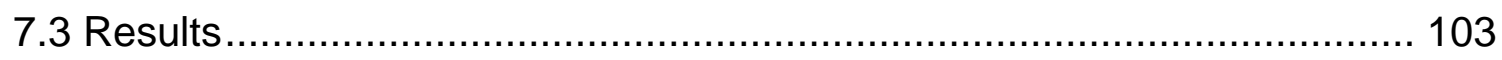

7.3.1 Analysis of SeptgR88W/Wt mice ............................................ 103

7.3.1.1 Point mutation has no effect on septin protein abundances ......... 103

7.3.1.2 No changes in localization of SEPT9 ................................. 104 
7.3.1.3 Unaltered nerve structure 105

7.3.1.4 Normal myelin ultrastructure 106

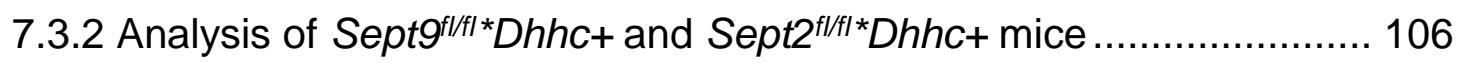

7.3.2.1 No alterations in axon sorting at P4 ……............................... 106

7.3.2.2 Number of myelinated axons are unchanged at P14 .................. 108

7.3.2.3 Normal myelination state at 6 mo of age ................................... 109

7.3.2.4 No changes in axon diameters Sept9f/ff*$D h h c+$ mice .................. 109

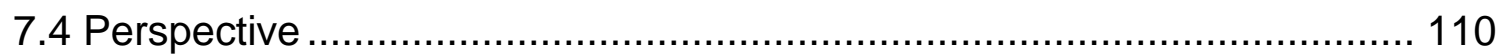




\section{List of Figures}

Figure 1 Theoretical relation between conduction velocity and axon diameter

Figure 2 Illustration of compact and non-compact myelin in optic nerve cross section

Figure 3 Structure of CNS myelinated axons

Figure $4 \quad$ Neuroprotection or neurodegeneration induced by microglia

Figure 5 Schwann cell types in the PNS

Figure 6 Structure of PNS myelinated axons

Figure 7 RNA-Seq analysis indicates Anln mRNA expression in oligodendrocytes

Figure $8 \quad$ Overview of profiles found in electron micrographs

Figure 9 Localization of the ANLN/septin filament in mouse CNS

Figure 10 ANLN and septins in development

Figure 11 Generation of $A n / n^{f / f t} ; C n p^{C r e / W t}$ mice

Figure 12 Mass spectrometric analysis of purified myelin

Figure 13 Abundance of ANLN, septins, and classical myelin proteins

Figure 14 qRT-PCR analysis of regulated proteins

Figure 15 Lack of ANLN/septin filament causes myelin outfoldings

Figure 163 3D reconstruction of myelin outfoldings

Figure 17 Nerve conduction velocity and node structure

Figure 18 CNS myelination and myelin periodicity

Figure 19 g-ratio and axon integrity

Figure 20 Lack of ANLN in oligodendrocytes does not lead to secondary neuropathology

Figure $21 \quad \mathrm{PIP}_{2}$ analysis in Anln cKO and control mice

Figure 22 Axon diameter frequency distribution in Anln cKO mice

Figure 23 Axon diameter frequency distribution in SEPT8 deficient and $C n p^{C r e / W t}$ mice 
Addendum Figure 1

Addendum Figure 2

Addendum Figure 3

Addendum Figure 4

Addendum Figure 5

Addendum Figure 6

Addendum Figure 7

Addendum Figure 8
Offspring analysis and abundance of SEPT9 and SEPT2 in Sept ${ }^{R 88 W / W t}$ mice

Localization of SEPT9 in sciatic nerves

Nerve structure and myelinated axons

Myelin ultrastructure

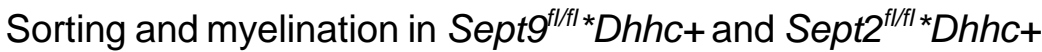
mice at P4

Myelinated axons at P14

g-ratio in Sept $9^{f / / f t *} D h h c+$ and Sept $2^{f / f t *} D h h c+$ mice

Axon diameter frequency distribution in Sept $9^{f / f t \mid}{ }^{*} D h h c+$ mice 


\section{Abbreviations}

Abbreviation

3D

Akt

Anln

Anln cKO

ANOVA

APS

BBB

bp

BSA

${ }^{\circ} \mathrm{C}$

cDNA

CKO

CLAHE

CNS

CRISPR

DAB

DAPI

$\mathrm{ddH}_{2} \mathrm{O}$

DDSA

DDT

Dhh

DMP-30

DNA

dNTP

DPSS

ECL

EDTA

e.g.

EM

Erk

ES

EUCOMM
Full form

Three dimensional

RAC-alpha serine/threonine-protein kinase (Protein kinase B)

Anillin (gene)

Anl/n ${ }^{f / f t} ; C n p^{C r e / W t}$

Analysis of variance

Ammonium persulfate

Blood brain barrier

Base pairs

Bovine serum albumin

Degrees Celsius

Complementary DNA

Conditional knockout

Contrast Limited Adaptive Histogram Equalization

Central nervous system

Clustered regularly interspaced short palindromic repeats

3,3'-Diaminobenzidine

4'-6-Diamidino-2-phenylindole

Double-distilled water

Dodecenylsuccinic anhydride

Dichlorodiphenyltrichloroethane

Desert hedgehog

2,4,6-Tris(dimethylaminomethyl)phenol

Deoxyribonucleic acid

Deoxyribonucleoside triphosphate

Diode-pumped solid-state

Enhanced chemiluminescence

Ethylenediaminetetraacetic acid

Exempli gratia

Electron microscopy

Extracellular signal-regulated kinase

Embryonic stem

European Conditional Mouse Mutagenesis Program 


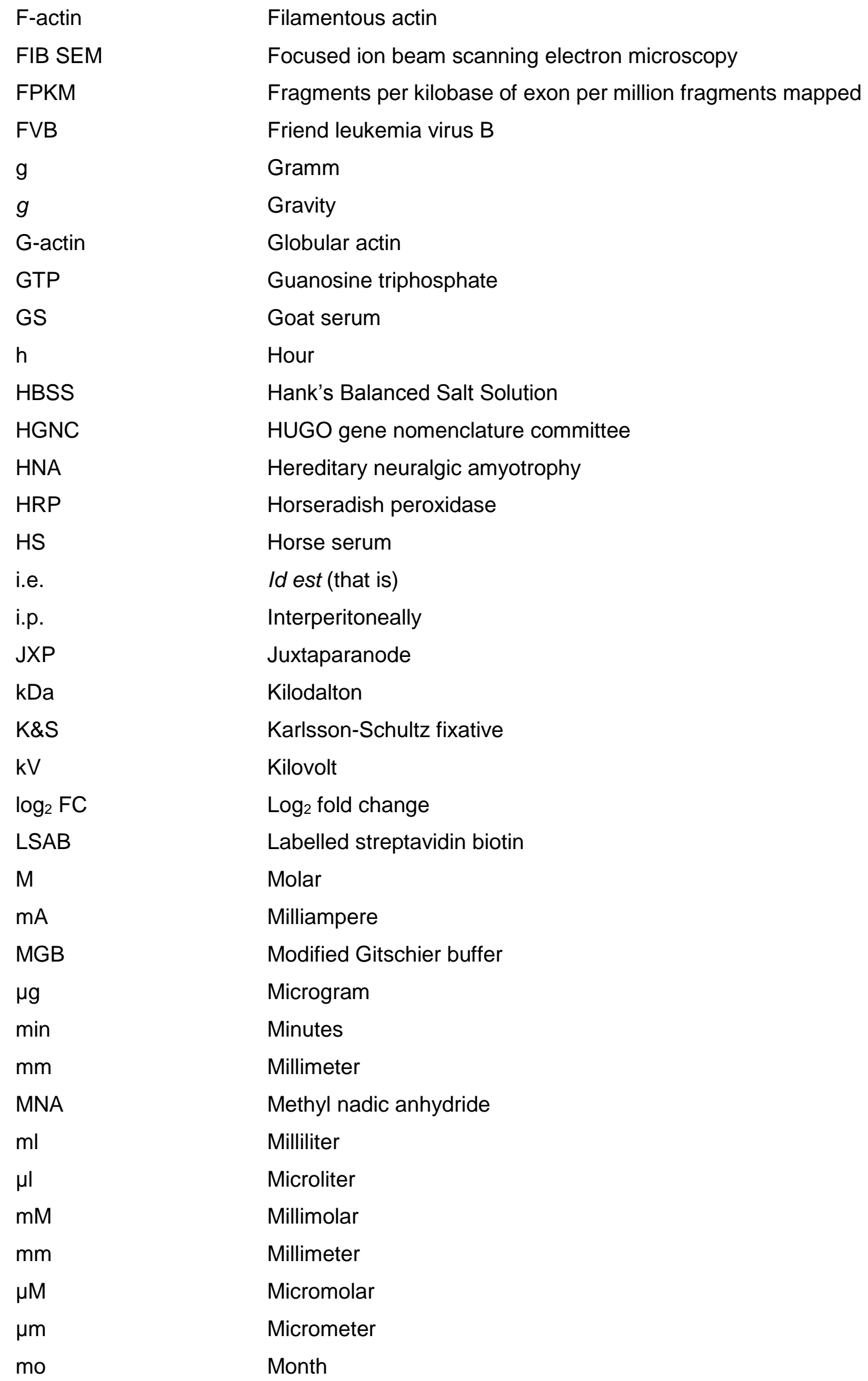

Gramm

Gravity

Globular actin

Guanosine triphosphate

Goat serum

Hour

Hank's Balanced Salt Solution

HUGO gene nomenclature committee

Hereditary neuralgic amyotrophy

Horseradish peroxidase

Horse serum

Id est (that is)

Interperitoneally

Juxtaparanode

Kilodalton

Karlsson-Schultz fixative

Kilovolt

$\log _{2}$ fold change

Labelled streptavidin biotin

Molar

Milliampere

Modified Gitschier buffer

Microgram

Minutes

Millimeter

Methyl nadic anhydride

Milliliter

Microliter

Millimolar

Millimeter

Micromolar

Micrometer

Month 


$\begin{array}{ll}\text { MPI EM } & \text { Max-Planck-Institute of Experimental Medicine } \\ \text { mRNA } & \text { Messenger RNA } \\ \text { MS } & \text { Mass spectrometry } \\ \text { mTOR } & \text { Rapamycin } \\ \text { N } & \text { Normality } \\ n & \text { Number (biological replicates) } \\ \text { nA } & \text { Nanoampere } \\ \text { ng } & \text { Nanogram } \\ \text { NgR1 } & \text { Nogo receptor 1 } \\ \text { nm } & \text { Nanometer } \\ \text { n.s. } & \text { Non-significant } \\ \text { o/n } & \text { Overnight } \\ \text { OPC } & \text { Oligodendrocyte precursor cell } \\ \text { OTO } & \text { Osmium thiocarbohydrazide osmium } \\ \text { P } & \text { Postnatal day } \\ \text { p } & \text { P-value } \\ \text { p75NTR } & \text { Neurotrophin receptor p75 } \\ \text { pA } & \text { Picoampere } \\ \text { PB } & \text { Phosphate buffer } \\ \text { PBS } & \text { Phosphate buffered saline } \\ \text { PCR } & \text { Polymerase chain reaction } \\ \text { PFA } & \text { Paraformaldehyde } \\ \text { PH } & \text { Pleckstrin homology } \\ \text { pH } & \text { Potential hydrogen } \\ \text { PI3K } & \text { Phosphoinositide 3-kinase } \\ \text { PIP } 2 & \text { Phosphatidylinositol 4,5-bisphosphate } \\ \text { PIP } 3 & \text { Phosphatidylinositol 3,4,5-trisphosphate } \\ \text { PL } & \text { Paranodal loop } \\ \text { pmol } & \text { Picomoles } \\ \text { PNS } & \text { Peripheral nervous system } \\ \text { Ppia } & \text { Peptidylprolyl isomerase A } \\ \text { PVDF } & \text { Polyvinylidene difluoride } \\ \text { qRT-PCR } & \text { Quantitative real time PCR } \\ \text { R } & \text { Arginine } \\ \text { RIPA } & \\ \text { RNA } & \text { RNA-Seq } \\ & \end{array}$




$\begin{array}{ll}\text { rpm } & \text { Revolutions per minute } \\ \text { RT }{ }^{\circ} \mathrm{C} & \text { Room temperature } \\ \text { SDS } & \text { Sodium dodecyl sulfate } \\ \text { SDS PAGE } & \text { Sodium dodecyl sulfate-polyacrylamide gel electrophoresis } \\ \text { sec } & \text { Seconds } \\ \text { SEM } & \text { Standard error of the mean } \\ \text { SLI } & \text { Schmidt-Lanterman incisures } \\ \text { SpJ } & \text { Septate-like junction } \\ \text { SUE } & \text { Septin unique element } \\ \text { TBE } & \text { Tris-borat-EDTA } \\ \text { TBS } & \text { Tris-buffered saline } \\ \text { TBST } & \text { Tris-buffered saline with Tween-20 } \\ \text { TEMED } & \text { Tetramethylethylendiamin } \\ \text { tRNA } & \text { Transfer ribonucleic acid } \\ \text { UV } & \text { Ultraviolett } \\ \text { v/v } & \text { Volume per volume } \\ \text { V } & \text { Volt } \\ \text { w/v } & \text { Weight per volume } \\ \text { W } & \text { Tryptophan } \\ \text { Wt } & \text { Wild type } \\ \end{array}$




\section{Protein names}

\section{Abbreviation}

ACTBL2

ADAM22

ANLN

APP

ARPC2

ATP1A1

ATP1A3

CA2

CADM4

Cas9

CASPR

CASPR2

CDC42

CLD11

CNP

CNTN

CNTN2

COR01C

EPB41L3

GFAP

GLUT1

GSN

IBA1

$\mathrm{K}_{\mathrm{v}} 1.1$

$\mathrm{K}_{\mathrm{v}} 1.2$

$\mathrm{Kv} \beta 2$

L-MAG

MAG

MAC3

\section{Protein name}

Actin beta-like 2

Metalloproteinase 22

Anillin

Amyloid precursor protein

Actin-related protein 2/3 complex subunit 2

ATPase $\mathrm{Na}+\mathrm{K}+$ transporting subunit alpha 1

ATPase $\mathrm{Na}+\mathrm{K}+$ transporting subunit alpha 3

Carbonic anhydrase II

Cell adhesion molecule 4

CRISPR associated protein 9

Contactin associated protein 1

Contactin associated protein 2

Cell division control protein 42 homolog

Claudin 11

2'3'-cyclic nucleotide 3'phosphodiesterase

Contactin 1

Contactin 2

Coronin-1C

Erythrocyte membrane protein band 4.1 like 3

Glial fibrillary acidic protein

Glucose transporter 1

Gelsolin

Ionized calcium-binding adapter molecule 1

Potassium voltage-gated channel subfamily A member 1

Potassium voltage-gated channel subfamily A member 2

Voltage-gated potassium channel beta 2 subunit

Large myelin-associated glycoprotein

Myelin associated glycoprotein

Lysosomal-associated membrane protein 2 (LAMP2/MAC3) 
MAP1A

MAP1B

MAP1LC3A

MAP6

MAPT

MBP

MOBP

MOG

$\mathrm{Na}_{\mathrm{v}} 1.1$

$\mathrm{Na}_{\mathrm{v}} 1.2$

$\mathrm{Na}_{v} 1.6$

$\mathrm{Na}_{v} 1.8$

$\mathrm{Na}_{v} 1.9$

NF

NFASC

NFASC155

OMG

PLLP

PLP

PTEN

RDX

$\mathrm{RHOA}$

$\mathrm{RHOB}$

SEPT2

SEPT4

SEPT7

SEPT8

SEPT9

SEPT11

SIRT2

TLR4

TNF
Microtubule associated protein 1A

Microtubule associated protein 1B

Microtubule associated protein 1 light chain 3 alpha

Microtubule associated protein 6

Microtubule associated protein tau

Myelin basic protein

Myelin-associated oligodendrocytic basic protein

Myelin oligodendrocyte glycoprotein

Sodium channel, voltage-gated, type I, alpha subunit

Sodium channel, voltage-gated, type II, alpha subunit

Sodium channel, voltage gated, type VIII, alpha subunit

Sodium channel, voltage gated, type $\mathrm{X}$, alpha subunit

Sodium channel, voltage gated, type XI, alpha subunit

Neurofilament

Neurofascin

Neurofascin-155

Oligodendrocyte-myelin glycoprotein

Plasmolipin

Proteolipid protein

Phosphatase and tensin homolog

Radixin

Ras homolog gene family, member $\mathrm{A}$

Ras homolog gene family, member B

Septin 2

Septin 4

Septin 7

Septin 8

Septin 9

Septin 11

Sirtuin 2

Toll-like receptor 4

Tumor necrosis factor 
TPPP

TPPP3

TUBA4A

TUBB3

TUBB4A

TUBB4B

TUBB5

TUJ1
Tubulin polymerization-promoting protein

Tubulin polymerization-promoting protein family member 3

Tubulin alpha-4A

Tubulin beta-3

Tubulin beta-4A

Tubulin beta-4B

Tubulin beta-5

Class III beta-tubulin 


\section{Abstract}

Myelination of axons facilitates rapid and precise impulse propagation in the nervous system and thus, normal motor, sensory, and cognitive capabilities. In the central nervous system (CNS), myelin is formed by oligodendrocytes, which enwrap axons with several layers of compacted membrane. The axon/myelin-unit is often impaired in myelin-related disorders and upon normal aging. However, only little is known about proteins that maintain the structure of mature myelin, many of them localized to its non-compacted subcompartments. For example, one of the latest steps of myelin maturation in the CNS is the assembly of septin filaments, which prevent the formation of pathological myelin outfoldings. This work focusses on the analysis of $A n I n^{f / f l} ; C_{n} p^{C r e / W t}(A n l n \mathrm{CKO})$ mice, which lack the cytoskeletal adaptor protein anillin (ANLN) in myelinating oligodendrocytes. To assess the role of ANLN for myelination, different biochemical and microscopic analyses have been performed. By quantitative mass spectrometry (MS) analysis of purified myelin and immunoblot validation, a specific reduction of ANLN, SEPT2, SEPT4, SEPT7, and SEPT8 has been detected in Anln cKO mice compared to controls. Electron micrograph analysis revealed the emergence of pathological myelin outfoldings upon conditional ANLN deletion and the observed reduction of myelin septins. Interestingly, these myelin outfoldings are a very specific neuropathology, as other analyzed neuropathological features were not observed in Anln cKO mice. By focused ion beam-scanning electron microscopy (FIBSEM), it has been observed that these outfoldings are large sheets of multiple compact membrane layers, a plausible explanation for an altered membrane resistance along myelinated fiber tracts and thus, the observed reduced nerve conduction velocity. The results lead to the assumption that anillin-dependent scaffolding of mature myelin by septin filaments is required for its normal function in facilitating rapid nerve conduction in the healthy CNS. 


\section{Introduction}

\subsection{The central nervous system}

The central nervous system (CNS) is classified into the brain and the spinal cord. It is essential for sensory and motor functions, which are rendered possible by signal propagation along axons. To allow fast signal propagation, evolution came up with two different strategies (Figure 1) (Rushton, 1951). First, increasing axon diameter like giant axons in some Bilateria. These giant axons are capturing a lot of space, which compete with complexity in a restricted space. To overcome this spatial problem, vertebrates secondly have evolved specialized cells that enwrap axons and thereby insulating them. The latter form of increasing nerve conduction of action potentials along the axons allows faster signal propagation, even though the axon diameter remains small (Alberts, 2002). These specialized cells are oligodendrocytes in the CNS, while in the peripheral nervous system (PNS) Schwann cells enwrap axons. Beside neurons and oligodendrocytes, the CNS consists of astrocytes and microglia, whereby oligodendrocytes, astrocytes and microglia are classified into glia cells (Kettenmann and Ransom, 2005).

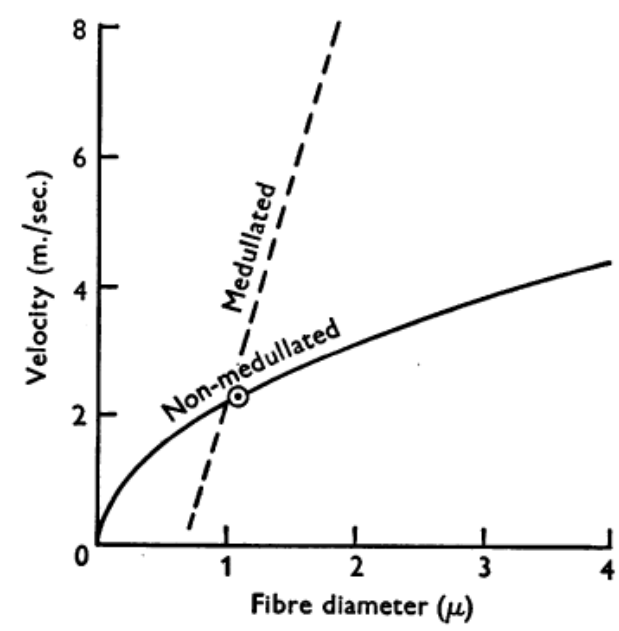

Figure 1: Theoretical relation between conduction velocity and axon diameter. Dashed line indicates the relation between conduction velocity and axon diameter for myelinated axons. The permanent line indicates the theoretical relation for non-myelinated axons. The point marks the largest c-fibers (Figure 5 from Rushton, 1951).

\subsubsection{Oligodendrocytes}

As described above, oligodendrocytes wrap their membrane around axons and thereby enable fast signal propagation. Oligodendrocytes are multipolar and build up multiple processes to form several myelin sheaths (Figure 3A). Between myelin sheaths, short nonmyelinated segments are present, the so-called nodes of Ranvier. Myelin restricts action potentials to these non-myelinated segments and thereby provides the basis for saltatory nerve conduction (Baumann and Pham-Dinh, 2001, Kettenmann and Ransom, 2005). Myelinating glia cells constitute different compartments by enwrapping axons. The 
innermost (adaxonal) and outermost (abaxonal) layer, as well as incisures and paranodal loops are classified as non-compact myelin, and are comprised of cytoplasm and cytoskeletal filaments (Arroyo and Scherer, 2000). The compact myelin is formed by multiple adhesive membrane layers (Figure 2).

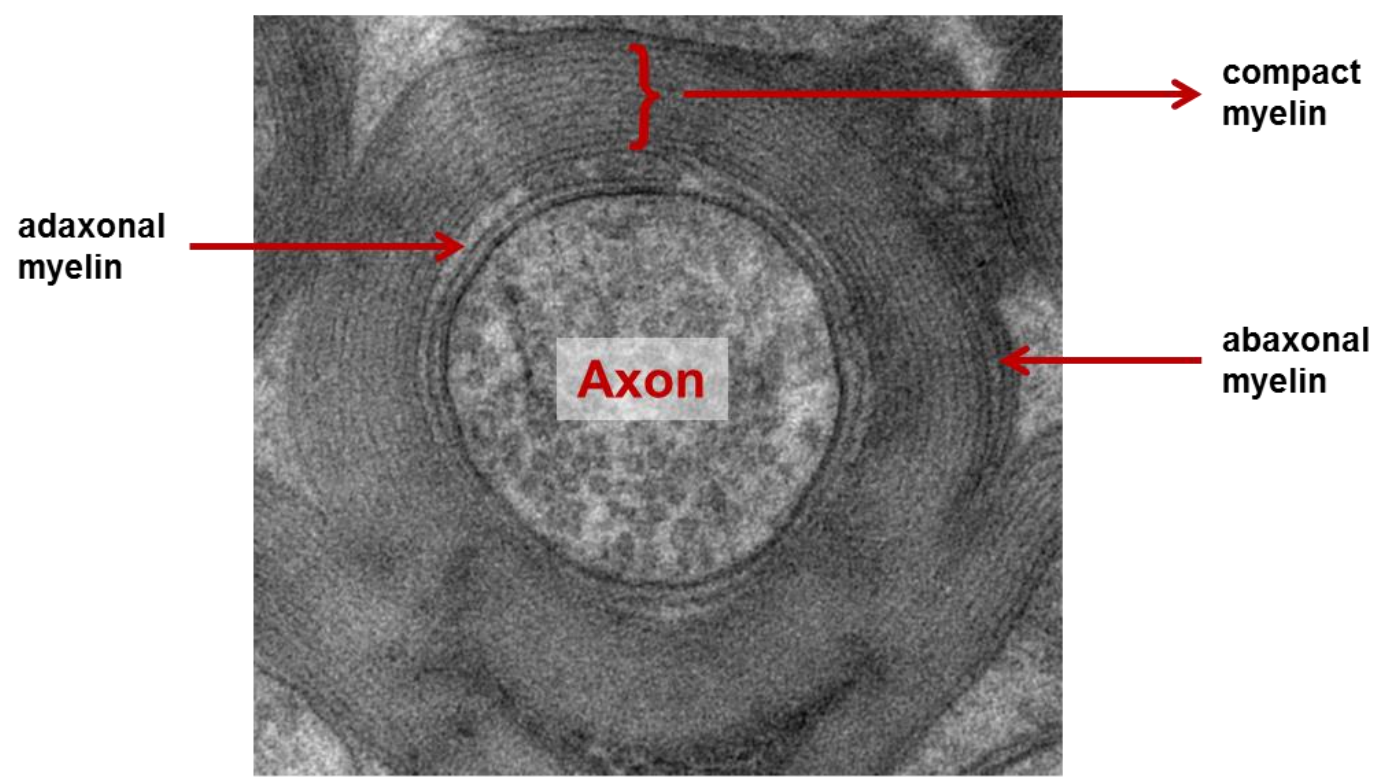

Figure 2: Illustration of compact and non-compact myelin in optic nerve cross section. Electron micrograph of a high pressure frozen optic nerve cross section, illustrates an axon and the corresponding myelin layers. Adaxonal and abaxonal myelin represent non-compact myelin. Densely packed myelin layers represent compact myelin.

The myelin sheath also leads to segmentation along the axon (Figure 3B). Each segment has a specific function and molecular composition. The node of Ranvier is a non-myelinated segment where voltage-gated sodium channels ( $\left.\mathrm{Na}_{v} 1.1, \mathrm{Na}_{v} 1.2, \mathrm{Na}_{v} 1.6, \mathrm{Na}_{v} 1.8, \mathrm{Na}_{v} 1.9\right)$ are clustered (Bennett, 2013, Salzer, 2003, Zhou et al., 1998). Furthermore, subtypes of potassium channels, axonal transmembrane proteins, and axonal cytoskeletal components are found (Bennett, 2013, Berghs et al., 2000, Sherman et al., 2005). The nodes of Ranvier are flanked by paranodes. In the paranodal segment, the paranodal loops of the enwrapping oligodendrocytes are in close contact to the axon via septate-like junctions (SpJ). These junctions are build up by adhesion proteins located in the axon (contactin-associated protein (CASPR), contactin (CNTN)) and in the myelin membrane (neurofascin-155 (NFASC155)) (Einheber et al., 1997, Salzer et al., 2008). The adhesion complex is anchored to the axonal cytoskeleton via the erythrocyte membrane protein band 4.1 like 3 (EPB41L3) (Boyle et al., 2001, Gollan et al., 2002). Beside the paranode, the juxtaparanode (JXP) is located. Here, voltage-gated potassium channel subunits $\left(\mathrm{K}_{\mathrm{v}} 1.1, \mathrm{~K}_{\mathrm{v}} 1.2\right)$ as well as a cytoplasmic subunit $(\mathrm{Kv} \beta 2)$ are located (Rasband, 2011). The clustering of these potassium channel subunits is regulated by CASPR2 and CNTN2 (Poliak and Peles, 2003). Moreover, the complex consists of disintegrin and metalloproteinase 22 (ADAM22) (Ogawa et al., 2010). The last 
segment is the internode, which is tightly enwrapped with myelin, facing the adaxonal myelin membrane.

A

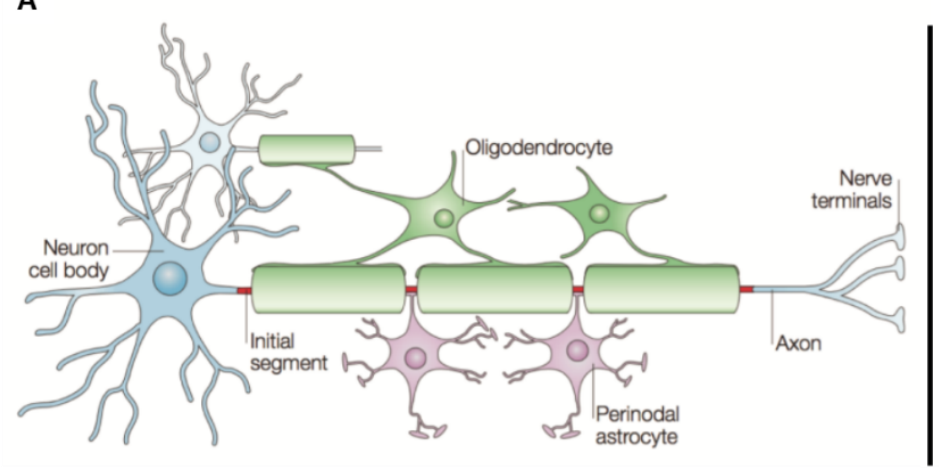

B

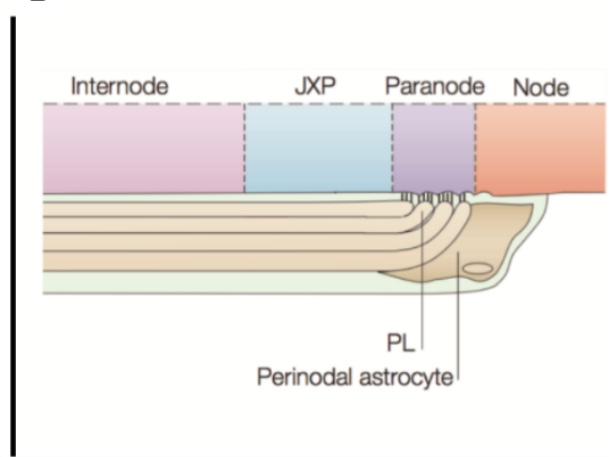

Figure 3: Structure of CNS myelinated axons. (A) Scheme of myelination in the central nervous system. Oligodendrocytes form multiple myelin sheaths and thereby enable salutatory signal propagation. (B) Scheme of the segmentation of a heminode. By myelinating an axon, the oligodendrocyte segments the axon into different parts. The node is not covered by myelin, whereas at the paranode the paranodal loops $(\mathrm{PL})$ are in close contact with the axon via septate-like junctions. The juxtaparanode (JXP) is classified by a high abundance of potassium channels, whereas the internode is the largest segment covered by adaxonal myelin (modified Figure 1 from Poliak and Peles, 2003).

For a long time, it was thought that the only role of oligodendrocytes is the insulation of an axon and the accompanied saltatory nerve conduction. Nowadays, there is more and more evidence that oligodendrocytes are important for the axonal integrity by providing trophic support (Nave, 2010, Funfschilling et al., 2012, Lee et al., 2012).

\subsubsection{Astrocytes}

Astrocytes are the most abundant cells in the CNS and have several important functions, e.g. supplying neurons with oxygen and nutrients, store nutrients (glycogen), transport neuronal metabolites, such as $\mathrm{CO}_{2}$, and take up neurotransmitters from the synaptic cleft (Fields and Stevens-Graham, 2002). They are indispensable for functional signal propagation, as they terminate neurotransmission and contribute to the ion homeostasis. In addition, astrocytes are interconnected via gap junctions and can communicate with each other over long distances (Kirchhoff et al., 2001). Furthermore, astrocytes are in direct contact with axons at the nodes of Ranvier and thought to have a stabilization and supply function for the nodes (perinodal astrocyte) (Black and Waxman, 1988). Astrocytes are also involved in the formation of the blood-brain barrier (BBB) by contacting blood vessels. Via the glucose transporter GLUT1, they are able to take up glucose from capillaries, and therefore are able to supply oligodendrocytes and neurons with lactate (via glycolysis) (Nave and Werner, 2014).

After CNS injury, astrocytes react to biochemical changes, leading to astrogliosis around the lesion. This defense response has the goal to minimize and repair primary damage. In 
contrast to the goal of repair, astrocytes often release high levels of molecules that suppress neuron outgrowth and thereby inhibit axon regeneration (Sharma, 2015).

\subsubsection{Microglia}

Microglia account for $\sim 10 \%$ of cells found within the brain. They are the main macrophages of the brain and therefore involved in, e.g. phagocytosing cell debris. Furthermore, they initiate immune responses upon for example lesions or infections. Microglia are highly mobile and scanning the environment constantly for any signs of disturbance. By detecting signs of disturbance, like cell debris or cell injury, microglia respond with repair or phagocytosis (Hanisch and Kettenmann, 2007, Nimmerjahn et al., 2005, Streit et al., 2005). In a disease state, like a lesion or infection, microglia can change into a different state, in which they can actively migrate towards the place of disturbance. In close proximity to this place, microglia induce specific responses, e.g. phagocytosis or cytokine and chemokine secretion upon detection of the relevant molecules with their various receptors (Kettenmann et al., 2011, Hanisch, 2002). Microglia support the axonal regeneration by phagocytosing cell debris and secondary release of TLR4 or TNF. If cell debris is not removed, which possibly leads to a chronic or false activation of microglia, axons get degenerated (Figure 4) (Rivest, 2009). 


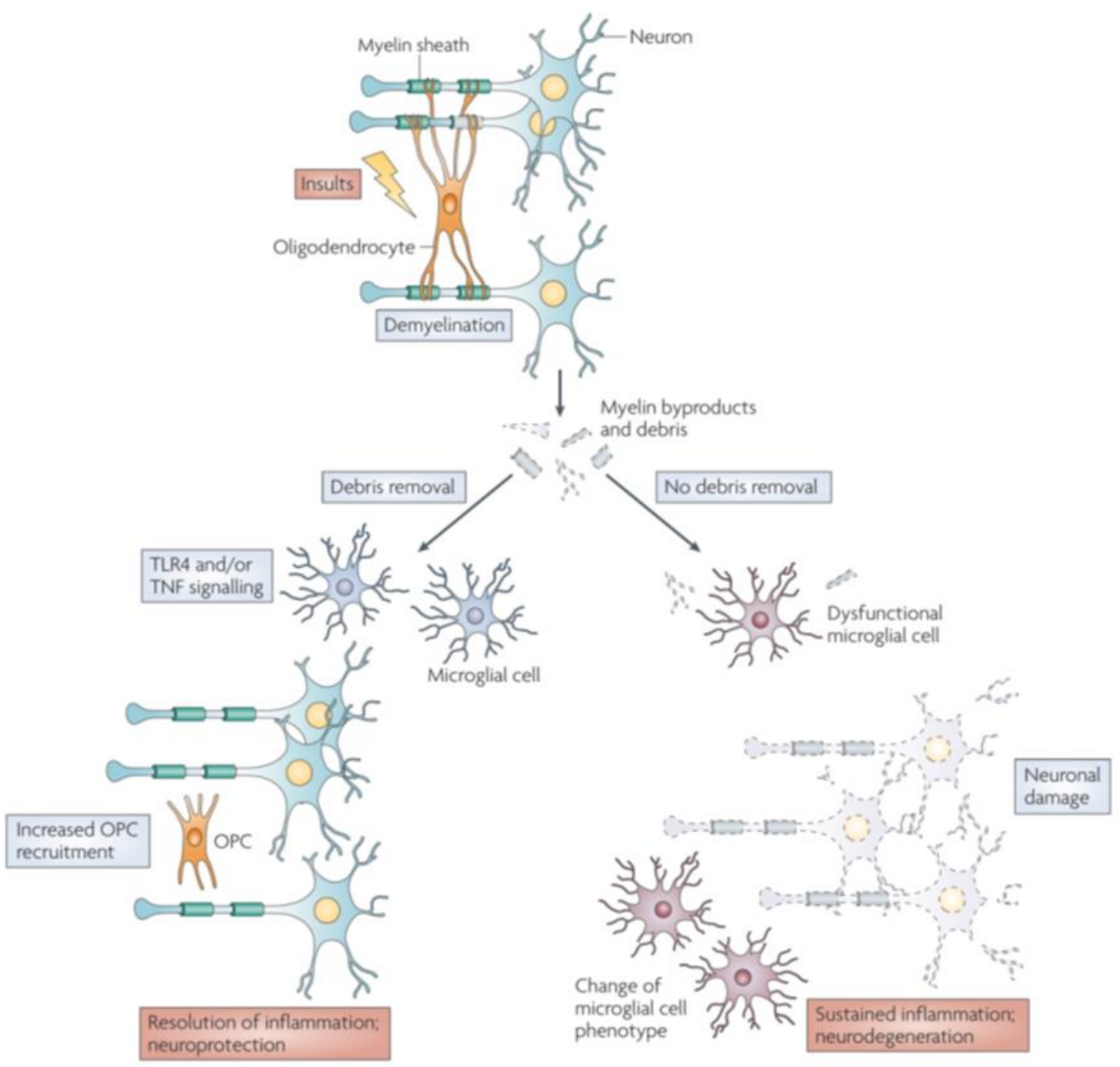

Figure 4: Neuroprotection or neurodegeneration induced by microglia. Upon demyelination, microglia are in charge of cell debris removal. By removing cell debris and releasing TLR4 or TNF, microglia act in a neuroprotective way. More oligodendrocyte precursor cells (OPCs) are recruited, which re-myelinate axons and thereby prevent axon degeneration. If microglia fail to phagocytose cell debris, neurons are damaged and further degraded, leading to neurodegeneration (Rivest, 2009) (Image taken from Rivest, 2009 (Figure 5)).

\subsection{Schwann cells}

Schwann cells are the glia cells of the peripheral nervous system (PNS). They are subdivided into myelinating Schwann cells and non-myelinating Schwann cells, which form Remak bundles (Figure 5). Schwann cells are important for the maintenance of axons and the survival of neurons. 


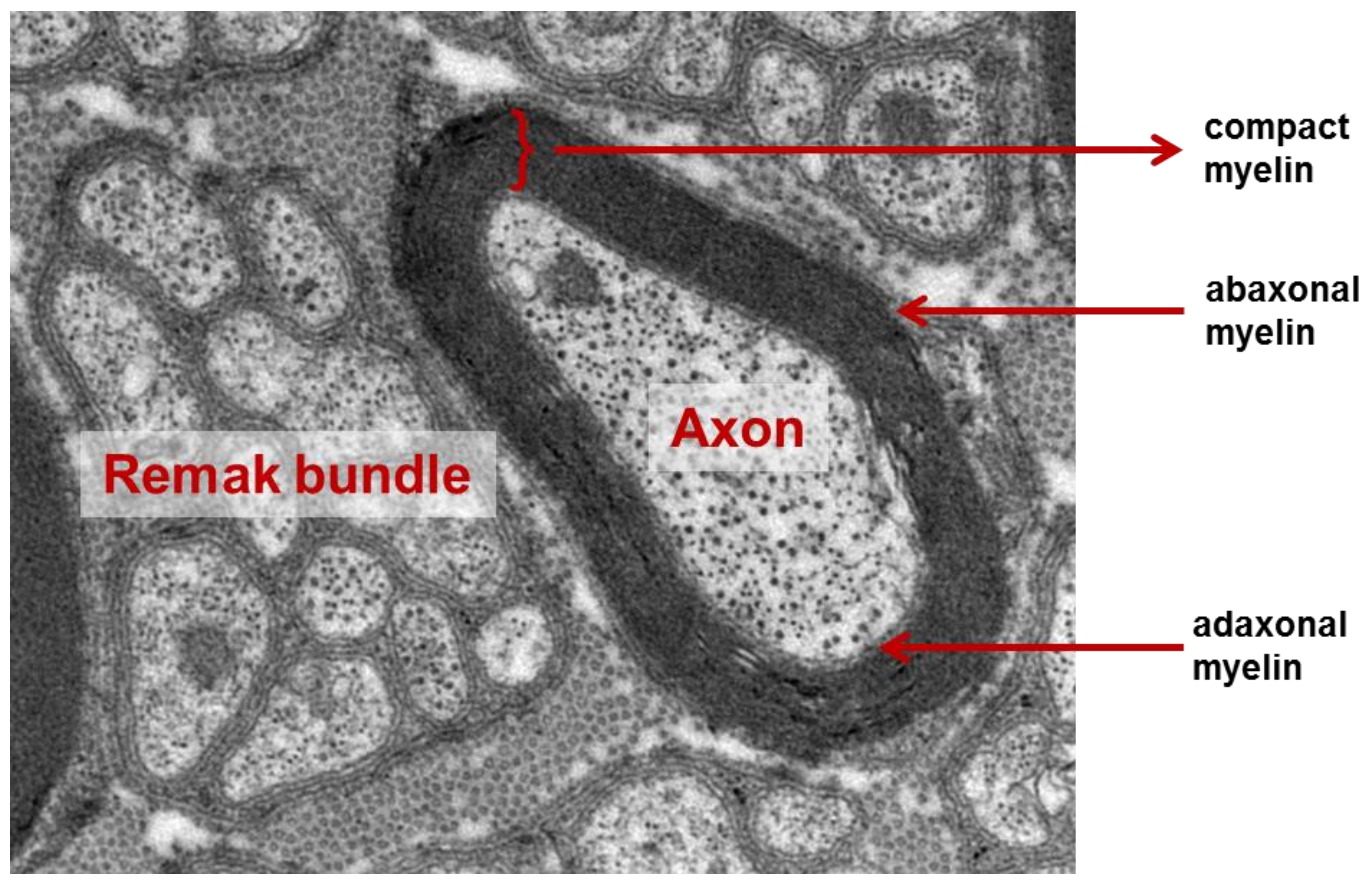

Figure 5: Schwann cell types in the PNS. Electron micrograph of a sciatic nerve cross section illustrates both types of Schwann cells. Non-myelinating Remak bundle group C fibers together and ensure that the axons are in close proximity but do not touch each other. Therefore, the cytoplasm of the non-myelinating Schwann cell surrounds the axons. Myelinating Schwann cells form myelin around the axon, exhibiting the same structures as oligodendrocytes in the CNS (compact myelin and the non-compact abaxonal and adaxonal layers).

\subsubsection{Myelinating Schwann cells}

In the PNS, myelinating Schwann cells form myelin around motor and sensory neurons, thereby insulating the axon and enable saltatory nerve conduction, like oligodendrocytes in the CNS. Different from oligodendrocytes, Schwann cells just form one myelin sheath (Figure 6A). The segmentation in node, paranode, juxtaparanode and internode is the same as in the CNS, including the clustering of ion channels. The node is partially in contact with microvilli of the Schwann cell and the myelinated fiber is surrounded by a basal lamina (Figure 6B).

A

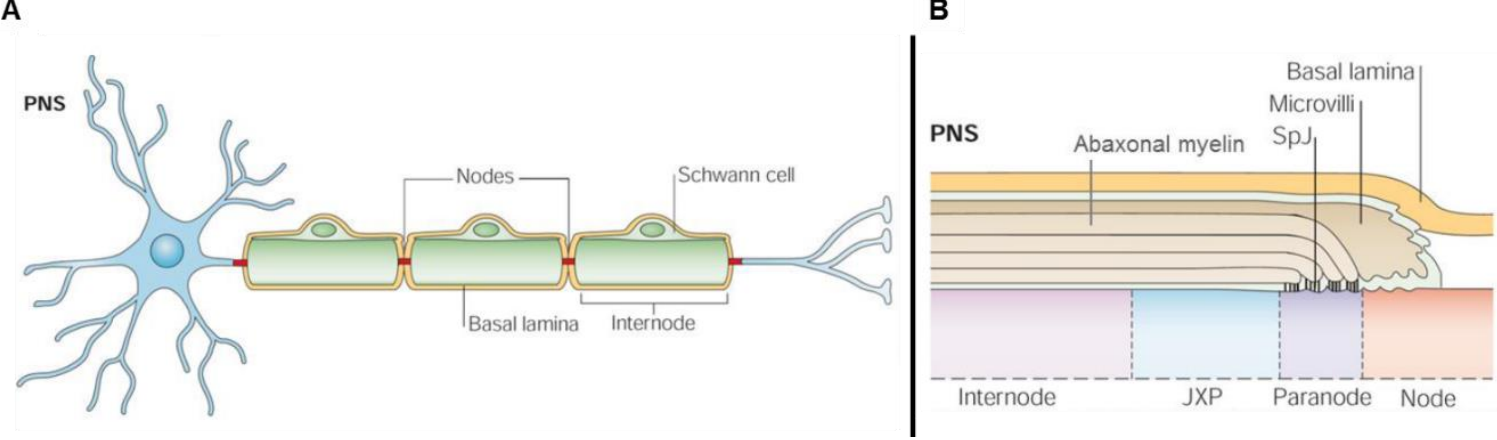

Figure 6: Structure of PNS myelinated axons. (A) Scheme of myelination in the peripheral nervous system. Each Schwann cell forms just one myelin sheath around an axon and thereby enable salutatory signal propagation. (B) Scheme of the segmentation of a heminode. By myelinating an axon, the Schwann cell segments the axon into different parts, like oligodendrocytes in the CNS. The segments are divided in node, paranode with septate-like junctions (SpJ), juxtaparanode (JXP) and the internode (modified Figure 1 from (Poliak and Peles, 2003)). 


\subsubsection{Remak bundle}

Non-myelinating Schwann cells form so-called Remak bundles. Within these Remak bundles, several small caliber axons are grouped together. These axons are $\mathrm{C}$ fibers that have a very small axon diameter $(<1 \mu \mathrm{m})$ and low conduction velocity. In a Remak bundle, axons are grouped together but are separated from each other by the cytoplasm of the nonmyelinating Schwann cell. C fibers are sensory fibers and therefore often involved in neuropathic pain.

\subsection{Myelin integrity and pathological myelin outfoldings}

As described above, oligodendrocytes enwrap axons to form myelin. It is notable, that the myelin thickness is dependent on the axon diameter, as the ratio between the axonal diameter and the myelin diameter is constantly about $0.7+/-0.1$ (Hildebrand and Hahn, 1978, Chomiak and Hu, 2009), suggesting an optimal signal propagation within this given ratio. There is a need for several signals that lead to the start of myelination, but also for attenuating myelination when the optimal myelin thickness is reached. For the PNS, it is known that axonal expressed neuregulin-1 regulates myelin thickness, whereas in the CNS, such a regulator has not been identified so far (Michailov et al., 2004, Brinkmann et al., 2008). However, there are different pathways known, which indeed influence myelin thickness and integrity. For example, mice that lack PTEN (phosphate and tensin homolog) from oligodendrocytes show increased myelin thickness (Goebbels et al., 2010). PTEN is a regulator of the mTOR pathway. By converting $\mathrm{PIP}_{3}$ (Phosphatidylinositol $(3,4,5)$ trisphosphate) into $\mathrm{PIP}_{2}$ (Phosphatidylinositol-4,5-bisphosphat), the PI3K induced mTOR pathway activation is antagonized (Stiles et al., 2004, Suzuki et al., 2008). Thereby, Akt gets over-activated and $\mathrm{PIP}_{3}$ accumulates in the plasma membrane (Cantley and Neel, 1999). Additionally, in 2016 it was shown that PTEN mutant mice develop pathological myelin outfoldings (Patzig et al., 2016). Myelin outfoldings are seen in several mouse mutants and are a common neuropathological phenotype. For example, mice that lack proteins, which are highly abundant in CNS myelin, Cnp ${ }^{\text {null/null }}, M^{\text {naul//null }}$ and Plp $1^{\text {null/null }}$ mice (Patzig et al., 2016), as well as $C d c 42^{f / f f l} ; C n p^{C r e / W t}$ mice (Thurnherr et al., 2006) show pathological myelin outfoldings. In all mutants, compact myelin is formed, whereby only Mag $^{\text {null/null }}$ and Plp1 $1^{\text {null/null }}$ mice display alterations in myelin thickness (Patzig et al., 2016, Thurnherr et al., 2006). Interestingly, these mice show reduced levels of the cytoskeletal ANLN/septin filament, indicating the relevance of cytoskeletal stabilization of CNS myelin for myelin integrity (Patzig et al., 2016). 


\subsection{Anillin}

Anillin (ANLN) is a conserved protein implicated in several cytoskeletal dynamics and cytokinesis. In 1989, ANLN was identified as a protein that binds F-actin in Drosophila melanogaster (Miller et al., 1989). It was shown that in the interphase ANLN localizes to the nucleus, while during cytokinesis it co-localizes with RhoA at the contractile ring (Field and Alberts, 1995, Piekny and Glotzer, 2008). There are further homologues of ANLN, for example, the two anillin-like proteins Boi1 $p$ and Bio2p in Saccharomyces cerevisiae. These proteins localize to the nucleus (Boi1p) and the contractile ring (Boi2p) and thereby are essential for cell growth and bud formation (Toya et al., 1999, Bender et al., 1996).

ANLN has several other cytoskeletal binding partners. Besides the binding of F-actin during cell division, anillin directly interacts with e.g. myosin, RhoA, microtubules and septins (Piekny and Glotzer, 2008, Straight et al., 2005, Piekny and Maddox, 2010, Versele and Thorner, 2005). The interaction with septins has been reported in different studies. In yeast, it was shown that ANLN and septins co-localize within the cleavage furrow and thereby enable proper cell division (Maddox et al., 2007, Oegema et al., 2000). In vitro studies have shown that anillin enhances $\mathrm{PIP}_{2}$-mediated septin filament formation (Kinoshita et al., 2002, Liu et al., 2012). The studies indicate that septin filament formation relies on anillin, at least in vitro and in yeast. Interestingly, it was shown, that anillin also relies on septins. An in vivo study shows that in CNS myelin of mice lacking septin filaments, ANLN is also reduced (Patzig et al., 2016). These results indicate that ANLN not only promotes septin filament formation but also interacts with the filament to stabilize adaxonal myelin. Brain RNA-Seq analysis of mouse cerebral cortex show expression of ANLN in oligodendrocytes (Figure 7) (Zhang et al., 2014).

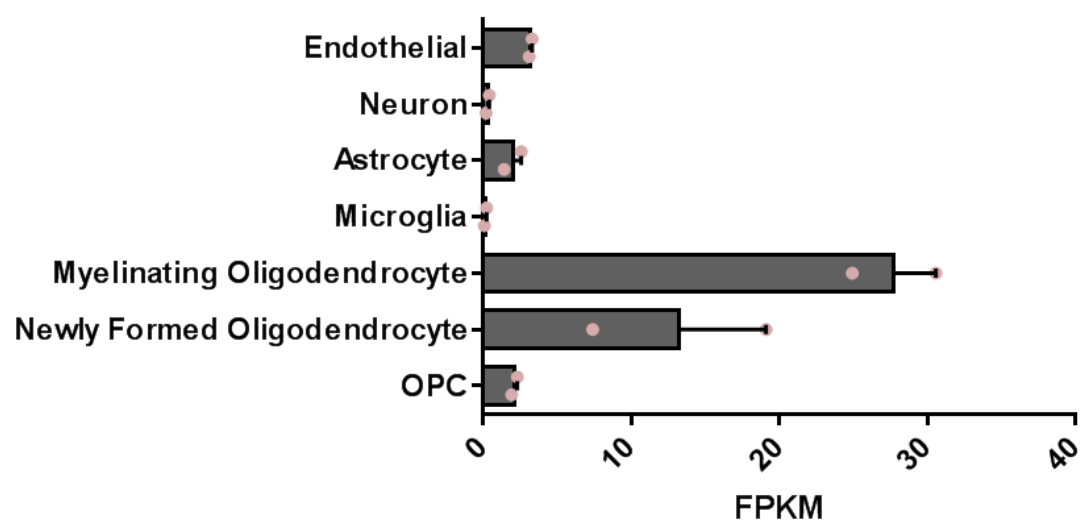

Figure 7: RNA-Seq analysis indicates AnIn mRNA expression in oligodendrocytes. RNA-Seq analysis of cells immunopanned from mouse cerebral cortex, shows expression of Anln mRNA. Anln mRNA is detected in myelinating and newly formed oligodendrocytes. The cell-type specific abundance was extracted from Zhang et al., 2014. Mean with SEM; $n=2 ; F P K M=$ fragments per kilobase of exon per million fragments mapped; OPC = oligodendrocyte precursor cell. 


\subsection{Septins}

Septins are a family of highly conserved GTP-binding proteins, containing an N-terminal polybasic domain and a septin unique element (SUE) at the C-terminal. The proline-rich $\mathrm{N}$-terminus is variable in length and the $\mathrm{C}$-terminal end can contain a coiled-coil region (Cao et al., 2007). In mammals, 13 septin genes are known. Most of them encode more than one polypeptide by alternative splicing and/or multiple translation start sites (Kinoshita, 2003). The mammalian septin gene family can be divided into four subgroups, which are named after the representing septins, by sequence comparison and bioinformatic analysis. SEPT1, SEPT2, SEPT4 and SEPT5 belong to the SEPT2 group, whereby every member shows two C-terminal coiled-coil domains. The SEPT6 group has five members; SEPT6, SEPT8, SEPT10, SEPT11 and SEPT14; all of them have one C-terminal coiled-coil domain. SEPT3 is the third group, consisting of SEPT3, SEPT9 and SEPT12; this group has no coiled-coil domain. The last group only consists of SEPT7 (Mostowy and Cossart, 2012, Hall and Russell, 2012, Macara et al., 2002). SEPT13 is not classified, as it was identified to be a pseudogene of SEPT7 (HGNC: 32339).

Septin monomers build up hetero-oligomers upon GTP binding and are able to assemble even further into higher order structures, like rings, meshwork or filaments (Mendoza et al., 2002, Oh and $\mathrm{Bi}, 2011)$. It was shown by several studies that a hetero-oligomer consists of septins out of different subgroups in a 1:1:1 ratio (Kinoshita, 2003). In an in vitro experiment, for example, a hetero-oligomer consisting of SEPT2, SEPT6, and SEPT7 assembled into filaments (Low and Macara, 2006, Sirajuddin et al., 2007). It was also shown that septins have various functions in cytokinesis, ciliogenesis, dendrite maturation, and sperm development (Hall and Russell, 2004, Kissel et al., 2005, Kwitny et al., 2010, Tada et al., 2007, Xie et al., 2007, Mostowy and Cossart, 2012). Septin oligomers polymerize further into a meshwork upon interaction with membrane lipids. This meshwork is comparable to an actin cytoskeleton, but it is less dynamic (Tooley et al., 2009). It was therefore suggested that septins play a role in stabilizing membranes (Tanaka-Takiguchi et al., 2009, Gilden and Krummel, 2010). Indeed, it was shown that septins stabilize the adaxonal myelin membrane and thereby prevent the formation of pathological myelin outfoldings in the CNS of mice (Patzig et al., 2016). The study also shows that SEPT2, SEPT4, SEPT7, and SEPT8 are the most abundant septins in CNS myelin. Furthermore, SEPT8 is essential to form a septin filament in CNS myelin, as the loss of SEPT8 leads to post-translational reduction of all other myelin septins (Patzig et al., 2016).

\subsection{Other cytoskeletal filaments}

Actin filaments are composed of globular actin (G-actin) monomers that polymerize into filamentous actin (F-actin). Within a filament, all actin monomers are orientated in the same 
direction, leading to a polar actin filament. This polarization gives raise to the plus and minus end, which on the one hand sets the direction of assembly of the filament and on the other hand enables a directed movement of myosin along the actin filament (Pollard, 2017, Oda et al., 2009). Actin filaments are often found in close proximity to plasma membranes, where the build meshwork has functions in mechanical support, cell shape determination, and enables movement of the cell surface to allow e.g. cell migration or division (Cooper, 2000). Another compartment of the cytoskeleton are intermediate filaments. There are different proteins that build up intermediate filaments cell type specific, e.g. lamin (nuclear envelope), neurofilaments ((NF) neural cells) or keratin (epithelial cells) (Dechat et al., 2010, Yuan et al., 2012, Chou and Buehler, 2012). Similar to actin filaments, intermediate filaments have functions in the maintenance of the cell shape. They anchor cell organelles and thereby keep up the 3-dimensional cell shape (Herrmann et al., 2007).

In addition, microtubules are a part of the cytoskeleton. $\alpha$ - and $\beta$-tubulin dimers build up protofilaments, which associate laterally into a microtubule. Like actin filaments, microtubules have a plus and a minus end, arising from the filament formation, as the $\beta$-subunit of the tubulin dimer always contacts the $\alpha$-subunit of the next dimer. Therefore, at the plus end the $\beta$-subunits are exposed, whereas at the minus end the $\alpha$-subunits are exposed. Microtubules can extend at both, plus and minus ends to elongate (Walker et al., 1988). Microtubules have important functions in e.g. the mitotic spindle or intracellular transport. The intracellular transport is associated with the motor proteins kinesin and dynein, which transport for example mitochondria along microtubules (Hirokawa and Takemura, 2005, Vale, 2003).

\subsection{Axon caliber}

Beside myelination, the axon diameter influences the signal propagation along axons, as the transverse resistance of an axon decreases with increasing caliber (Hartline and Colman, 2007, Seidl, 2014). For the CNS and PNS, different mechanisms, by which the axonal diameter is regulated, have been identified. For example, in the PNS it was shown that the phosphorylation state of the neurofilaments NF-medium and NF-heavy plays an important role in axonal diameter regulation (Garcia et al., 2003). Interestingly, the phosphorylation state of neurofilaments is regulated by Schwann cells in an "outside-in" fashion via the interaction of myelin associated glycoprotein (MAG) with e.g. the receptor p75NTR on the axon (de Waegh et al., 1992, Yin et al., 1998, Garcia et al., 2003, Wang et al., 2002). Furthermore, in the CNS the multimeric Nogo receptor 1 (NgR1) was identified as an axonal interaction partner of MAG in vitro using a soluble form of MAG (Palandri et al., 2015). It has been shown that mice deficient for Nogo1 $\left(\mathrm{Ngrr}^{-/}\right)$showed reduced 
neuronal somata in vitro as well as decreased axonal diameter in the optic nerve and the spinal cord in vivo (Palandri et al., 2015, Lee et al., 2017).

Furthermore, axonal intrinsic mechanisms regulating the axon diameter include $\alpha$-adducin and PTEN. It has been shown that the loss of a-adducin, an actin binding protein that stabilizes actin rings along the axon, show a progressive enlargement of axon diameter (Leite et al., 2016). Moreover, mice that conditionally lack PTEN in cerebellar granule cells, showed increased neuronal diameter of these specific neuron population in the cortex (Goebbels et al., 2017).

\subsection{Aim of the study}

Deletion of the Sept8 gene in mature oligodendrocytes prevents the assembly of myelin septin filaments; notably, when myelin septin filaments are absent the abundance of ANLN in myelin is also diminished (Patzig et al., 2016). This implies that the association with myelin septin filaments prevents rapid turnover and degradation of ANLN. Here, the hypothesis is to be tested that oligodendroglial ANLN, beyond its mere association with myelin septins, may serve a crucial function in myelin septin filament assembly. To do so, a novel line of $A n l n^{f / f t l} ; C n p^{C r e / W t}(A n l n$ cKO) mouse mutants is to be analyzed, in which mature oligodendrocytes do not express ANLN. The loss of ANLN and its effect on myelin septin assembly, as well as related myelin pathology is to be analyzed. For understanding the possible relevance of ANLN for septin filament assembly in CNS myelin, immunohistochemistry is to be used to test for co-localization of ANLN and septins in Wt mice. Additionally, it is to be tested if a developmental dependence of ANLN and septins can be identify. To test the functional relevance of ANLN for myelin septins, ANLN is to be deleted in mature oligodendrocytes of mice, using Cre recombinase under control of the Cnp promotor (Cnp ${ }^{\text {Cre/Wt }}$ (Lappe-Siefke et al., 2003)). The abundance of ANLN and septins is to be analyzed using quantitative mass spectrometry, as well as immunoblot validation. Thereby, an unbiased identification of other effected proteins is possible. As the loss of septins is directly related to structural changes in myelin, possible myelin abnormalities upon the absence of ANLN, are to be analyzed. Furthermore, axonal changes upon ANLNabsence are to be analyzed. As oligodendrocytes are reported to support axonal integrity (Klugmann et al., 1997, Griffiths et al., 1998), secondary pathologies like axonopathy and gliosis are to be analyzed in $A n / n^{f / f t l} ; C n p^{C r e / W t}$ mouse brains by immunohistochemistry. It is of interest, to 3D reconstruct possible myelin abnormalities, such as myelin outfoldings, using focused ion beam scanning electron microscopy (FIB SEM), to get a better understanding of the size and dimension of such abnormalities. 


\section{Materials and Methods}

\subsection{Materials}

\subsubsection{General material}

General laboratory materials were purchased from BD Falcon (Heidelberg, Germany), BioRad (Munich, Germany), Gilson (Limburg-Offheim, Germany), Brand (Radebeul, Germany), Sarstedt (Nümbrecht, Germany) and Eppendorf (Hamburg, Germany). All chemicals used, were obtained from Sigma-Aldrich $\mathrm{GmbH}$ (Munich, Germany), Merck KGaA (Darmstadt, Germany) and SERVA (Heidelberg, Germany).

\subsubsection{Kits}

All kits were used according to the manufacturer instructions, unless otherwise stated.

\begin{tabular}{ll} 
Description & Manufacturer \\
\hline RNA purification 'RNeasy mini prep' & Qiagen (Portland, USA) \\
\hline DC Protein Assay (Lowry) & Bio-Rad (Munich, Germany) \\
\hline DAB Zytomed Kit & Zytomed Systems GmbH (Berlin, Germany) \\
\hline LSAB2 Kit & Dako (Hamburg, Germany) \\
\hline Vector Elite ABC Kit & Vector Labs (Loerrach, Germany) \\
\hline Agilent RNA 6000 Nano KIT & $\begin{array}{l}\text { Agilent Technologies (Santa Clara, California, } \\
\text { United States) }\end{array}$
\end{tabular}

\subsubsection{Materials for genotyping}

\begin{tabular}{llr} 
Description & Content \\
\hline Modified Gitschier buffer (MGB) & Tris/HCl, pH 8.8 & \\
& $\left(\mathrm{NH}_{4}\right)_{2} \mathrm{SO}_{4}$ & $67 \mathrm{mM}$ \\
& $\mathrm{MgCl}_{2}$ & $16.6 \mathrm{mM}$ \\
& Triton X-100 & $6.5 \mathrm{mM}$ \\
\hline Tris-borat-EDTA (TBE) buffer & Tris Base & $0.5 \%[\mathrm{v} / \mathrm{v}]$ \\
\hline & Boric acid & $40 \mathrm{mM}$ \\
& EDTA & $20 \mathrm{mM}$ \\
& & $1 \mathrm{mM}$
\end{tabular}


$10 \mathrm{mM}$ dNTP (50x stock)

$\begin{array}{ll}\text { dATP } & 2.5 \mathrm{mM} \\ \text { dCTP } & 2.5 \mathrm{mM} \\ \text { cGTP } & 2.5 \mathrm{mM} \\ \text { cTTP } & 2.5 \mathrm{mM}\end{array}$

$200 \mu \mathrm{M}$ final concentration in a PCR reaction

(50 $\mu \mathrm{M}$ each nucleotide)

\begin{tabular}{ll} 
Description & Manufacturer \\
\hline Proteinase K & Boehringer GmbH (Mannheim, Germany) \\
\hline GoTaq DNA polymerase & Promega (Mannheim, Germany) \\
\hline GoTaq buffer 5x & Promega (Mannheim, Germany) \\
\hline Superscript III-reverse transcriptase & Invitrogen (Karlsruhe, Germany) \\
\hline dNTPs & Boehringer GmbH (Mannheim, Germany) \\
\hline GeneRuler 100 bp DNA ladder & Thermo Fisher Scientific (St. Leon-Rot, Germany)
\end{tabular}

\subsubsection{Genotyping primers}

\begin{tabular}{lrl} 
Description & Number & Sequence \\
\hline \multirow{2}{*}{ Anln genotyping } & 29823 & 5'- GACATAGCCCTCAGTGTTCAGG -3' \\
29824 & 5'- GAATCCTGCATGGACAGACAG -3' \\
32002 & 5'- GAGCTCAGACCATAACTTCG -3' \\
\hline \multirow{2}{*}{ Sept8 genotyping } & 16458 & 5'- CAGGCAGATGTATATGCAGCAG -3' \\
& 16459 & 5'- GGTGGCTTTGACTTGCTATCC -3' \\
& 16460 & 5'- GAAGCAGCCATAGAGGAGATCC -3' \\
Cre recombinase & 2016 & 5'- GCCTTCAAACTGTCCATCTC -3' \\
genotyping & 7315 & 5'- CCCAGCCCTTTTATTACCAC -3' \\
& 4192 & 5'- CAGGGTGTTATAAGCAATCCC -3' \\
4193 & 5'- CCTGGAAATGCTTCTGTCCG -3' \\
\hline \multirow{2}{*}{ Mbp genotyping } & 30833 & 5'- GAGCTCTGGTCTTTTCTTGCAG -3' \\
30834 & 5'- CCCGTGGTAGGAATATTACATTAC -3' \\
30835 & 5'- CAGGGGATGGGGAGTCAG -3' \\
1879 & 5'- ATGTATGTGTGTGTGTGCTTATCTAGTGTA -3'
\end{tabular}




\begin{tabular}{lll} 
Sept9 genotyping & 23844 & 5'- GACCATGCATGCACTCAGCCACAG -3' \\
23845 & 5'- CAGAACTGAAATCCCCTACAGTAG -3' \\
23846 & 5'- GCTCAGACAACGATATTTGGG -3' \\
\hline Dhh Cre recombinase & 10967 & 5'- CCTGCGGAGATGCCCAATTG -3' \\
genotyping & 15793 & 5'- CAGCCCGGACCGACGATGAA -3'
\end{tabular}

\subsubsection{Quantitative real-time PCR primers}

\begin{tabular}{|c|c|c|}
\hline Gene & Direction & Sequence \\
\hline \multirow[t]{2}{*}{ Anln } & Forward & 5'- ACAATCCAAG GACAAACTTGC -3' \\
\hline & Reverse & 5'- GCGTTCCAGG AAAGGCTTA -3' \\
\hline \multirow[t]{2}{*}{ Cnp } & Forward & 5'- CGCTGGGGCAGAAGAATA -3' \\
\hline & Reverse & 5'- AAGGCCTTGCCATACGATCT -3’ \\
\hline \multirow[t]{2}{*}{ Sept2 } & Forward & 5'- TCCTGACTGA TCTCTACCCAGAA -3' \\
\hline & Reverse & 5'- AAGCCTCTAT CTGGACAGTTCTTT -3' \\
\hline \multirow[t]{2}{*}{ Sept4 } & Forward & 5'- ACTGACTTGT ACCGGGATCG -3' \\
\hline & Reverse & 5'- TCTCCACGGT TTGCATGAT -3' \\
\hline \multirow[t]{2}{*}{ Sept7 } & Forward & 5'- AGAGGAAGGC AGTATCCTTGG -3’ \\
\hline & Reverse & 5'- TTTCAAGTCC TGCATATGTGTTC -3' \\
\hline \multirow[t]{2}{*}{ Sept8 } & Forward & 5‘- CTGAGCCCCG GAGCCTGT -3’ \\
\hline & Reverse & 5'- CAATCCCAGT TTCGCCCACA -3' \\
\hline \multirow[t]{2}{*}{ Sept9 } & Forward & 5'- GACTCCATCCTGGAGCAGAT -3' \\
\hline & Reverse & 5'- TTTGGACTTGAAGAGGGTATTGA -3' \\
\hline \multirow[t]{2}{*}{ Sept11 } & Forward & 5'- GCTATGAACTCCAGGAAAGCA -3' \\
\hline & Reverse & 5'- GGGCGTCAATGTATTCCACT -3' \\
\hline \multirow[t]{2}{*}{ Cdc42 } & Forward & 5'- GCTGTCAAGTATGTGGAGTGCT -3' \\
\hline & Reverse & 5'- GGCTCTTCTTCGGTTCTGG -3' \\
\hline \multirow[t]{2}{*}{$R h o B$} & Forward & 5'- CAGACTGCCTGACATCTGCT -3' \\
\hline & Reverse & 5'- GTGCCCACGCTAATTCTCAG -3' \\
\hline \multirow[t]{2}{*}{ Ppia } & Forward & 5'- CACAAACGGT TCCCAGTTTT -3' \\
\hline & Reverse & 5'- TTCCCAAAGA CCACATGCTT -3' \\
\hline
\end{tabular}




\subsubsection{Protein biochemistry}

Description Content

\begin{tabular}{llr} 
10x Phosphate-buffered saline (PBS) & $\mathrm{NaCl}$ & $1.7 \mathrm{M}$ \\
& $\mathrm{KCl}$ & $34 \mathrm{mM}$ \\
& $\mathrm{Na}_{2} \mathrm{HPO}_{4} \times 2 \mathrm{H}_{2} \mathrm{O}$ & $40 \mathrm{mM}$ \\
& $\mathrm{K}_{2} \mathrm{HPO}_{4}$ & $\mathrm{pH} 7.2 \mathrm{mM}$ \\
& & \\
& & $200 \mathrm{mM}$ \\
10x Tris-buffered saline (TBS) & Tris/ $/ \mathrm{NCl}, \mathrm{pH} 7.4$ & $1370 \mathrm{M}$ \\
(for myelin purification) & $\mathrm{NaCl}$ & $1 \mathrm{x}$ \\
\hline \multirow{2}{*}{ Modified RIPA buffer } & TBS (10x for myelin purification) & $1 \mathrm{mM}$ \\
& EDTA & $0.5 \%[\mathrm{w} / \mathrm{v}]$ \\
& Sodium deoxycholate & $1.0 \%[\mathrm{v} / \mathrm{v}]$ \\
& Triton X-100 & 1 tablet $/ 10 \mathrm{ml}$
\end{tabular}

Description

Complete Mini protease inhibitor

\section{Manufacturer}

\subsubsection{SDS PAGE and immunoblot}

\begin{tabular}{|c|c|c|}
\hline Description & Content & \\
\hline \multirow[t]{5}{*}{ SDS separating gel } & Acrylamid/Bisacrylamid 29:1 & $15 \%, 12 \%$ or $10 \%[\mathrm{v} / \mathrm{v}]$ \\
\hline & Tris/HCl pH 8.8 & $0.4 \mathrm{M}$ \\
\hline & SDS & $0.1 \%[\mathrm{w} / \mathrm{v}]$ \\
\hline & APS & $0.03 \%[\mathrm{w} / \mathrm{v}]$ \\
\hline & TEMED & $0.08 \%[v / v]$ \\
\hline \multirow[t]{4}{*}{ SDS stacking gel } & Acrylamid/Bisacrylamid 29:1 & $4 \%[v / v]$ \\
\hline & Tris/HCl pH 8.8 & $125 \mathrm{mM}$ \\
\hline & APS & $0.05 \%[\mathrm{w} / \mathrm{v}]$ \\
\hline & TEMED & $0.1 \%[\mathrm{v} / \mathrm{v}]$ \\
\hline \multirow[t]{4}{*}{ 4x SDS sample buffer } & Glycerol & $40 \%[v / v]$ \\
\hline & Tris/HCl pH 6.8 & $240 \mathrm{mM}$ \\
\hline & SDS & $8 \%[w / v]$ \\
\hline & Bromphenol blue & $0.04 \%[\mathrm{w} / \mathrm{v}]$ \\
\hline
\end{tabular}




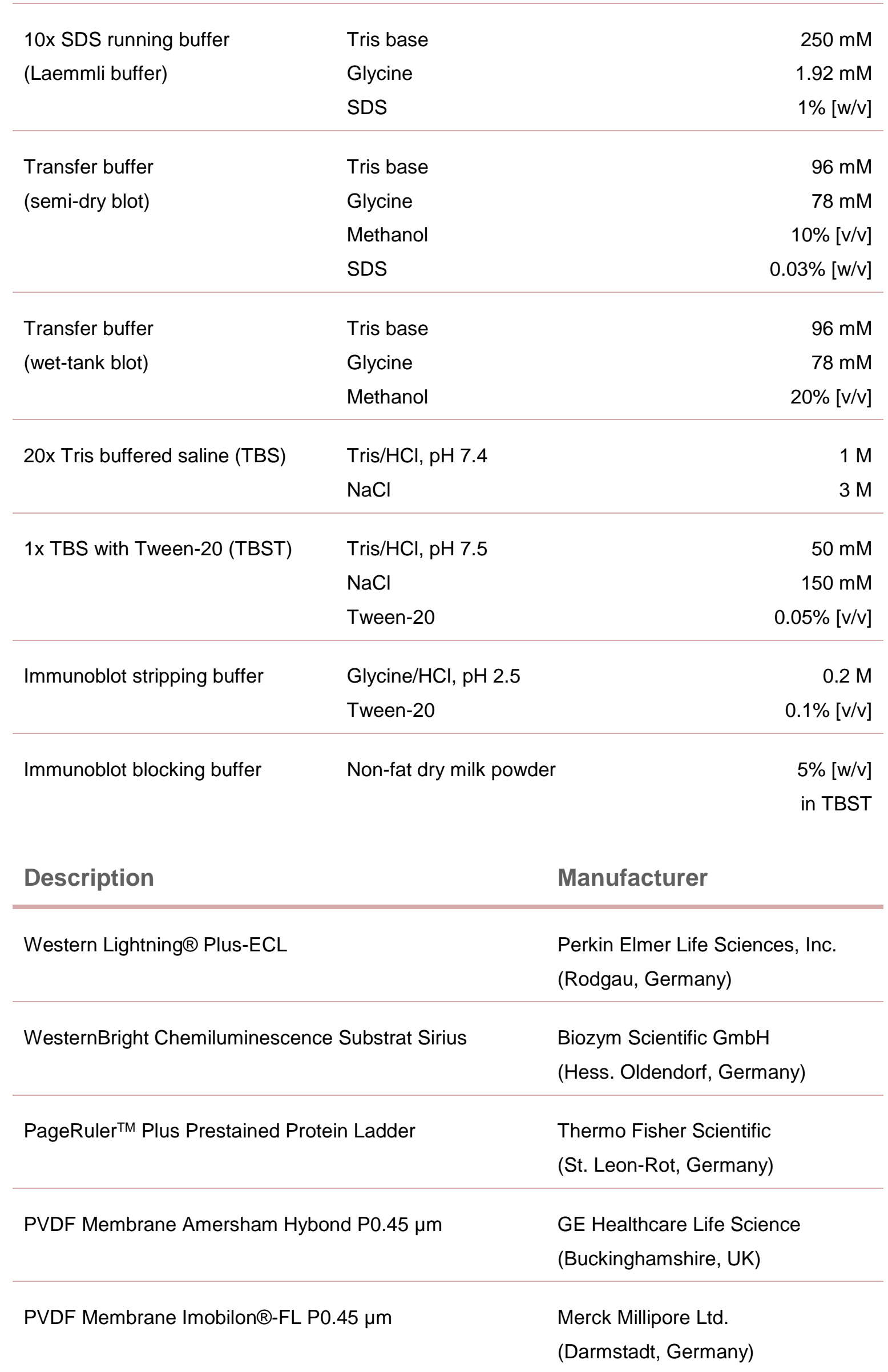




\subsubsection{Primary antibodies - Immunoblot}

\begin{tabular}{|c|c|c|c|c|c|}
\hline Antibody & Dilution & Load & $\%$ Gel & Species & Source \\
\hline ANLN & $1: 500$ & $30 \mu \mathrm{g}$ & $10 \%$ & $\begin{array}{r}\text { Polyclonal } \\
\text { goat }\end{array}$ & Acris \\
\hline SEPT2 & $1: 2000$ & $5 \mu \mathrm{g}$ & 10 or $12 \%$ & $\begin{array}{r}\text { Polyclonal } \\
\text { rabbit }\end{array}$ & $\mathrm{PtG}$ \\
\hline SEPT4 & $1: 100$ & $30 \mu \mathrm{g}$ & 10 or $12 \%$ & $\begin{array}{r}\text { Polyclonal } \\
\text { rabbit }\end{array}$ & IBL \\
\hline SEPT7 & $1: 5000$ & $3 \mu \mathrm{g}$ & 10 or $12 \%$ & $\begin{array}{r}\text { Polyclonal } \\
\text { rabbit }\end{array}$ & IBL \\
\hline SEPT8 & $1: 2500$ & $5 \mu \mathrm{g}$ & 10 or $12 \%$ & $\begin{array}{r}\text { Polyclonal } \\
\text { rabbit }\end{array}$ & $\mathrm{PtG}$ \\
\hline SEPT9 & $1: 1000$ & $30 \mu \mathrm{g}$ & 10 or $12 \%$ & $\begin{array}{r}\text { Polyclonal } \\
\text { rabbit }\end{array}$ & $\mathrm{PtG}$ \\
\hline SEPT11 & $1: 5000$ & $5 \mu \mathrm{g}$ & 10 or $12 \%$ & $\begin{array}{r}\text { Polyclonal } \\
\text { rabbit }\end{array}$ & $\begin{array}{r}\text { W.S.Trimble } \\
\text { (Tsang et al., 2011) }\end{array}$ \\
\hline L-MAG & $1: 500$ & $5-10 \mu \mathrm{g}$ & $8 \%$ & $\begin{array}{r}\text { Polyclonal } \\
\text { rabbit }\end{array}$ & $\begin{array}{l}\text { N. Schaeren-Wiemers } \\
\text { (Miescher et al., 1997) }\end{array}$ \\
\hline MOG & $1: 500$ & $5 \mu \mathrm{g}$ & $12 \%$ & $\begin{array}{r}\text { Monoclonal } \\
\text { mouse }\end{array}$ & $\begin{array}{r}\text { C. Linington (Linnington } \\
\text { et al., 1984) }\end{array}$ \\
\hline TUJ1 & $1: 1000$ & $5-10 \mu \mathrm{g}$ & 10 or $12 \%$ & $\begin{array}{r}\text { Monoclonal } \\
\text { mouse }\end{array}$ & Covance \\
\hline ATP1A1 & $1: 2000$ & $5 \mu \mathrm{g}$ & 10 or $12 \%$ & $\begin{array}{r}\text { Monoclonal } \\
\text { mouse }\end{array}$ & Abcam \\
\hline ATP1A3 & $1: 1000$ & $5 \mu \mathrm{g}$ & 10 or $12 \%$ & $\begin{array}{r}\text { Monoclonal } \\
\text { mouse }\end{array}$ & Abcam \\
\hline CNP & $1: 500$ & $5 \mu \mathrm{g}$ & 10 or $12 \%$ & $\begin{array}{r}\text { Monoclonal } \\
\text { mouse }\end{array}$ & Sigma \\
\hline
\end{tabular}




\begin{tabular}{lrrrrr}
\hline PLP/DM20 & $1: 500$ & $0.5 \mu \mathrm{g}$ & $15 \%$ & $\begin{array}{r}\text { Polyclonal } \\
\text { rabbit }\end{array}$ & $\begin{array}{r}\text { K.-A. Nave } \\
\text { (Jung et al., 1996) }\end{array}$ \\
\hline SIRT2 & $1: 500$ & $10 \mu \mathrm{g}$ & 10 or $12 \%$ & $\begin{array}{r}\text { Polyclonal } \\
\text { rabbit }\end{array}$ & Abcam \\
CD9 & $1: 250$ & $0.5 \mu \mathrm{g}$ & $15 \%$ & $\begin{array}{r}\text { Monoclonal } \\
\text { mouse }\end{array}$ & BD Biosciences \\
\hline MAG (513) & $1: 1000$ & $0.5 \mu \mathrm{g}$ & $15 \%$ & $\begin{array}{r}\text { Monoclonal } \\
\text { mouse }\end{array}$ & Chemicon \\
\hline Actin & $1: 2000$ & $5 \mu \mathrm{g}$ & 10 or 12\% & $\begin{array}{r}\text { Monoclonal } \\
\text { mouse }\end{array}$
\end{tabular}

\subsubsection{Secondary antibodies - Immunoblot}

\begin{tabular}{lccc} 
Antibody & Dilution & Species & Source \\
\hline anti-rabbit-HRP & $1: 10000$ & Goat & Dianova \\
\hline anti-mouse-HRP & $1: 10000$ & Goat & Dianova \\
\hline anti-goat-HRP & $1: 1000$ & Donkey & Dianova
\end{tabular}

\subsubsection{Solutions for fixation}

\section{Description}

Avertin

\section{Content}

2,2,2 Tribromethanol 99\%

$2 \%[\mathrm{w} / \mathrm{v}]$

Amylalcohol

$2 \%[\mathrm{v} / \mathrm{v}]$

Mixed at $40^{\circ} \mathrm{C}$ for $30 \mathrm{~min}$ while stirring and subsequently filtered

Stored at $-20^{\circ} \mathrm{C}$

PFA

$16 \%[\mathrm{w} / \mathrm{v}]$

$\mathrm{NaOH}$

$5 \mathrm{~N}$

PFA cooked at $65^{\circ} \mathrm{C}$ for 20 min while stirring, $\mathrm{NaOH}$ droplets until solution was cleared and then filtered 
0.2 M Phosphate buffer

di-Sodiumhydrogenphosphate
$\mathrm{NaCl}$

4\% Paraformaldehyde (PFA)

PFA

Phosphate buffer

PFA

Glutaraldehyde

Phosphate buffer
$0.36 \%[\mathrm{w} / \mathrm{v}]$

$3.1 \%[\mathrm{w} / \mathrm{v}]$

$1 \%[w / v]$

$4 \%[\mathrm{w} / \mathrm{v}]$

$0.1 \mathrm{M}$

$4 \%[\mathrm{w} / \mathrm{v}]$

$2.5 \%[v / v]$

$0.1 \mathrm{M}$

\subsubsection{Immunohistochemistry and staining solutions}

\section{Description}

Citrate buffer (0.01 M, pH 6.0)

Tris buffer $(0.05 \mathrm{M}, \mathrm{pH} 7.6)$

\section{Content}

Citric acid

$1.8 \mathrm{mM}$

Sodium citrate

$8.2 \mathrm{mM}$

Tris/HCl, pH 7.6

$50 \mathrm{mM}$

Sodium chloride

$0.9 \%[\mathrm{w} / \mathrm{v}]$

Phosphate buffer (0.2 M, pH 7.4)

Sodiumdihydrogenphosphate

$0.04 \mathrm{M}$

di-Sodiumhydrogenphosphate

$0.16 \mathrm{M}$

\begin{tabular}{llr} 
PBS/BSA & Sodiumdihydrogenphosphate & $0.04 \mathrm{M}$ \\
& di-Sodiumhydrogenphosphate & $0.16 \mathrm{M}$ \\
& $\mathrm{NaCl}$ & $1.8 \%[\mathrm{w} / \mathrm{v}]$ \\
& Bovine serum albumin (BSA) & $1.0 \%[\mathrm{w} / \mathrm{v}]$ \\
& & \\
Scott's solution & Potassiumhydrogencarbonate & $0.2 \%[\mathrm{w} / \mathrm{v}]$ \\
& Magnesium sulphate & $2 \%[\mathrm{w} / \mathrm{v}]$ \\
\hline $\mathrm{HCl}$ - alcohol & HCl & $0.09 \%[\mathrm{v} / \mathrm{v}]$ \\
& Ethanol & $70 \%[\mathrm{v} / \mathrm{v}]$ \\
\hline \multirow{3}{*}{ Mayer's haematoxylin solution } & Haematoxylin & $0.1 \%[\mathrm{w} / \mathrm{v}]$ \\
& Sodium iodate & $0.02 \%[\mathrm{w} / \mathrm{v}]$ \\
& Potassium aluminum sulfate & $5 \%[\mathrm{w} / \mathrm{v}]$ \\
& Added under constant shaking, solution turned violet \\
& Chloralhydrate & $5 \%[\mathrm{w} / \mathrm{v}]$ \\
& Citric acid & $0.1 \%[\mathrm{w} / \mathrm{v}]$
\end{tabular}




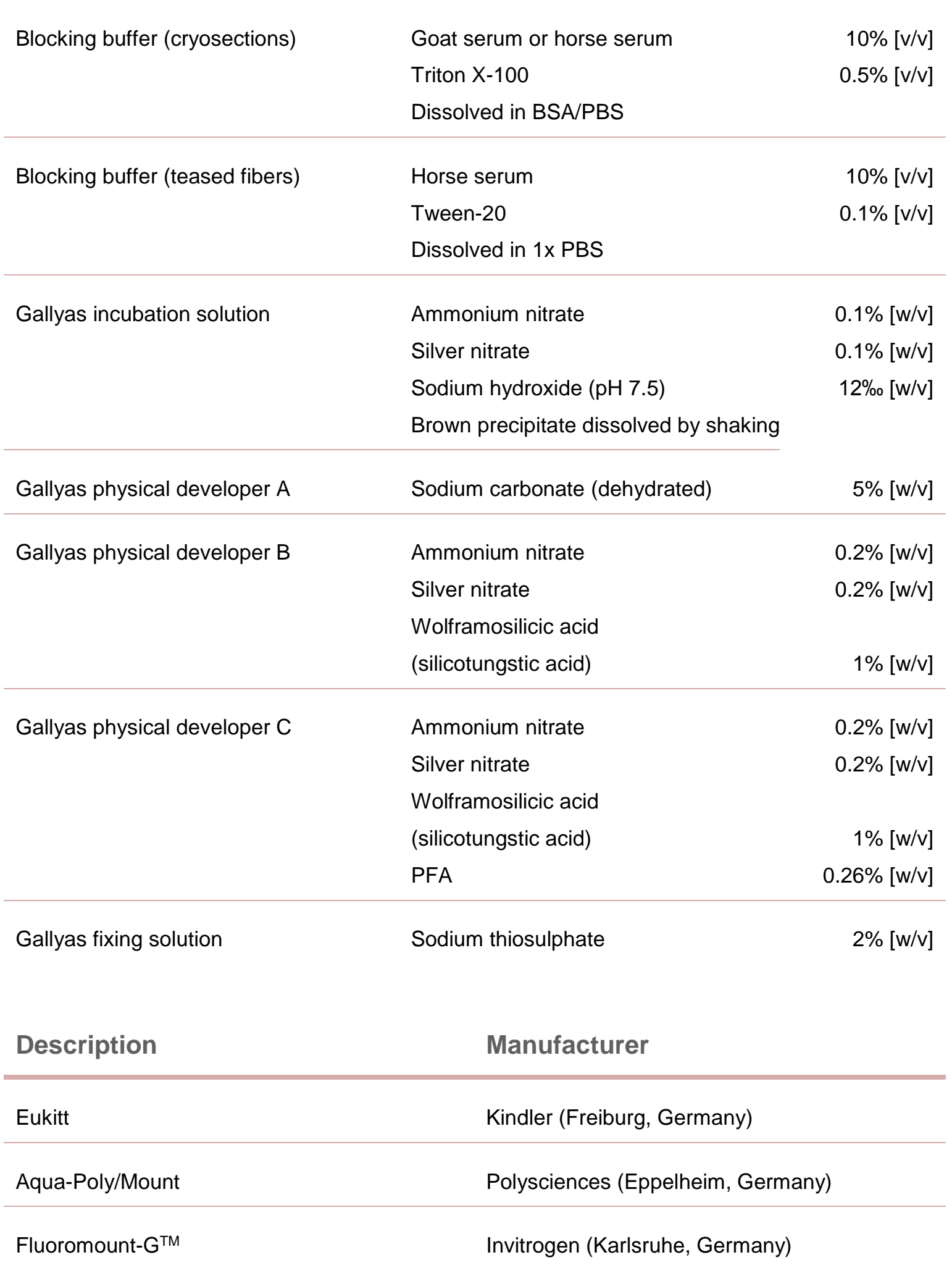




\subsubsection{Primary antibodies - Immunohistochemistry}

\begin{tabular}{lccc} 
Antibody & Dilution & Species & Source \\
\hline ANLN & $1: 200$ & Polyclonal goat & Acris \\
\hline SEPT7 & $1: 1000$ & Polyclonal rabbit & IBL \\
\hline SEPT8 & $1: 500$ & Polyclonal rabbit & PtG \\
\hline MAG (513) & $1: 50$ & Monoclonal mouse & Chemicon \\
\hline BIII-Tubulin (TUJ1) & $1: 1000$ & Monoclonal mouse & Covance \\
\hline NF & $1: 1000$ & Monoclonal mouse & Covance \\
\hline CASPR & $1: 500$ & Monoclonal mouse & Neuromabs \\
\hline Nav1.6 & $1: 500$ & Polyclonal rabbit & Almonelabs
\end{tabular}

\subsubsection{Secondary antibodies - Immunohistochemistry}

\begin{tabular}{lccc} 
Antibody & Dilution & Species & Source \\
\hline anti-mouse-Alexa 488 & $1: 1000$ & Donkey & Dianova \\
\hline anti-rabbit-Alexa 488 & $1: 1000$ & Donkey & Dianova \\
\hline anti-mouse-Alexa 555 & $1: 1000$ & Donkey & Dianova \\
\hline anti-rabbit-Alexa 555 & $1: 1000$ & Donkey & Dianova \\
\hline anti-goat-Cy3 & $1: 500$ & Donkey & Dianova \\
\hline anti-mouse-Dyelight633 & $1: 500$ & Donkey & Dianova
\end{tabular}




\subsubsection{Electron microscopy}

\begin{tabular}{lrr} 
Description & Content & \\
\hline Epon & Glycidether 100 & $171.3 \mathrm{~g}$ \\
& Dodecenyl succinic anhydride (DDSA) & $115 \mathrm{~g}$ \\
& Methyl nadic anhydride (MNA) & $89 \mathrm{~g}$ \\
& Mixed using magnet stirrer for 10 min & \\
& DMP-30 & $6.5 \mathrm{ml}$ \\
& Mixed using magnet stirrer for 20 min & \\
& Na-tetraborat (Borax) & $1 \%[\mathrm{w} / \mathrm{v}]$ \\
Methylene blue & Methylenblau & $1 \%[\mathrm{w} / \mathrm{v}]$ \\
\hline \multirow{3}{*}{ Azure II } & Azure II & $1 \%[\mathrm{w} / \mathrm{v}]$ \\
\hline Methylene blue - Azure II & Methylene blue & $50 \%[\mathrm{v} / \mathrm{v}]$ \\
staining solution & Azure II & $50 \%[\mathrm{v} / \mathrm{v}]$ \\
& Freshly mixed before use &
\end{tabular}

\subsubsection{Solutions for silver impregnation of protein gels}

\begin{tabular}{llr} 
Description & Content & \\
\hline Fixation solution & Ethanol & $40 \%[\mathrm{v} / \mathrm{v}]$ \\
& Acidic acid & $10 \%[\mathrm{v} / \mathrm{v}]$ \\
\hline Sensitization solution & $\mathrm{Na}_{2} \mathrm{~S}_{2} \mathrm{O}_{3}$ & $0.8 \mathrm{mM}$ \\
\hline Staining solution & $\mathrm{AgNO}_{3}$ & $0.2 \%[\mathrm{w} / \mathrm{v}]$ \\
& $\mathrm{HCOH}$ & $0.02 \%[\mathrm{v} / \mathrm{v}]$ \\
\hline Developing solution & $\mathrm{Na}_{2} \mathrm{CO}_{3}$ & $3 \%[\mathrm{w} / \mathrm{v}]$ \\
& $\mathrm{HCOH}$ & $0.02 \%[\mathrm{v} / \mathrm{v}]$ \\
\hline Stop solution & Acidic acid & $5 \%[\mathrm{v} / \mathrm{v}]$
\end{tabular}




\subsubsection{Mouse lines}

Anl $n^{\text {fl/fl }}$ mice (ES cells from EUCOMM, Erwig et al., unpublished)

Sept8 $8^{f / f t l}$ mice (Patzig et al., 2016)

Sept8 ${ }^{\text {null/null }}$ mice (Patzig et al., 2016)

Cnp ${ }^{\text {Cre/Wt }}$ mice (Lappe-Siefke et al., 2003)

Sept9 ${ }^{R 88 W / W t}$ mice (CRISPR, F. Benseler; DNA core facility (MPI EM, Göttingen),

U. Fünfschilling; transgenic service (MPI EM, Göttingen))

Sept2 $2^{f / f l}$ mice

(ES cells from EUCOMM, unpublished)

Sept $9^{f / / f l}$ mice

(Fuchtbauer et al., 2011)

Dhh Cre+ mice

(Jaegle et al., 2003) 


\subsection{Methods}

\subsubsection{Animals}

All animals used in the experiments were bred and kept in the mouse facility of the MaxPlanck-Institute of Experimental Medicine (MPI EM). All mice were sacrificed by cervical dislocation or by perfusion using anesthetics. All experiments were performed in accordance with the German animal welfare law.

\subsubsection{Generation of Anln conditional knockout mice (AnIn cKO)}

To generate ANLN conditional knockout mice (Anln cKO), embryonic stem (ES) cells harboring a modified allele of the Anln gene were obtained from the European Conditional Mouse Mutagenesis Program (EUCOMM). ES cells were injected into FVB blastula derived from C57BL/6N mice and implanted into pseudo-pregnant foster mothers (Animal facility, MPI EM). Positive chimaeras of the ES cell clone EPD0545_1_F09 were bred to wild type C57BL/6N mice gaining F1 offspring. Anln-LacZ animals were identified by genotyping polymerase chain reaction (PCR) and further bred to the Flp-recombinase positive mouse line to remove the LacZ/neo cassette resulting in floxed mice for conditional gene targeting. Homozygous Anln floxed mice $\left(A n l n^{f / f}\right)$ together with heterozygous Cnp mice (Cnp ${ }^{C r e / W t}$ ) (Lappe-Siefke et al., 2003) were used to delete in vivo exon 4 of the Anln gene in oligodendrocytes and Schwann cells specifically. The generation of Anln cKO mice were performed by Ramona Jung (MPI EM) and the animal facility around Ursula Fünfschilling (MPI EM; transgenic service).

\subsubsection{Generation of Sept9R88W/Wt mice}

For the generation of Sept $9^{R 88 W / W t}$ mice, the CRISPR/Cas9 system was used. It was decided to induce a point mutation at the Arginine 88 into a Tryptophan, thereby mimicking a frequent mutation in human hereditary neuralgic amyotrophy (HNA). The implementation of the point mutation using the CRISPR/Cas9 system was achieved by F.Benseler and his group (MPI EM; DAN core facility) and the animal facility around Ursula Fünfschilling (MPI EM; transgenic service).

\subsubsection{Genotyping PCR}

For genotyping, small tail tips or ear punches of P21 young mice were used. They were digested in $180 \mu \mathrm{l} 1 \mathrm{x}$ MGB with $20 \mu \mathrm{l}$ Proteinase $\mathrm{K}$ at $55^{\circ} \mathrm{C}$ with a agitation of about $1250 \mathrm{rpm}$ for $2 \mathrm{~h}$. Afterwards, the Proteinase $\mathrm{K}$ was inactivated by incubation of the tails at $95^{\circ} \mathrm{C}$ for $10 \mathrm{~min}$. Amplification of genotype specific DNA fragments was performed using the polymerase chain reaction (PCR) (Mullis et al., 1986, Saiki et al., 1988). For this purpose, $20 \mu \mathrm{l}$ PCR reaction-mix was added to $1 \mathrm{ml}$ DNA. The PCRs were run in a T3 or 
Gradient Thermocycler (Biometra GmbH, Göttingen, Germany). Primers were selected manually using the DNASTAR Lasergene 9 core suite and then synthesized in house by the service facility of the MPI EM. To separate the PCR products, gels containing $2 \%[\mathrm{w} / \mathrm{v}$ ] agarose in TBE buffer, were used. Before loading $20 \mu \mathrm{l}$ of the PCR samples, $5 \mu \mathrm{l}$ of Gel Red Nucleic Acid Stain (BioTrend (Cologne, Germany); 1:2500 in $\mathrm{dd}_{2} \mathrm{O}$ ) was added for DNA visualization to each sample. The samples were separated at $120 \mathrm{~V}$ for $60 \mathrm{~min}$ in TBE buffer. GeneRuler 100 bp DNA ladder (Thermo Fisher Scientific, St. Leon-Rot, Germany) was used as a marker. For documentation pictures were obtained with an Intas UV system (Intas Science Imaging, Göttingen, Germany). Genotyping of Sept9R88W/Wt mice, was performed by the sequencing facility of F.Benseler (MPI EM; DNA core facility).

PCR reaction Anln gene

\begin{tabular}{lc}
\hline $\mathrm{ddH}_{2} \mathrm{O}$ & $12.2 \mu \mathrm{l}$ \\
GoTaq buffer $(5 \mathrm{x})$ & $4.2 \mu \mathrm{l}$ \\
$\mathrm{dNTP}(2 \mathrm{mM})$ & $2.1 \mu \mathrm{l}$ \\
Primer $29823(10 \mu \mathrm{M})$ & $0.5 \mu \mathrm{l}$ \\
Primer $29824(10 \mu \mathrm{M})$ & $0.5 \mu \mathrm{l}$ \\
Primer $32002(10 \mu \mathrm{M})$ & $0.5 \mu \mathrm{l}$ \\
GoTaq DNA polymerase & $0.07 \mu \mathrm{l}$ \\
\hline
\end{tabular}

$\mathrm{Wt} \sim 570 \mathrm{bp} ;$ Flox $\sim 780 \mathrm{bp} ;$ Rec $\sim 270 \mathrm{bp}$

PCR reaction Sept8 gene

\begin{tabular}{lr}
\hline $\mathrm{ddH}_{2} \mathrm{O}$ & $12.4 \mu \mathrm{l}$ \\
GoTaq buffer $(5 \mathrm{x})$ & $4.2 \mu \mathrm{l}$ \\
$\mathrm{dNTP}(2 \mathrm{mM})$ & $2.1 \mu \mathrm{l}$ \\
Primer $16458(10 \mu \mathrm{M})$ & $0.1 \mu \mathrm{l}$ \\
Primer $16459(10 \mu \mathrm{M})$ & $0.6 \mu \mathrm{l}$ \\
Primer $16460(10 \mu \mathrm{M})$ & $0.6 \mu \mathrm{l}$ \\
GoTaq DNA polymerase & $0.07 \mu \mathrm{l}$ \\
\hline
\end{tabular}

Wt $\sim 450$ bp; Flox 550 bp; Rec 260 bp) 
PCR reaction Cnp gene

\begin{tabular}{|c|c|}
\hline $\mathrm{ddH}_{2} \mathrm{O}$ & $11.3 \mu l$ \\
\hline GoTaq buffer (5x) & $4.2 \mu l$ \\
\hline dNTP (2 mM) & $2.1 \mu l$ \\
\hline Primer $4192(10 \mu \mathrm{M})$ & $0.2 \mu \mathrm{l}$ \\
\hline Primer $4193(10 \mu \mathrm{M})$ & $0.2 \mu l$ \\
\hline Primer $2016(10 \mu \mathrm{M})$ & $1 \mu l$ \\
\hline Primer $7315(10 \mu \mathrm{M})$ & $1 \mu l$ \\
\hline GoTaq DNA polymerase & $0.07 \mu \mathrm{l}$ \\
\hline \multicolumn{2}{|c|}{$\mathrm{Wt} \sim 700 \mathrm{bp} ; \mathrm{Cre}+\sim 450 \mathrm{bp}$} \\
\hline \multicolumn{2}{|c|}{ PCR reaction Sept9 gene } \\
\hline $\mathrm{ddH}_{2} \mathrm{O}$ & $11.9 \mu \mathrm{l}$ \\
\hline GoTaq buffer $(5 x)$ & $4.2 \mu l$ \\
\hline dNTP (2 mM) & $2.1 \mu \mathrm{l}$ \\
\hline Primer $23844(10 \mu \mathrm{M})$ & $1 \mu l$ \\
\hline Primer $23845(10 \mu \mathrm{M})$ & $0.2 \mu \mathrm{l}$ \\
\hline Primer $23846(10 \mu \mathrm{M})$ & $0.6 \mu l$ \\
\hline GoTaq DNA polymerase & $0.07 \mu \mathrm{l}$ \\
\hline \multicolumn{2}{|c|}{ Wt $~ 520$ bp; Flox 600 bp; Rec 650 bp } \\
\hline \multicolumn{2}{|c|}{ PCR reaction Dhh Cre } \\
\hline $\mathrm{ddH}_{2} \mathrm{O}$ & $12.7 \mu \mathrm{l}$ \\
\hline GoTaq buffer (5x) & $4.2 \mu \mathrm{l}$ \\
\hline dNTP (2 mM) & $2.1 \mu \mathrm{l}$ \\
\hline Primer $10967(10 \mu \mathrm{M})$ & $0.5 \mu \mathrm{l}$ \\
\hline Primer $15793(10 \mu \mathrm{M})$ & $0.5 \mu l$ \\
\hline GoTaq DNA polymerase & $0.07 \mu \mathrm{l}$ \\
\hline
\end{tabular}


PCR reaction Mbp gene

\begin{tabular}{lr}
\hline $\mathrm{ddH}_{2} \mathrm{O}$ & $12.5 \mu \mathrm{l}$ \\
GoTaq buffer $(5 \mathrm{x})$ & $4.2 \mu \mathrm{l}$ \\
$\mathrm{dNTP}(2 \mathrm{mM})$ & $2.1 \mu \mathrm{l}$ \\
Primer $1879(10 \mu \mathrm{M})$ & $0.4 \mu \mathrm{l}$ \\
Primer $30833(10 \mu \mathrm{M})$ & $0.4 \mu \mathrm{l}$ \\
Primer $30834(10 \mu \mathrm{M})$ & $0.2 \mu \mathrm{l}$ \\
Primer $30835(10 \mu \mathrm{M})$ & $0.2 \mu \mathrm{l}$ \\
GoTaq DNA polymerase & $0.07 \mu \mathrm{l}$ \\
\hline Wt $\sim 170$ bp; Mbp- 380 bp &
\end{tabular}

\subsubsection{RNA isolation and analysis}

For RNA isolation and subsequent analysis the corpus callosum of $n=6$ mice (male and female, 4 mo of age) was collected. The corpus callosum was dissected using a brain slicer. From each brain section the corpus callosum was collected manually. The collected tissue was directly incubated in RNAlater ${ }^{\mathrm{TM}}$ solution (Ambion ${ }^{\circledR}$, Thermo Fisher Scientific, St. LeonRot, Germany), at $4^{\circ} \mathrm{C}$ o/n and then stored at $-20^{\circ} \mathrm{C}$ until usage. RNA isolation, cDNA synthesis, and qRT-PCR were done by Ursula Kutzke.

\subsubsection{RNA isolation}

Small scale RNA isolation from separated corpus callosum of male mice was performed using Qiagen's "RNeasy Mini Prep" kit. The kit is based on a selective binding of RNAs bigger than 200 bases to a silica-gel based membrane under high-salt conditions, which excludes binding of $5 \mathrm{~S}, 5.8 \mathrm{~S}$ and tRNAs. RNA isolation and purification was carried out following the manufacturer's instructions. The tissue was homogenized in Trizol (Life Technologies $^{\mathrm{TM}}$, Thermo Fisher Scientific, St. Leon-Rot, Germany), followed by chloroform extraction $(200 \mu \mathrm{l}$ chloroform was added to $0.6 \mathrm{ml}$ homogenate). After $15 \mathrm{~min}$ of centrifugation at $16000 \times g$ (Heraeus Biofuge Pico table centrifuge, $13000 \mathrm{rpm}$ ) the upper aqueous phase was transferred to a new $2 \mathrm{ml}$ tube. One volume of ethanol was added to the samples. Then the samples were mixed and applied to RNeasy columns. After $1 \mathrm{~min}$ centrifugation at $16000 \times g$ the columns were washed one time with the RW1 buffer and two times with the RPE buffer. RNA was eluted with $50 \mu$ of RNase-free $d_{d d} \mathrm{H}_{2} \mathrm{O}$. The quality of RNA was tested using the Agilent RNA 6000 Nano KIT and the Agilent 2100 Bioanalyzer 
(Agilent Technologies, Santa Clara, California, United States), following the company's instructions. The amount of RNA was measured using the NanoDrop 2000 Spectrophotometer (Thermo Fisher Scientific, St. Leon-Rot, Germany) and the RNA concentration for all samples was adjusted to $100 \mathrm{ng} / \mu \mathrm{l}$.

\subsubsection{2 cDNA synthesis}

As a next step, the isolated RNA was transcribed into a complementary single stranded DNA (cDNA) in a reverse transcription reaction catalyzed by RNA dependent DNA polymerase SuperScript III (Invitrogen, Karlsruhe, Germany).

\section{cDNA synthesis protocol}

$8 \mu \mathrm{l} \quad \mathrm{RNA}$ (800 ng total)

$2 \mu \mathrm{l} \quad \mathrm{dT}$ mix Primer $(0.6 \mathrm{pmol} / \mu \mathrm{l})$

$2 \mu \mathrm{l} \quad \mathrm{N} 9$ (random nonamers $120 \mathrm{pmol} / \mu \mathrm{l}$ )

To denaturate RNA and primers, the mixture was incubated for $10 \mathrm{~min}$ at $70^{\circ} \mathrm{C}$ and $1 \mathrm{~min}$ on ice.

Add following premix to the reaction:

$4 \mu \mathrm{l} \quad 5 \mathrm{x}$ first strand buffer

$1 \mu \mathrm{l} \quad \mathrm{dNTP}(10 \mathrm{mM})$

$2 \mu \mathrm{l}$ DDT $(100 \mathrm{mM})$

$1 \mu \mathrm{l}$ SuperScript III reverse transcriptase $(200 \mathrm{U} / \mu \mathrm{l})$

Incubation in thermocycler with the following settings:

$25^{\circ} \mathrm{C} \quad 10 \mathrm{~min}$

$50^{\circ} \mathrm{C} \quad 45 \mathrm{~min}$

$55^{\circ} \mathrm{C} \quad 45 \mathrm{~min}$

Synthesized cDNA was diluted $1: 30$ with $\mathrm{dd}_{2} \mathrm{O}$ and stored at $-20^{\circ} \mathrm{C}$. 


\subsubsection{Quantitative real-time PCR (qRT-PCR)}

The pipetting robot epMotion 5075 (Eppendorf, Hamburg, Germany) was used for pipetting. qRT-PCR was performed using the Power SYBR Green PCR Master Mix (Promega, Fitchburg, Wisconsin, United States) and the Light Cycler 480 II (Roche Diagnostics GmbH, Mannheim, Germany).

Mixture for gene expression analysis

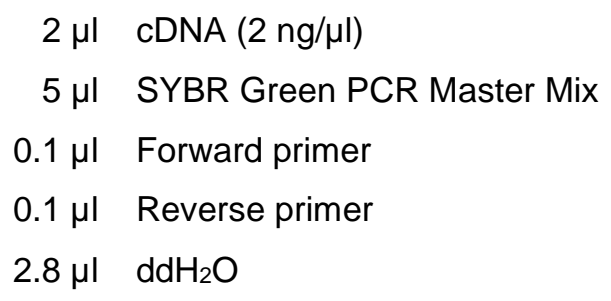

All reactions were performed in quadruplicates.

\section{Program for 50 cycles}

$\begin{array}{ll}15 \mathrm{sec} & 95^{\circ} \mathrm{C} \\ 1 \mathrm{~min} & 60^{\circ} \mathrm{C}\end{array}$

mRNA abundance was analyzed in relation to the mean of the standard peptidylprolyl isomerase A (Ppia), which did not differ between genotypes. Statistical analysis was performed using GraphPad Prism 6.

\subsubsection{Biochemical protein analysis}

\subsubsection{Sample collection}

Brains of male mice were dissected at P75. The optic nerves were removed at the chiasma, as well as the brainstem underneath the cerebellum. The brains were cut in half and directly frozen on dry ice and stored at $-80^{\circ} \mathrm{C}$ until usage.

Sciatic nerves of male mice were dissected at P75. The nerves were collected from the distal end without muscle tissue, up to the proximal end where the nerve enters the backbone. The sciatic nerves were directly frozen on dry ice and stored at $-80^{\circ} \mathrm{C}$ until usage.

\subsubsection{Myelin purification}

The myelin purification was adapted from a protocol of Norton and Poduslo (Norton and Poduslo, 1973, Larocca and Norton, 2007). Briefly, all supplies were precooled and 
procedure was performed on ice or at $4^{\circ} \mathrm{C} . \mathrm{n}=3$ mouse brains per genotype were used. For myelin purification, half brains were homogenized in $5 \mathrm{ml} 0.32 \mathrm{M}$ sucrose containing protease inhibitor (Roche Diagnostics $\mathrm{GmbH}$, Mannheim, Germany) using the IKA T10 basic Ultra-Turrax (Speed 3). For PIP2 measurements, additionally to the protease inhibitor also phosphatase inhibitor (Roche Diagnostics $\mathrm{GmbH}$, Mannheim, Germany) was added. From the homogenate, $200 \mu \mathrm{l}$ were stored at $-80^{\circ} \mathrm{C}$ for further analysis. For density gradient centrifugation, the homogenate was carefully overlaid on $6 \mathrm{ml} 0.85 \mathrm{M}$ sucrose and centrifuged for $30 \mathrm{~min}$ at $75000 \times \mathrm{g}$ in a swing out rotor TH641 (Thermo Fisher Scientific, St. Leon-Rot, Germany) using the Sorvall WX+ Ultra series centrifuge (Thermo Fisher Scientific, St. Leon-Rot, Germany). The roughly purified myelin accumulated at the interphase and was transferred into a new tube. Water was added to the purified myelin and centrifuged again for $15 \mathrm{~min}$ at $75000 \times \mathrm{g}$. The pellet was re-suspended in $\mathrm{dd}_{2} \mathrm{O}$, incubated for $10 \mathrm{~min}$ on ice and then centrifuged at $12000 \mathrm{xg}$ for $15 \mathrm{~min}$. This so-called osmotic shock was repeated a second time. Afterwards the pellet was re-suspended in $5 \mathrm{ml}$ $0.32 \mathrm{M}$ sucrose (containing protease inhibitor (additionally phosphatase inhibitor for $\mathrm{PIP}_{2}$ measurements)) and carefully overlaid on $6 \mathrm{ml} 0.85 \mathrm{M}$ sucrose for a second density gradient centrifugation (30 min at $75000 \times$ g). Further purified myelin again accumulated at the interphase and was transferred into a new tube. Water was added to the myelin and centrifuged again for $15 \mathrm{~min}$ at $75000 \mathrm{x}$. The pellet containing a myelin enriched fraction (further referred as purified myelin) was re-suspended in $200 \mu \mathrm{l}$ xTBS including protease inhibitor (additionally phosphatase inhibitor for $\mathrm{PIP}_{2}$ measurements) and was stored at $-80^{\circ} \mathrm{C}$ (detailed protocol in Erwig et al., accepted).

\subsubsection{Lysate preparation (PNS)}

Of $n=3$ animals per genotype both sciatic nerves were taken from $-80^{\circ} \mathrm{C}$ and then cut on dry ice. Homogenization was performed in $400 \mu$ RIPA buffer using Precellys (Peqlab, Erlangen, Germany). The nerves got lysed two times with the following adjustments: $6500 \mathrm{rpm} 3 \times 10 \mathrm{sec}+10 \mathrm{sec}$ break. After each time the developed foam was removed by short centrifugation (Heraeus Biofuge Pico table centrifuge, $13000 \mathrm{rpm}$ ) and the lysate was kept on ice for further $10 \mathrm{~min}$. In the next step the lysate was transferred into a $1.5 \mathrm{ml}$ tube and centrifuged at $13000 \mathrm{rpm}$ for $10 \mathrm{~min}$ at $4^{\circ} \mathrm{C}$. The supernatant was then transferred to a new tube and stored at $-80^{\circ} \mathrm{C}$.

\subsubsection{Protein concentration determination and sample preparation}

To determine the protein concentration of purified myelin, brain lysate, or sciatic nerve lysate, the Lowry assay was performed (Lowry et al., 1951; Peterson, 1979) using the BioRad DC Protein Assay kit. The kit was used according to the manufacturer's 'microplate 
assay'-protocol. The optical density was measured at $650 \mathrm{~nm}$ using the Eon ${ }^{\mathrm{TM}} \mathrm{High}$ Performance Microplate Spectrophotometer (BioTek, Vermont, USA).

The obtained protein concentrations were used to set up samples for SDS PAGE separation. The samples were adjusted to $0.1 \mu \mathrm{g} / \mu \mathrm{l}$ and to $1 \mu \mathrm{g} / \mu \mathrm{l}$ including $1 \times$ SDS sample buffer and $5 \% \beta$-mercaptoethanol $[\mathrm{v} / \mathrm{v}]$ to denature the proteins. To detect myelin associated glycoprotein (MAG) and CD9, non-reduced conditions without $\beta$-mercaptoethanol were used (protein concentration set to $0.1 \mu \mathrm{g} / \mu \mathrm{l}$ ). The samples were heated for $10 \mathrm{~min}$ at $40^{\circ} \mathrm{C}$ and afterwards kept at $-20^{\circ} \mathrm{C}$. Before usage the samples were always put to $40^{\circ} \mathrm{C}$ for $10 \mathrm{~min}$.

\subsubsection{Protein separation using SDS PAGE}

Acrylamid gels $(8 \%, 10 \%, 12 \%$, or $15 \%[\mathrm{w} / \mathrm{v}])$ were prepared using the Bio-Rad system. The gels were casted between $1.5 \mathrm{~mm}$ thick spacer plates and thin coverplates. First the SDS separating gel was casted and overlaid with isopropanol to get a smooth border. The

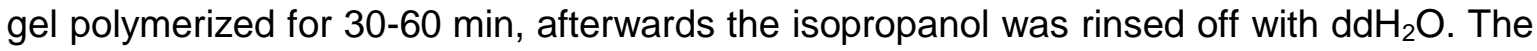
remaining water was removed with filter papers and the SDS stacking gel was casted on top of the SDS separating gel. A plastic comb (10 well) was used to obtain pockets for protein samples. After another $30 \mathrm{~min}$ of polymerization, the gels were used immediately or stored in wet papers at $4^{\circ} \mathrm{C}$, not longer than 5 days.

Bio-Rad chambers were used to assemble the gels and the plastic combs were removed. The pockets were washed with $1 \times$ Laemmli running buffer while filling up the chamber. The samples were pipetted carefully into the pockets, whereby different amounts of protein were loaded to obtain optimal signals for each antibody detection (see 3.1.8 Primary antibodies Immunoblot). Proteins were separated by constant current (200 V) for $1 \mathrm{~h}$ (until the blue $1 \mathrm{x}$ SDS front runs out of the SDS separating gel) using a Bio-Rad power supply. Afterwards the gels were removed from the coverplates and incubated in transfer buffer for $15 \mathrm{~min}$ before transferring the proteins to a polyvinylidene difluoride (PVDF) membrane.

\subsubsection{Immunoblot - semi-dry}

For immunodetection, proteins were transferred to a PVDF membrane as described by Towbin and colleagues (Towbin et al., 1979). The PVDF membrane was activated in $100 \%$ ethanol $[\mathrm{v} / \mathrm{v}]$ for $1 \mathrm{~min}$, washed two times shortly with $\mathrm{ddH}_{2} \mathrm{O}$ and kept in transfer buffer for 10 min. For the semi-dry blot the Novex® Semi-Dry Blotter (Invitrogen, Karlsruhe, Germany) was used. On the anode plate four Whatman ${ }^{\mathrm{TM}}$ papers (GE Healthcare Life Science, Buckinghamshire, UK) soaked in ice cold transfer buffer were placed. To avoid air bubbles in the stack, the Whatman ${ }^{\mathrm{TM}}$ papers were rolled with a blotting roller. Afterwards the activated PVDF membrane was placed on top, followed by the gel and four more Whatman ${ }^{\mathrm{TM}}$ paper (soaked with ice cold transfer buffer). Proteins were transferred at $20 \mathrm{~V}$ 
for 45 min using a Bio-Rad power supply. Up to four gels could be transferred simultaneously.

\subsubsection{Immunoblot - wet tank}

To transfer big proteins (>100 kDa) a wet tank blot was performed. The PVDF membrane was activated as described for the semi-dry immunoblot. The membrane was incubated in wet-tank transfer buffer (methanol 20\% [v/v]) for $10 \mathrm{~min}$. For blotting the Bio-Rad system was used. Between the brace, a stack of 3 thick Whatman ${ }^{\mathrm{TM}}$ paper, the activated membrane, the SDS separating gel, and further 3 thick Whatman ${ }^{\mathrm{TM}}$ paper was build. The Whatman ${ }^{\mathrm{TM}}$ paper were sucked with ice cold transfer buffer and the whole stack was built together in the buffer. After removing air bubbles with a blotting roller, the brace was clapped together and put in the chamber. The chamber was placed in an ice bucked and filled with pre-cooled transfer buffer. Proteins were transferred by constant current at $100 \mathrm{~V}$ for $1.5 \mathrm{~h}$ using a Bio-Rad power supply.

\subsubsection{Immunodetection of blotted proteins}

After blotting, the PVDF membrane was incubated in blocking buffer $(5 \%$ [w/v] non-fat dry milk in TBST) at $\mathrm{RT}^{\circ} \mathrm{C}$ for $30-60$ min. The primary antibodies were diluted in $5 \mathrm{ml}$ blocking buffer in a $50 \mathrm{ml}$ Falcon® tube (see 3.1.8 Primary antibodies - Immunoblot). The membrane was incubated in these tubes on a rotor at $4^{\circ} \mathrm{C}$ o/n or over two nights. To increase the binding of some antibodies (e.g. Anillin), the membrane was incubated for further $2 \mathrm{~h}$ at $\mathrm{RT}^{\circ} \mathrm{C}$. Afterwards, the membranes were washed in TBST $3 \times 5 \mathrm{~min}$ and then incubated in diluted HRP coupled secondary antibodies (in blocking buffer) for $1-2 \mathrm{~h}$ on a rotor at $\mathrm{RT}^{\circ} \mathrm{C}$. The membranes were than washed $3 \times 5 \mathrm{~min}$ in TBST. Membranes were carefully picked up with forceps and placed onto a glass plate. Enhanced chemiluminescence detection $(E C L)$ solution was added according to the manufacturer's recommendations (Western Lightning® Plus-ECL or WesternBright Chemiluminescence Substrat Sirius). After 1 min of incubation, membranes were placed between two plastic foils and chemiluminescence was scanned for one time $1 \mathrm{~min}$ and afterwards for 15 min using the Intas ChemoCam system (Intas Science Imaging, Göttingen, Germany).

\subsubsection{Quantitative Mass Spectrometry}

For quantitative mass spectrometry (MS), samples with a protein content of at least $100 \mu \mathrm{g}$

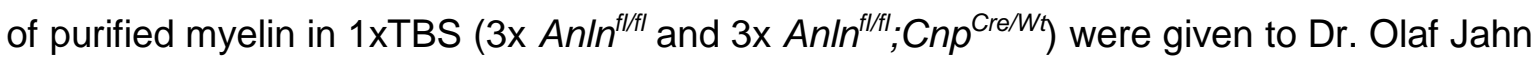
(MPI EM; proteomics unit). Probes were analyzed using DRE-HD-MSE, as described in (Erwig et al., accepted). Experiments were performed using three biological replicates each 
in two technical replicates. Final quantifications, statistical analysis, and diagrams were prepared with Microsoft Excel 2013 and GraphPad Prism 6.

\subsubsection{Histological analysis}

\subsubsection{Perfusion fixation of mouse tissue}

Mice were anesthetized by injection of avertin $(0.2 \mathrm{ml}$ per $10 \mathrm{~g}$ of body weight) interperitoneally (i.p.). The successful anesthesia was confirmed by testing reflexes using a pinch in the hind paws. Afterwards the mice were stabilized with the ventral side up and the abdomen was opened. To expose the heart the diaphragm was removed, so a butterfly cannula (27G, Venofix), connected to a peristaltic pump, could be injected in the left ventricle, while the right atrium was cut. The blood was flushed out with $37^{\circ} \mathrm{C}$ warm Hank's Balanced Salt Solution (HBSS) (Invitrogen, Karlsruhe, Germany) for 5 min. Afterwards the HBSS was replaced by $4 \%$ PFA fixative. To fix the tissue, the fixation run for 10 min (40$50 \mathrm{ml}$ 4\% PFA).

\subsubsection{Embedding for cryosections}

Either freshly dissected optic nerves or perfused brains and spinal cords were embedded. Therefore, the tissue was incubated in $4 \%$ PFA for $1 \mathrm{~h}$ at $4^{\circ} \mathrm{C}$ and then transferred to $10 \%$ [w/v] sucrose in $0.1 \mathrm{M}$ phosphate buffer for $1 \mathrm{~h}$ at $4^{\circ} \mathrm{C}$. Afterwards, the tissue was kept in $20 \%\left[\mathrm{w} / \mathrm{v}\right.$ ] sucrose in $0.1 \mathrm{M}$ phosphate buffer at $4^{\circ} \mathrm{C} \mathrm{o} / \mathrm{n}$. After further o/n incubation in $30 \%$ $[\mathrm{w} / \mathrm{v}]$ sucrose in $0.1 \mathrm{M}$ phosphate buffer at $4^{\circ} \mathrm{C}$ the tissue was embedded in Tissue-Tek ${ }^{\circledR}$ O.C.T. ${ }^{\text {TM }}$ Compound (Sakura, Staufen, Germany) and afterwards stored at $-20^{\circ} \mathrm{C}$. For sectioning, the Reichert Jung ${ }^{\circledR}$ Cryocut 1800 Cryostat (Leica, Wetzlar, Germany) was used. $10 \mu \mathrm{m}$ thick sections were collected on Superfrost ${ }^{\circledR}$ Plus microscope slides (Thermo Fisher Scientific, St. Leon-Rot, Germany) and dried at $\mathrm{RT}^{\circ} \mathrm{C}$. For storage, the slides were kept at $-20^{\circ} \mathrm{C}$.

\subsubsection{Immunohistochemistry - cryosections}

The following protocol was used for immunohistochemistry of cryosections:

\begin{tabular}{lll} 
Purpose & Reagent & Conditions \\
\hline Permeabilization & Methanol & 3 min at $\mathrm{RT}^{\circ} \mathrm{C}$ \\
\hline Washing & $1 \times$ PBS & $3 \times 5$ min at $\mathrm{RT}^{\circ} \mathrm{C}$ \\
\hline Blocking & Blocking buffer (3.1.11) & $1 \mathrm{~h}$ at $\mathrm{RT}^{\circ} \mathrm{C}$ \\
\hline
\end{tabular}




\begin{tabular}{|c|c|c|c|}
\hline 1. Antibody & Diluted in blocking buffer & $\mathrm{o} / \mathrm{n}$ at $4^{\circ} \mathrm{C}$ & \\
\hline Washing & 1x PBS & $3 \times 5 \mathrm{~min}$ & \\
\hline 2. Antibody & Diluted in blocking buffer & $1-2 \mathrm{~h}$ at $\mathrm{RT}^{\circ} \mathrm{C}$ & \multirow{7}{*}{$\begin{array}{l}\frac{\pi}{0} \\
\frac{0}{0} \\
0\end{array}$} \\
\hline Washing & 1x PBS & 2x 10 min at $\mathrm{RT}^{\circ} \mathrm{C}$ & \\
\hline DAPI & DAPI 1:50000 in 1x PBS & 10 min at $\mathrm{RT}^{\circ} \mathrm{C}$ & \\
\hline Washing & 1x PBS & 2x 10 min at $\mathrm{RT}^{\circ} \mathrm{C}$ & \\
\hline Washing & $\mathrm{ddH}_{2} \mathrm{O}$ & $2 \times 2$ min at $\mathrm{RT}^{\circ} \mathrm{C}$ & \\
\hline Mounting & $\begin{array}{l}\text { Aqua-Poly/Mount or } \\
\text { Fluoromount-GTM }\end{array}$ & at $\mathrm{RT}^{\circ} \mathrm{C}$ & \\
\hline Storage & & $4^{\circ} \mathrm{C}$ & \\
\hline
\end{tabular}

\subsubsection{Imaging and analysis of fluorescent staining}

Slides were imaged with the confocal microscope Leica SP5. Signal was collected sequentially with the objective HCX PL APO CS $63.0 \times 1.30 \mathrm{GLYC} 21^{\circ} \mathrm{C}$ UV. An argon laser with the excitation of $488 \mathrm{~nm}$ was used to excite the Alexa 488 fluorophore and the emission was set to 500-560 nm. Cy3 was excited with the DPSS 561 laser at an excitation of $561 \mathrm{~nm}$ and the emission was set to $573-630 \mathrm{~nm}$. Also the Alexa 555 fluorophore was excited with the DPSS 561 laser, and the emission was set to $575-630 \mathrm{~nm}$. The HeNe laser was used to excite Dyelight633 with $633 \mathrm{~nm}$ and emission was detected between 645-738 nm. DAPI was excited with $405 \mathrm{~nm}$ and collected between 417-480 $\mathrm{nm}$. The LAS AF lite software was used to export the images as TIF files. The original pictures of images in Figure 8A and B, were partially provided by Julia Patzig. The Imaris software was used for 3Dreconstructions.

For the quantifications of nodal density, the frequency of occurrence of two CASPRimmunopositive paranodes was analyzed using the ImageJ software. CASPRimmunopositivity was converted using a threshold and counted using ITNC plugin ( $n=4$ animals per genotype, 1 section each, 1 micrograph analyzed (size $16000 \mu \mathrm{m}^{2}$ ). Statistical analysis was performed using GraphPad Prism 6.0.

The node and paranode length (indicated in Figure 16) was assessed using the ImageJ software. For paranode length the mean of both paranodes beside an axon were calculated. 
$\mathrm{n}=4$ animals per genotype, 1 section each, 5 random micrographs of spinal cord white matter. Statistical analysis was performed using GraphPad Prism 6.0.

\subsubsection{Embedding for paraffin sections}

Mice were perfused with $4 \%$ PFA and post-fixed o/n at $4^{\circ} \mathrm{C}$. Before embedding, brains were divided coronal into 3 parts using a brain slicer. For thin sectioning, the tissue was embedded in paraffin (Paraplast, Leica, Wetzlar, Germany) using the automated system HMP 110 ((Microm, Walldorf, Germany) with the following program:

\begin{tabular}{ll} 
Procedure & Time \\
\hline $50 \%[\mathrm{v} / \mathrm{v}]$ Ethanol & 1 hour \\
$70 \%[\mathrm{v} / \mathrm{v}]$ Ethanol & $2 \times 2$ hours \\
$96 \%[\mathrm{v} / \mathrm{v}]$ Ethanol & $2 \times 2$ hours \\
$100 \%[\mathrm{v} / \mathrm{v}]$ Ethanol & $2 \times 2$ hours \\
Isopropanol & 1 hour \\
Xylol & $2 \times 2$ hours \\
Paraffin & $2 \times 2$ hours
\end{tabular}

Afterwards, the tissue was placed into metal forms with the sectioning side facing the ground. The tissue was then casted with $60^{\circ} \mathrm{C}$ warm paraffin and put on a pre-cooled plate for hardening (Paraffin embedding station: MICROM AP280 (Microm, Walldorf, Germany). The blocks were removed and stored at $\mathrm{RT}^{\circ} \mathrm{C}$. The tissue was cut in $5 \mu \mathrm{m}$ thick sections using a microtome (HM 400, MICROM (Microm; Walldorf, Germany) and dried o/n at $37^{\circ} \mathrm{C}$. The sections were stored at $\mathrm{RT}^{\circ} \mathrm{C}$ until further usage. The sectioning was partially done by Annette Fahrenholz.

\subsubsection{Immunohistochemistry - paraffin sections}

Before usage, the sections were deparaffinized by the following steps:

Procedure

$60^{\circ} \mathrm{C}$

Xylol

Xylol/Isopropanol (1:1)

$100 \%[v / v]$ Ethanol

$90 \%[\mathrm{v} / \mathrm{v}]$ Ethanol

$70 \%[v / v]$ Ethanol

$50 \%[\mathrm{v} / \mathrm{v}]$ Ethanol

$\mathrm{ddH}_{2} \mathrm{O}$

\section{Time}

$10 \min$

2x $10 \mathrm{~min}$

$10 \mathrm{~min}$

$5 \mathrm{~min}$

$5 \min$

$5 \min$

$5 \mathrm{~min}$

$5 \mathrm{~min}$ 
For permeabilization of the $5 \mu \mathrm{m}$ thick sections, the slides were incubated in citrate buffer for $5 \mathrm{~min}$ and then cooked for $10 \mathrm{~min}$ in boiling citrate buffer (650 Watts in microwave oven). After cooling for $20 \mathrm{~min}$, the sections were placed in Tris buffer containing $2 \%$ [w/v] non-fat dry milk. Afterwards the slides were placed into Shandon Coverplates ${ }^{\mathrm{TM}}$ (Thermo Fisher Scientific, St. Leon-Rot, Germany). These coverplates ensure an even distribution of small volume amounts of antibody-dilutions. The slides were again rinsed with Tris buffer containing $2 \%[\mathrm{w} / \mathrm{v}]$ non-fat dry milk. Afterwards, as horse radish peroxidase (HRP) coupled secondary antibodies were used, the endogenous peroxidase activity was inhibited by applying 3\% [v/v] hydrogen peroxide for $5 \mathrm{~min}$. After washing with Tris buffer containing $2 \%[\mathrm{w} / \mathrm{v}]$ non-fat dry milk, the slides were incubated in blocking solution (20\% [v/v] goat serum in BSA/PBS) for 30 min at $R T^{\circ} \mathrm{C}$. Primary antibodies were diluted in BSA/PBS and incubated $\mathrm{o} / \mathrm{n}$ at $4^{\circ} \mathrm{C}$. On the next day, the slides were washed with Tris buffer containing $2 \%[\mathrm{w} / \mathrm{v}]$ non-fat dry milk. For staining of amyloid beta precursor protein (APP), ionized calcium-binding adapter molecule 1 (IBA1), and glial fibrillary acidic protein (GFAP) the LSAB2 kit (Dako, Hamburg, Germany) was used. The sections were incubated with $100 \mu \mathrm{l}$ of bridging antibody i.e. a biotinylated secondary antibody for 10 min at $\mathrm{RT}^{\circ} \mathrm{C}$ and rinsed with Tris buffer containing $2 \%[\mathrm{w} / \mathrm{v}]$ non-fat dry milk. The HRP complex bound antibody was incubated for $10 \mathrm{~min}$ at $\mathrm{RT}^{\circ} \mathrm{C}$. For staining of macrophages/activated microglia (MAC3), sections were incubated with an a-rat-biotinylated antibody (Vector Labs, Burlingame, California, United States) for $30 \mathrm{~min}$. The two components of the Vector Elite ABC kit (Vector Labs) were mixed and the slides incubated for $30 \mathrm{~min}$. The coverplates were removed and all sections were rinsed with Tris buffer without non-fat dry milk. The HRP substrate 3,3'-Diaminobenzidine (DAB) was applied by using the DAB Zytomed Kit (Zytomed Systems GmbH, Berlin, Germany) and incubated for 10 min. Brown labeling appeared where antibodies recognized their targets. After additional washing with $\mathrm{dd}_{2} \mathrm{O}$, sections were incubated in $0.1 \%$ [w/v] Haematoxylin for 5 min to label nuclei. Blue coloration appears due to the interaction with the basic nuclear compartment. Sections were rinsed with $\mathrm{ddH}_{2} \mathrm{O}$, incubated with $\mathrm{HCl}$-Alcohol for 5-10 sec and with Scott's solution for $5 \mathrm{~min}$. This was followed by additional rinse with $\mathrm{ddH}_{2} \mathrm{O}$. The tissue was dehydrated by the following steps:

\begin{tabular}{ll} 
Procedure & Time \\
\hline $50 \%[\mathrm{v} / \mathrm{v}]$ Ethanol & $5 \mathrm{~min}$ \\
$70 \%[\mathrm{v} / \mathrm{v}]$ Ethanol & $5 \mathrm{~min}$ \\
$90 \%[\mathrm{v} / \mathrm{v}]$ Ethanol & $5 \mathrm{~min}$ \\
$100 \%[\mathrm{v} / \mathrm{v}]$ Ethanol & $5 \mathrm{~min}$ \\
Xylol/lsopropanol $(1: 1)$ & $5 \mathrm{~min}$ \\
Xylol & $2 \times 5 \mathrm{~min}$
\end{tabular}


In the end, slides were mounted using Eukitt (Kindler, Freiburg, Germany). The stainings were partially done by Annette Fahrenholz.

\subsubsection{Imaging and quantification of DAB developed stainings}

The DAB stained sections were imaged using the Zeiss Axio Z1 with the Zen 2011 software (Zeiss, Oberkochern, Germany). The quantification of GFAP, IBA1, and MAC3 signal was done using an ImageJ plugin for semi-automated analysis. First, the color threshold was used to transform the colored picture to a black and white picture; brown signal represented by positive black signal. Second, the area of the positive black signal was measured and related to the size of the analyzed area. Diagrams, quantification and Student's t-test ( $p$ value) were prepared with GraphPad Prism 6.

The APP positive spheroids were counted manually, using the ImageJ software. Diagrams, quantification and statistical analysis were prepared with GraphPad Prism 6.

For all quantifications, the hippocampal fimbria was selected. For each animal, the average of both fimbriae was calculated. Data were normalized to wild type levels. All quantifications were performed blinded to the genotype. The quantifications were performed at P75 with an $n=5$ per genotype.

\subsubsection{Gallyas silver impregnation}

Deparaffinized $10 \mu \mathrm{m}$ brain sections (coronal) and cerebellum sections (sagital) were used. The technique developed by Gallyas is based on binding of colloidal silver particles to myelinated fibers (Gallyas, 1979). The silver impregnation has been done by Annette Fahrenholz. To visualize myelin, the following protocol has been used:

\begin{tabular}{lll} 
Purpose & Reagent & Incubation time \\
\hline $\begin{array}{l}\text { Blocking of non- } \\
\text { myelin tissue }\end{array}$ & Pyridine/acetic acid $(2: 1)$ & $30 \mathrm{~min}$ \\
\hline Washing & $\mathrm{ddH}_{2} \mathrm{O}$ & $3 \times 10 \mathrm{~min}$ \\
\hline Incubation & Incubation solution & $\begin{array}{l}1 \mathrm{~min} 100 \mathrm{Watts} \\
\text { microwave } \\
10 \text { min } \mathrm{RT}^{\circ} \mathrm{C}\end{array}$ \\
\hline Washing & $0.5 \%[\mathrm{v} / \mathrm{v}]$ Acetic acid & $3 \times 5$ min \\
\hline
\end{tabular}




\begin{tabular}{lll}
\hline Developing & $\begin{array}{l}\text { Physical developer: } \\
100 \mathrm{ml} \text { Solution A }\end{array}$ & $3-15 \mathrm{~min}$ \\
& $\begin{array}{l}70 \mathrm{ml} \text { Solution B } \\
30 \mathrm{ml} \text { Solution } \mathrm{C}\end{array}$ & \\
& $1.0 \%[\mathrm{v} / \mathrm{v}]$ Acetic acid & $3 \times 5 \mathrm{~min}$ \\
\hline Stop reaction & $\mathrm{ddH}_{2} \mathrm{O}$ & $2 \times 3 \mathrm{~min}$ \\
\hline Washing & $2 \%[\mathrm{v} / \mathrm{v}]$ Sodium thiosulfate & $5 \mathrm{~min}$ \\
\hline Stabilization & $50 \%[\mathrm{v} / \mathrm{v}]$ Ethanol & $5 \mathrm{~min}$ \\
Dehydartion & $70 \%[\mathrm{v} / \mathrm{v}]$ Ethanol & $5 \mathrm{~min}$ \\
& $90 \%[\mathrm{v} / \mathrm{v}]$ Ethanol & $5 \mathrm{~min}$ \\
& $100 \%[\mathrm{v} / \mathrm{v}]$ Ethanol & $5 \mathrm{~min}$ \\
& Xylol/lsopropanol $(1: 1)$ & $5 \mathrm{~min}$ \\
& Xylol
\end{tabular}

The slides were in the end mounted using Eukitt (Kindler, Freiburg, Germany). Images were captured at 10x magnification (Zeiss Axiolmager Z1) and stitched using Zeiss Zen2011. Images in Figure 18A and $A^{\prime}$ are representative for $n=3$ mice per genotypes at P75.

\subsubsection{Electron microscopy (EM)}

\subsubsection{Tissue preparation - Electron microscopy}

Animals were sacrificed by cervical dislocation and optic nerves were carefully removed at the chiasm. Sciatic nerves were dissected, whereby at the distal side a piece of muscle was kept attached for future identification of the distal end. The freshly dissected nerves were directly incubated in Karlsson-Schultz fixative (K\&S) and stored at $4^{\circ} \mathrm{C}$ until further processing.

\subsubsection{Epon embedding}

For EM imaging, the fixed nerves were embedded using an automated system (EMPT, Leica, Wetzlar, Germany). Before embedding the nerves were washed with $0.1 \mathrm{M}$ phosphate buffer and placed into plastic chambers. The tissue was processed using the following protocol: 


\begin{tabular}{lcr} 
Solution & Incubation time & Temperature \\
\hline Phosphate buffer & $3 \times 10 \mathrm{~min}$ & $4^{\circ} \mathrm{C}$ \\
\hline $2 \%[\mathrm{w} / \mathrm{v}] \mathrm{OsO}_{4}$ & $4 \mathrm{~h}$ & $4^{\circ} \mathrm{C}$ \\
\hline $\mathrm{ddH}_{2} \mathrm{O}$ & $3 \times 10 \mathrm{~min}$ & $4^{\circ} \mathrm{C}$ \\
\hline $30 \%[\mathrm{v} / \mathrm{v}]$ Ethanol & $20 \mathrm{~min}$ & $4^{\circ} \mathrm{C}$ \\
\hline $50 \%[\mathrm{v} / \mathrm{v}]$ Ethanol & $20 \mathrm{~min}$ & $4^{\circ} \mathrm{C}$ \\
\hline $70 \%[\mathrm{v} / \mathrm{v}]$ Ethanol & $20 \mathrm{~min}$ & $4^{\circ} \mathrm{C}$ \\
\hline $90 \%[\mathrm{v} / \mathrm{v}]$ Ethanol & $20 \mathrm{~min}$ & $4^{\circ} \mathrm{C}$ \\
\hline $100 \%[\mathrm{v} / \mathrm{v}]$ Ethanol & $4 \times 10 \mathrm{~min}$ & $4^{\circ} \mathrm{C}$ \\
\hline Propylenoxid & $3 \times 10 \mathrm{~min}$ & $\mathrm{RT}^{\circ} \mathrm{C}$ \\
\hline Propylenoxid/Epon $2: 1$ & $2 \mathrm{~h}$ & $\mathrm{RT}^{\circ} \mathrm{C}$ \\
\hline Propylenoxid/Epon $1: 1$ & $2 \mathrm{~h}$ & $\mathrm{RT}^{\circ} \mathrm{C}$ \\
\hline Propylenoxid/Epon $1: 2$ & $4 \mathrm{~h}$ & $\mathrm{RT}^{\circ} \mathrm{C}$ \\
\hline Epon & $4 \mathrm{~h}$ & \\
\hline
\end{tabular}

The tissue was then placed into molds filled with Epon. A label was put within the Epon for identification of the probe and left $\mathrm{o} / \mathrm{n}$ at $60^{\circ} \mathrm{C}$ for Epon polymerization.

\subsubsection{Sectioning of Epon embedded tissue}

For semi-thin sectioning the embedded sciatic nerves were cut into $500 \mathrm{~nm}$ thin sections. This was done with a microtome (Ultracut S, Leica, Wetzlar, Germany) and a diamond knife (Diatome Ultra 45). The sections were collected on water and then transferred onto an object slide (Marienfeld with help of a Pasteur pipette. The object slide was then put on a $60{ }^{\circ} \mathrm{C}$ hot plate to dry the sections.

To analyze the embedded optic and sciatic nerves on electron microscope level, $50 \mathrm{~nm}$ thin sections were cut using the same equipment as for the semi-thin sections. The sections were collected on formvar polyvinyl coated double sized slot grids $(2 \mathrm{~mm}-1 \mathrm{~mm}$, AGAR scientific, Essex, UK). 


\subsubsection{Staining of semi-thin sections}

$500 \mathrm{~nm}$ thin sections of sciatic nerves were stained with Methylene blue-Azure II solution (Richardson et al., 1960). On a $60^{\circ} \mathrm{C}$ hot plate, sections were incubated for $1 \mathrm{~min}$ in the staining solution and then extensively rinsed with $\mathrm{ddH}_{2} \mathrm{O}$. After drying, the tissue was mounted using Eukitt and imaged with a 100x oil objective of the Zeiss Axio Imager Z1 (Zeiss, Oberkochern, Germany).

\subsubsection{Contrasting of ultra-thin sections}

For proper contrasting of ultra-thin sections, the formvar polyvinyl coated double sized slot grids were placed upside-down on drops of the following solutions:

\begin{tabular}{lr} 
Solution & Incubation time \\
\hline Uranyl acetate & 30 min (kept dark) \\
$\mathrm{ddH}_{2} \mathrm{O}$ & $3 \times 1 \mathrm{~min}$ \\
Reynolds lead citrate & $6 \mathrm{~min}$ \\
$\mathrm{dd}_{2} \mathrm{O}$ & $6 \times 1 \mathrm{~min}$
\end{tabular}

Alternatively, the sections carrying grids were placed upside-down on a drop of UranyLess (Electron Microscopy Science, Hatfield, Panama) for 30 min and then washed 6 times with $\mathrm{dd}_{2} \mathrm{O}$.

\subsubsection{Analysis of EM pictures}

Ultra-thin sections were analyzed with the Zeiss EM900 (Zeiss, Oberkochen, Germany) and digital pictures were obtained using the wide-angle dual speed 2K-CCD-Camera (TRS, Moorenweis, Germany). g-ratio measurements and quantifications of myelin abnormalities were performed using the ImageJ software. Microsoft Excel 2013 was used for calculations of the g-ratio. Diagrams, quantification and statistical analysis were prepared with GraphPad Prism 6. All quantifications were performed blinded to the genotype. Presentation of pictures was prepared using Adobe Photoshop CS6 and Adobe Illustrator CS6.

The g-ratio was calculated as the ratio between the axonal Feret diameter and the Feret diameter of the corresponding myelin sheath (Figure 19A). To determine g-ratios, one quarter $\left(55 \mu \mathrm{m}^{2}\right)$ of at least 15 images was quantified, yielding a minimum of 200 myelinated axons per animal. $n=5$ per genotype at 6 mo of age. The g-ratio was assessed using GraphPad Prism 6.0. For calculation, only normal appearing myelinated axons were 
analyzed (Figure 8), to ensure that possible changes in myelin thickness could be related to the loss of ANLN and are not cause of other pathological effects.

For assessment of $\%$ of myelinated axons and $\%$ of degenerating/degenerated profiles, mice were analyzed at an age of 6 mo. 4-5 male mice were used per genotype. 15 randomly selected, non-overlapping images were taken per optic nerve at $7000 \times$ magnification ( 1 field $=220 \mu \mathrm{m}^{2}$ ). Electron micrographs were analyzed using ImageJ. A minimum of 1600 axons per animal were assigned to one of three categories: healthy-appearing myelinated axons, healthy-appearing non-myelinated axons, and degenerating/degenerated axons (partially shown in Figure 8). Axons were counted as myelinated if ensheathed by at least one complete layer of compacted myelin. Degenerating/degenerated axons were identified by tubovesicular structures, amorphous cytoplasm, axonal spheroids or absence of an identifiable axon within a myelin sheath, respectively.

The area occupied by myelin outfoldings was quantified by a point counting method (Edgar et al., 2009) at different time points (P14, P75, 6 mo; $n=4-6$ per genotype). Briefly, a regular grid of $0.25 \mu \mathrm{m}^{2}$ was placed on the images. The number of intercepts coinciding with myelin outfoldings was related to the evaluated area. Significance was determined using GraphPad Prism 6.0.

To identify the axon diameter frequency distribution, one quarter $\left(55 \mu \mathrm{m}^{2}\right)$ of at least 15 images was quantified, yielding the axon diameter of at least 200 up to 650 myelinated axons per animal. The images for Cnp heterozygous and SEPT8 deficient mice and their corresponding controls were kindly provided by Ulrike Gerwig (CNP) and Julia Patzig (SEPT8). $n=5$ for all genotypes and all time points. The diameter was calculated using ImageJ, therefore the axon was encircled and the Feret diameter was calculated by the software. The frequency distribution was visualized using GraphPad Prism 6.0, the bin size was set to 0.2 and each replicate was binned. Significance was determined using GraphPad Prism 6.0 (Kolmogorov-Smirnov Test). All quantifications were performed blinded to the genotype. 


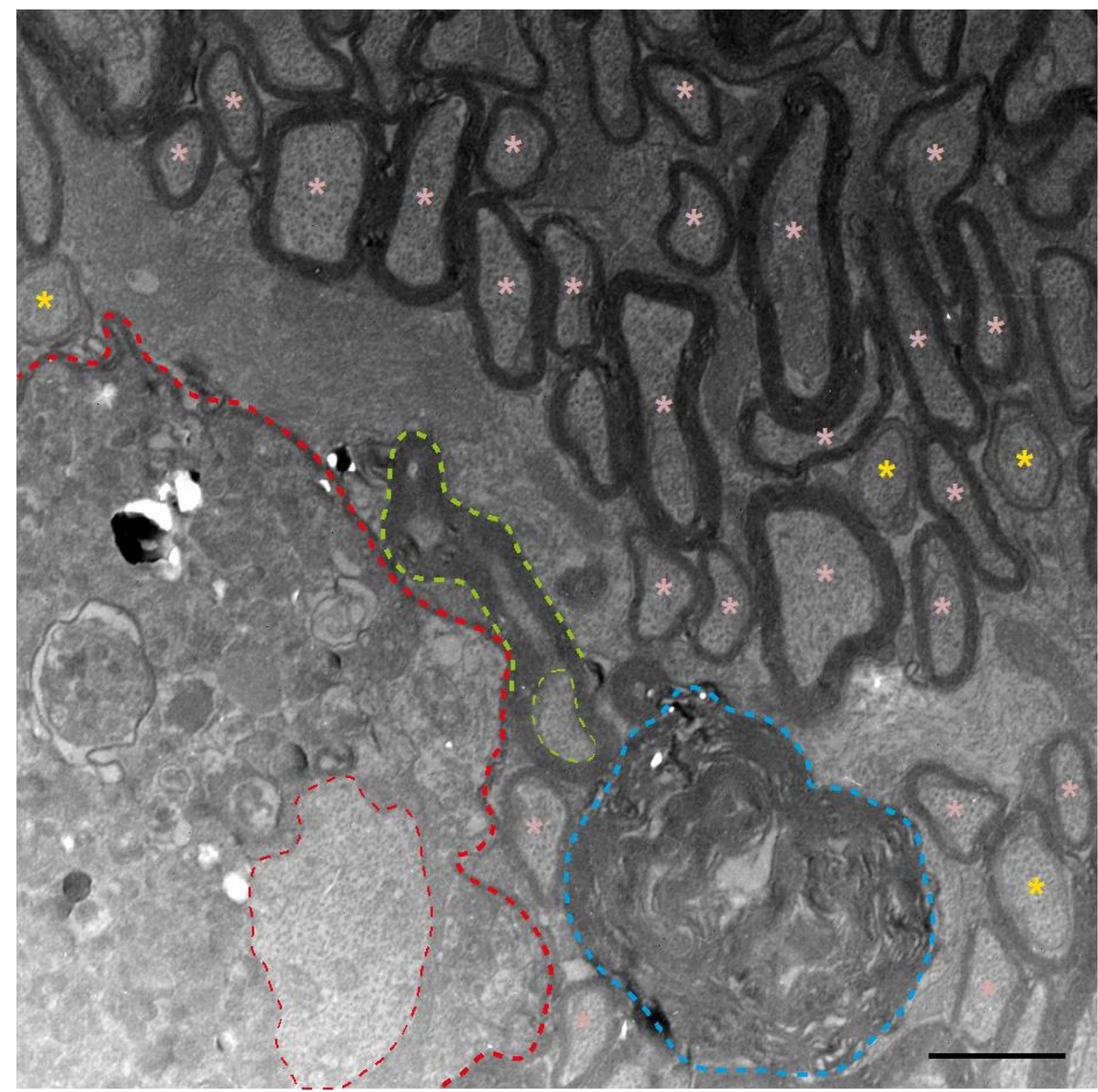

- = - Outfolding

- - Axonal spheroid

- - - Degenerated Profile

* Axon at node of Ranvier or paranodal region

* Normal appearing myelinated axon

Figure 8: Overview of profiles found in electron micrographs. Electron micrograph of an Anln cKO optic nerve cross section at 6 mo of age. The image exemplifies different kinds of detected profiles within an optic nerve. The profile marked in red is showing an axonal spheroid, whereby the light line surrounds the axon and the bold line is marking the corresponding compact myelin. The blue marked profile surrounds redundant myelin, where no axon was identifiable within myelin sheaths. Green marks an axon with an outfolding, whereby the light line surrounds the axon and the bold line surrounds the outfolding. Axons marked with a yellow asterisk show axons that are likely at a node of Ranvier or paranodal region, as they are surrounded by a thin layer of compact myelin and in addition the axons are surrounded by a thicker layer of cytoplasm (King, 2013). Axons marked with a light red asterisk are classified as normal appearing myelinated axons, as they show no signs of pathology. Scale bare: $1 \mu \mathrm{m}$ 


\subsubsection{Focused ion beam scanning electron microscopy (FIB SEM)}

The following steps were done by the EM facility of the MPI EM. Anna Steyer prepared the samples and processed all steps to obtain images. Christos Nardis segmented the images for the 3D reconstruction.

\subsubsection{Sample preparation}

Mouse optic nerves were fixed in $4 \%$ formaldehyde and $2.5 \%$ glutaraldehyde (Science Services) in $0.1 \mathrm{M} \mathrm{PB}$ for at least $24 \mathrm{~h}$. The samples were processed following a modified OTO (Deerinck et al., 2010) protocol. The samples were washed in 0.1 M PB (3 x $15 \mathrm{~min})$ and then incubated for $3 \mathrm{~h}$ at $4{ }^{\circ} \mathrm{C}$ in $2 \% \mathrm{OsO}_{4}$ (Electron Microscopy Sciences) and $0.25 \%$ $\mathrm{K}_{4}\left[\mathrm{Fe}(\mathrm{CN})_{6}\right]$ (Electron Microscopy Sciences). The nerves were washed with $\mathrm{H}_{2} \mathrm{O}(3 \times 15$ $\min$ ) and were incubated with $0.1 \%$ thiocarbohydrazide (Sigma-Aldrich) for $1 \mathrm{~h}$ at room temperature. To enhance the contrast further the tissue was treated with $2 \% \mathrm{OsO}_{4}$ for $90 \mathrm{~min}$ at room temperature. The samples were washed with $\mathrm{H}_{2} \mathrm{O}(3 \times 15 \mathrm{~min})$ and contrasted overnight with $2 \%$ uranyl acetate (SPI-Chem) at $4{ }^{\circ} \mathrm{C}$. The samples were washed again with $\mathrm{H}_{2} \mathrm{O}(3 \times 15 \mathrm{~min})$, followed by dehydration in an increasing acetone series $(30 \%, 50 \%, 75 \%, 90 \%, 3 \times 100 \%)$. The tissue was infiltrated with increasing concentrations of Durcupan (Sigma-Aldrich, components A, B, C) 2 h each (25\%, $50 \%$, $75 \%$ Durcupan in acetone) and then incubated in $100 \%$ Durcupan o/n. Fresh Durcupan with accelerator (component D) was added to the samples for $5 \mathrm{~h}$, before embedding the samples in resin blocks. The blocks were polymerized for $48 \mathrm{~h}$ at $60^{\circ} \mathrm{C}$.

\subsubsection{FIB SEM procedure}

Samples were trimmed with a $90^{\circ}$ diamond trimming knife (Diatome AG, Biel, Switzerland). The blocks were attached to the SEM stub (Science Services GmbH, Pin $12.7 \mathrm{~mm}$ x $3.1 \mathrm{~mm}$ ) by a silver filled epoxy (Epoxy Conductive Adhesive, EPO-TEK EE 1294; EMS) and polymerized at $60^{\circ}$ overnight. The samples were coated with a $10 \mathrm{~nm}$ platinum layer using the sputter coating machine EM ACE600 (Leica) at $35 \mathrm{~mA}$ current. Samples were placed into the Crossbeam 540 focused ion beam scanning electron microscope (Carl Zeiss Microscopy $\mathrm{GmbH}$ ). To ensure even milling and to protect the surface, a $400 \mathrm{~nm}$ platinum layer was deposited on top of the region of interest. Atlas 3D (Atlas 5.1, Fibics, Canada) software was used to collect the $3 \mathrm{D}$ data. Samples were exposed with a $15 \mathrm{nA}$ current and a $7 \mathrm{nA}$ current was used to polish the surface. The images were acquired at $1.5 \mathrm{kV}$ with the ESB detector (450 V ESB grid, pixel size $x / y 2 \mathrm{~nm}$ ) in a continuous mill and acquire mode using 700 pA for the milling aperture (z-step $50 \mathrm{~nm}$ ). 


\subsubsection{Image analysis}

Image alignments were done with TrakEM2 (Cardona et al., 2012), a plugin of Fiji (Schindelin et al., 2012). The following post-processing steps were performed in Fiji. The dataset was cropped, inverted and a local contrast enhancement (CLAHE) was applied. The images were manually segmented using IMOD (Kremer et al., 1996).

\subsubsection{Electrophysiology}

Nerve conduction velocity in the CNS was measured in vivo on 6 month old male mice (2 Anl $n^{f / f t}, 9$ controls (WT from (Patzig et al., 2016)), 7 Anln cKO). Anl $/ n^{f / f l}$ mice and controls were pooled after testing significance (not significant; two-tailed unpaired t-test; $p=0.8399$ ). Measurements were performed by Dr. Payam Dibaj (MPI EM) essentially as described (Dibaj et al., 2012, Patzig et al., 2016).

\subsubsection{1 $\mathrm{PIP}_{2}$ measurement}

Purified myelin of $n=6$ male mice at P75 were sent to Prof. Dr. Ingo Heilmann (Halle, Germany). Lipid extraction followed by combined thin layer chromatography and gas chromatography was used to measure PIP 2 levels (Goebbels et al., 2010, Konig et al., 2008). 


\section{Results}

\subsection{The ANLN/septin filament in CNS myelin}

\subsubsection{Anillin and septins co-localize in CNS white matter}

To identify the localization of ANLN in relation to myelin septins, immunohistochemical triple-stainings were performed on cryosections of mouse optic nerves (longitudinal) and mouse brains (coronal). As reference, different known markers of myelin or axons were used. MAG (Cyan) is a known marker of the adaxonal myelin membrane (Trapp and Quarles, 1982, Trapp et al., 1989), where the septin filament is localized as well (Patzig et al., 2016). The markers NF (neurofilament) and TUJ1 ( $\beta$ III-Tubulin) were used to visualize axons (Cyan). ANLN (red) was found to co-localize to SEPT8 (green) in longitudinal cryosections of optic nerves (Figure 9A), showing a similar filament-like labeling as SEPT8 (arrowheads). The same co-localization was detected in white matter tracts of coronal cryosections of mouse brains (Figure 9B). Filaments (arrowheads) along axons were found in the fimbria (b-b') as well as in the corpus callosum ( $b$ "). Images in A and B were obtained together with Dr. Julia Patzig.

For 3-dimensional (3D) reconstruction, z-stacks of fluorescent immunohistochemical stainings of longitudinal spinal cord cryosections were generated via confocal laser scanning microscopy (Figure 9C and D). To reconstruct the confocal z-stacks into 3D, the Imaris software was used. Figure 9C shows maximal projections of confocal z-stacks and a 3D reconstruction of SEPT7 (green) and an axonal marker (NF; red). The images display the known filament-like assembly of SEPT7 along the axon, as seen in Patzig et al. (2016). Figure 9D shows maximal projections of confocal z-stacks and a 3D reconstruction of ANLN and the axonal marker NF. The images illustrate a filament-like structure of ANLN along the axon, as well as a patchy pattern all over the axon.

Figure 9E shows the protein abundance of ANLN and SEPT8 in brain lysate and purified myelin. A myelin marker (MOG) and an axonal marker (TUJ1) were loaded as controls for the responsive fraction. The abundance of MOG is strongly enriched in purified myelin, whereas the abundance of TUJ1 is decreased. ANLN was only detectable in purified myelin, supporting the localization in mouse brain white matter.

Taken together, the results suggest a similar distribution of ANLN along the axon, as previously found for septins. Additionally, ANLN localizes to parts of the axon without SEPT8 co-localization. 
A
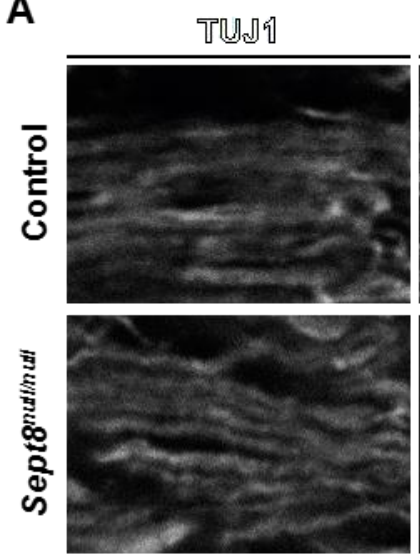

B

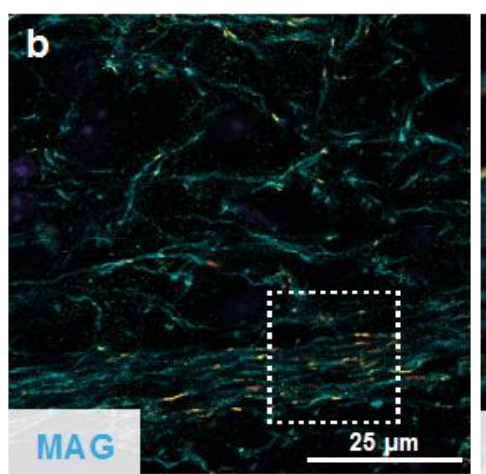

\section{Spinal cord reconstruction}

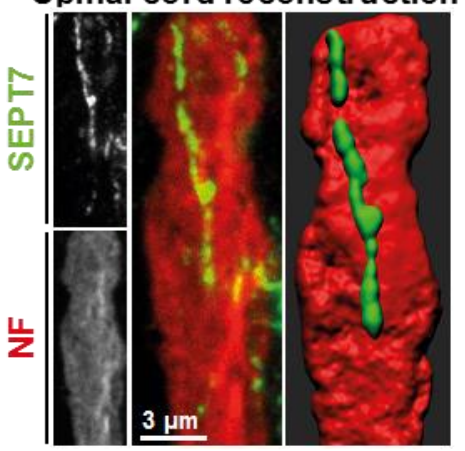

SEPT8
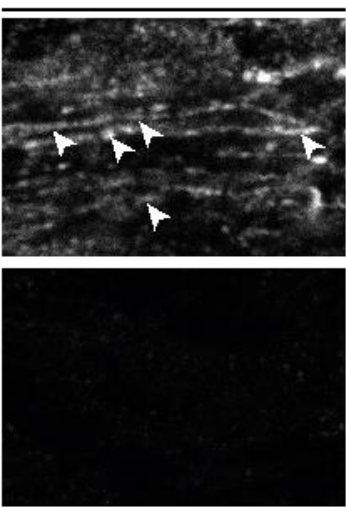

Brain, coronal ANLN SEPT8


\section{E}

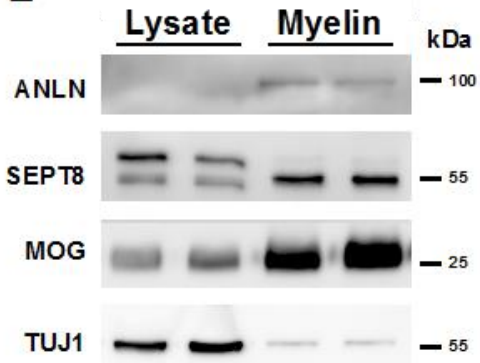

Figure 9: Localization of the ANLN/septin filaments in mouse CNS. (A) Immunolabeling validates close proximity (arrowheads) of ANLN (red) with SEPT8 (green) in myelinated fiber tracts of wild type (Wt) mice. Immunolabeling of Sept8null/null mice indicates diminishment of ANLN similar to SEPT8. Longitudinally sectioned optic nerves of P75 mice are shown. TUJ1 (white) was labeled as an axonal control. Images are representative of three independent experiments. (B) Immunolabeling of coronal brain sections of P75 Wt mice detects ANLN (red) and SEPT8 (green) filaments (arrowheads) in white matter tracts. b-b' shows labeling in the fimbria. In b' a blow up of the dashed square in $\mathbf{b}$ is shown. b" shows labeling in the corpus callosum. MAG/NF were labeled as control. (C) Immunofluorescent signal of SEPT7 (green) extends longitudinally along axons (neurofilamentlabeling (red)). Images display maximal projections of confocal z-stacks and a 3-dimensional reconstruction of longitudinally sectioned spinal cord of P75 Wt mice. Images are representative of three animals. (D) Immunofluorescent signal of ANLN (green) partially extends longitudinally and partially patchy along axons (neurofilament-labeling (red)). Images display maximal projections of confocal z-stacks and a 3-dimensional reconstruction of longitudinally sectioned spinal cord of P75 control mice $\left(A n / n^{f / f f}\right)$. Images are representative of three animals. (E) Immunoblot analysis of brain lysates and myelin purified from P75 control mice $\left(A n / n^{f / f}\right)$. It is of note that ANLN is only detectable in purified myelin. MOG and TUJ1 were detected as controls. Images in A and $B$ were obtained together with Dr. Julia Patzig. 


\subsubsection{ANLN and septins appear similar in development}

To possibly discover the chronology of appearance of ANLN and septins, the abundances of these proteins were analyzed in mice at different developmental states. Immunohistochemical triple-stainings were performed on longitudinal cryosections of mouse optic nerves at P15, P21, and P28 (Figure 10A, Images were obtained together with Dr. Julia Patzig). ANLN and SEPT8 were found to appear around P21. Each filament-like labeling was positive for both, ANLN and SEPT8. At P28 the filament-like labeling was seen more often compared to P21. In Figure 10B, immunoblots on mouse brain myelin illustrate the abundance of ANLN and CNS myelin septins at P15, P18, P21, and P24. ANLN appears in the same pattern as septins, showing an increasing abundance up to P21.

Taken together, every filament-like labeling shows both, ANLN and septin labeling. Further, the abundance of ANLN and septins increases in the same pattern, suggesting that both, ANLN and septins are needed to form a cytoskeletal structure.

A

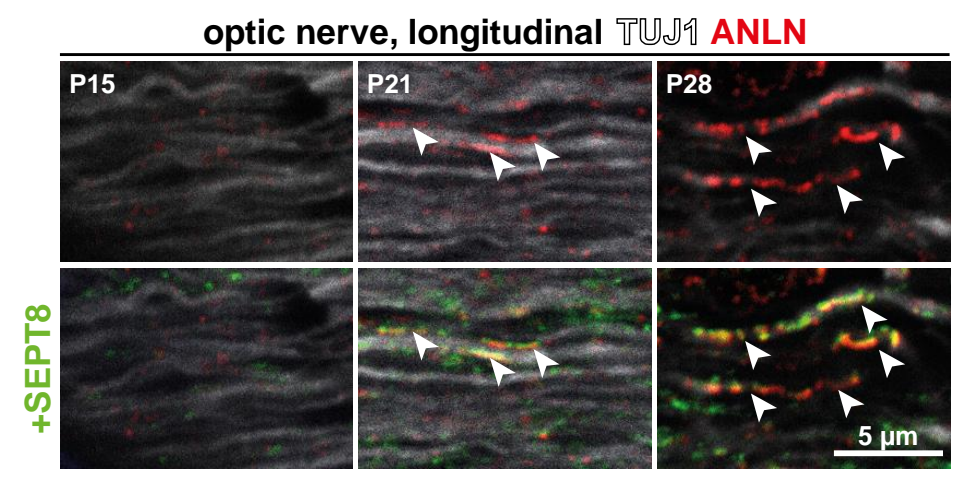

B

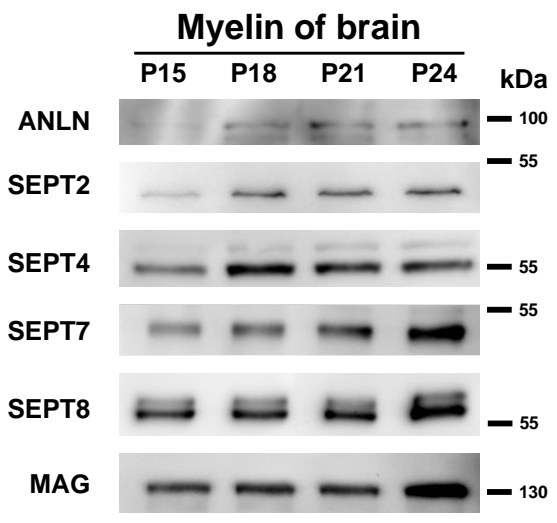

Figure 10: ANLN and septins in development. (A) Immunolabeling of wild type optic nerves detects ANLN (red) partially at P21 and at P28 but not at P15. Note that every filamentous ANLN labeling is in close proximity to SEPT8 (green) labeling, indicated by arrowheads. TUJ1 was labeled as an axonal marker. Images are representative of three experiments. Images were obtained together with Dr. Julia Patzig (B) Immunoblotting indicates that the abundance of ANLN and septins (SEPT2, SEPT4, SEPT7, SEPT8) increases with age in myelin purified from wild type brains at P15, P18, P21, and P24, reflecting the maturation of myelin. L-MAG was detected as control.

\subsection{Characterization of $A n / n^{f / f t} ; C p^{C r e / W t}$ mice}

\subsubsection{Generation of mice lacking ANLN from myelinating glia cells}

As described in 3.2.2, Anln cKO mice were generated (Ramona Jung (MPI EM) and Ursula Fünfschilling (MPI EM, transgenic service)). The scheme in Figure 11A depicts the ordered construct (ES cells ordered from EUCOMM) and the conditional deletion of the Anln gene. Figure 11B illustrates the results of the genotyping PCR (described in 3.2.4). The upper row depicts that only in presence of the Cre recombinase a product of about $350 \mathrm{bp}$ is present. In the middle row, it is depicted that the loxP sites increase the PCR product arising from 
primer 1 (P1) and primer 2 (P2). In the lower row, a product from P1 and primer 4 (P4) arises solely in the AnIn cKO mice. The binding site of each primer is depicted in Figure $11 \mathrm{~A}$.

A
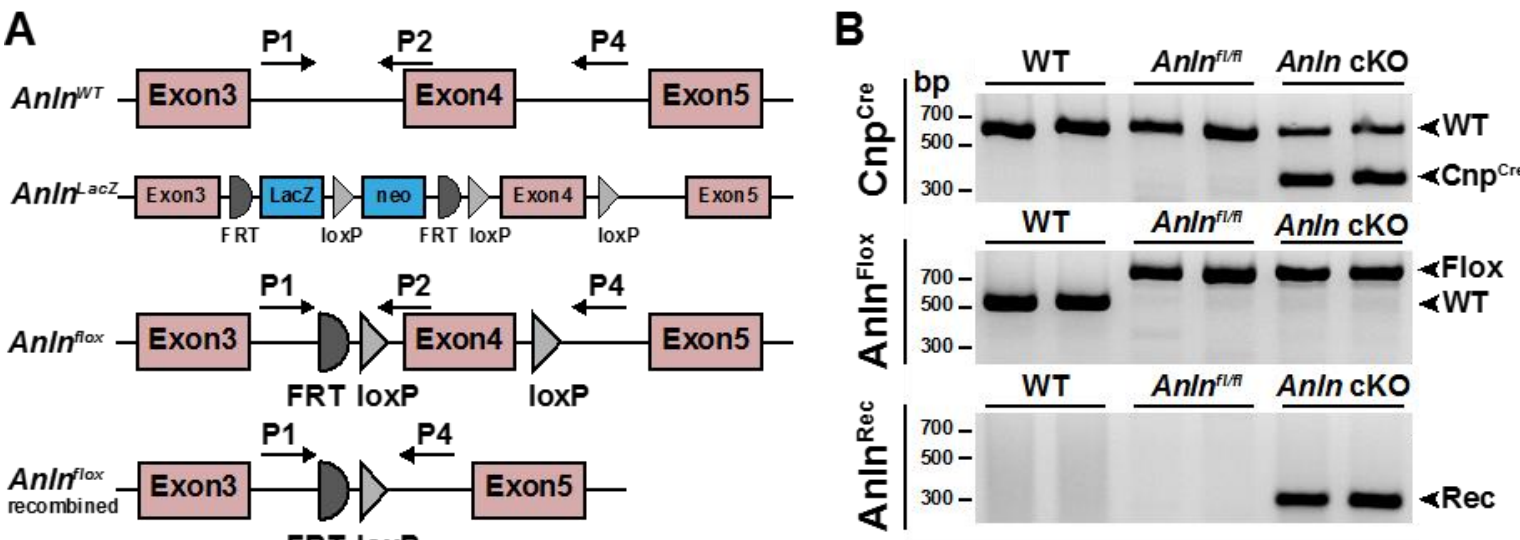

Figure 11: Generation of $\boldsymbol{A n} \mathbf{I n}^{\mathrm{f} / \mathrm{fl}} ; \boldsymbol{C n}^{\mathrm{Cre} / \mathrm{Wt}}$ mice. (A) Scheme for conditional inactivation of the Anln gene. Exon 4 of the Anln allele is flanked by loxP-sites for Cre-mediated recombination. Positions of PCR primers (P1, P2, P4) are indicated. (B) Genotyping PCR of DNA isolated from mouse tail tips at P21. Upper row depicts Crespecific PCR product in An/n cKO mice. Middle row depicts PCR product of primer P1 and P2 in controls (Wt and $\left.A n / n^{t / f / t}\right)$ and $A n / n$ cKO mice. Note that the loxP-sites increase the PCR product as expected. Lower row depicts PCR product of primer P1 and P4 after recombination. Note that the recombination band is specific for Anln cKO mice.

\subsubsection{Mass spectrometric comparison of Anln cKO mice and controls}

For quantitative analysis of protein abundances, myelin of controls and Anln cKO mice were compared using mass spectrometry (MS) (Olaf Jahn, MPI EM; proteomics unit). In total, 446 proteins were identified using a HD-MSE approach. In Figure 12A, the data for all identified proteins are plotted as $\log _{2}$ fold change $\left(\log _{2}\right.$ FC) versus the $-\log 10$ of the modified $p$-value (q-value) in a Volcano plot. The dashed lines indicate the applied threshold of +1 and $-1 \log _{2}$ FC, indicative of a halved or doubled abundance of a protein in myelin, respectively. Only CNS myelin septins are reduced below the applied threshold. CDC42 and $\mathrm{RHOB}$ are also significantly reduced regarding the q-value, but not below the fold change threshold. These proteins are also known to be involved in ANLN/septin filament formation (light red dots, Figure 12A). As ANLN is not detected by MS in myelin of Anln cKO mice (Figure 12B) it is not included in the Volcano plot (Figure 12A). However, the genotype dependent reduction of CNP is detected by MS (Figure 12B). Figure $12 \mathrm{C}$ displays the comparison between myelin of controls and Anln cKO mice of ANLN/septin filament formation related proteins (SEPT4, SEPT2, SEPT8, SEPT7, RHOB, CDC42) (Joberty et al., 2001, Renshaw et al., 2014, Mendoza et al., 2002). The proteins are sorted from highest decrease in AnIn cKO mice to lowest decrease in abundance of the 6 selected proteins. All CNS myelin septins are reduced more than 50\% ( $p$-value $<0.0001$ ), RHOB and CDC42 are also significantly reduced $(\mathrm{ROHB} p=0.0022$; $\mathrm{CDC} 42 \mathrm{p}=0.0368)$. Within the 446 identified 
proteins, classical myelin markers were selected and compared between genotypes (Figure 12D). Throughout all identified myelin marker proteins (except CNP; see above), no significant change was detectable. Since anillin is known to interact with other cytoskeletal components (Piekny and Glotzer, 2008, Straight et al., 2005, Piekny and Maddox, 2010, Versele and Thorner, 2005), all cytoskeleton related proteins within the dataset were identified and compared between the genotypes. There was no significant change in abundance throughout all identified cytoskeletal markers.

To summarize, the results support the efficient depletion of ANLN from oligodendrocytes in AnIn cKO mice. Further, the loss of ANLN in myelinating glia cells leads to a strong reduction of CNS myelin septins but has no effect on other detected proteins. These results further strengthen the expected interaction of anillin and septins in CNS myelin. 
A

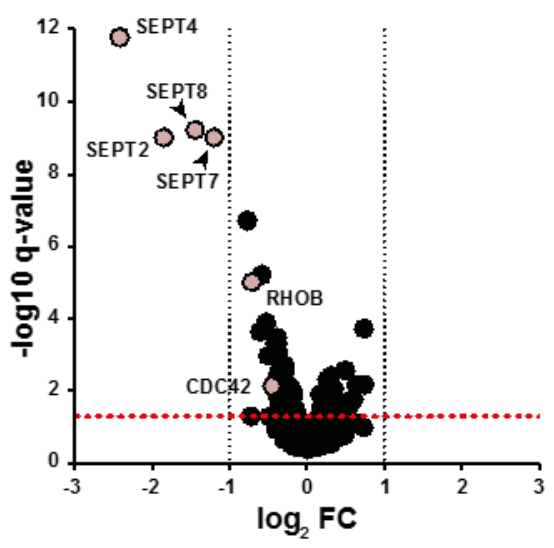

B

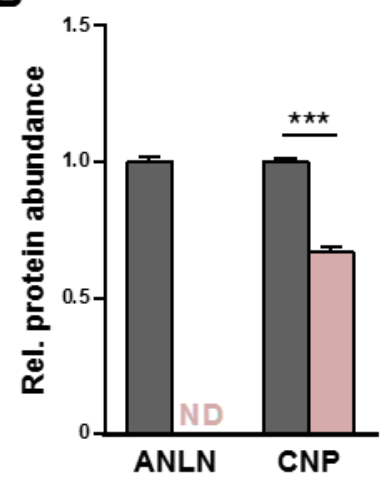

C

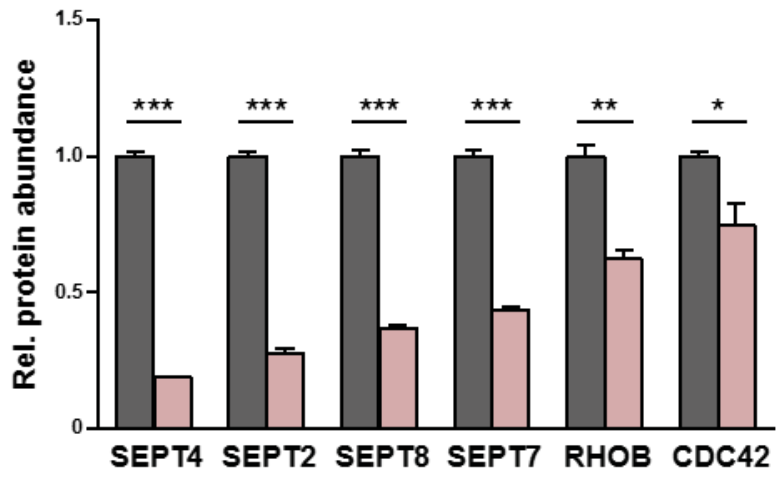

D

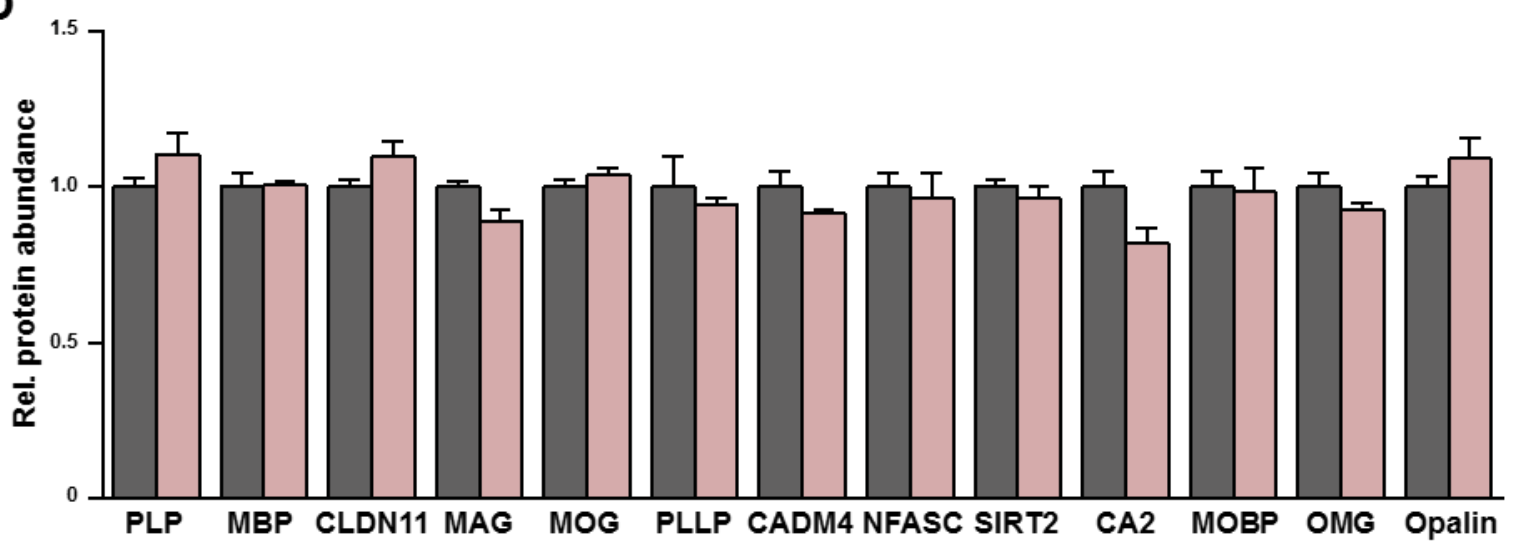

E

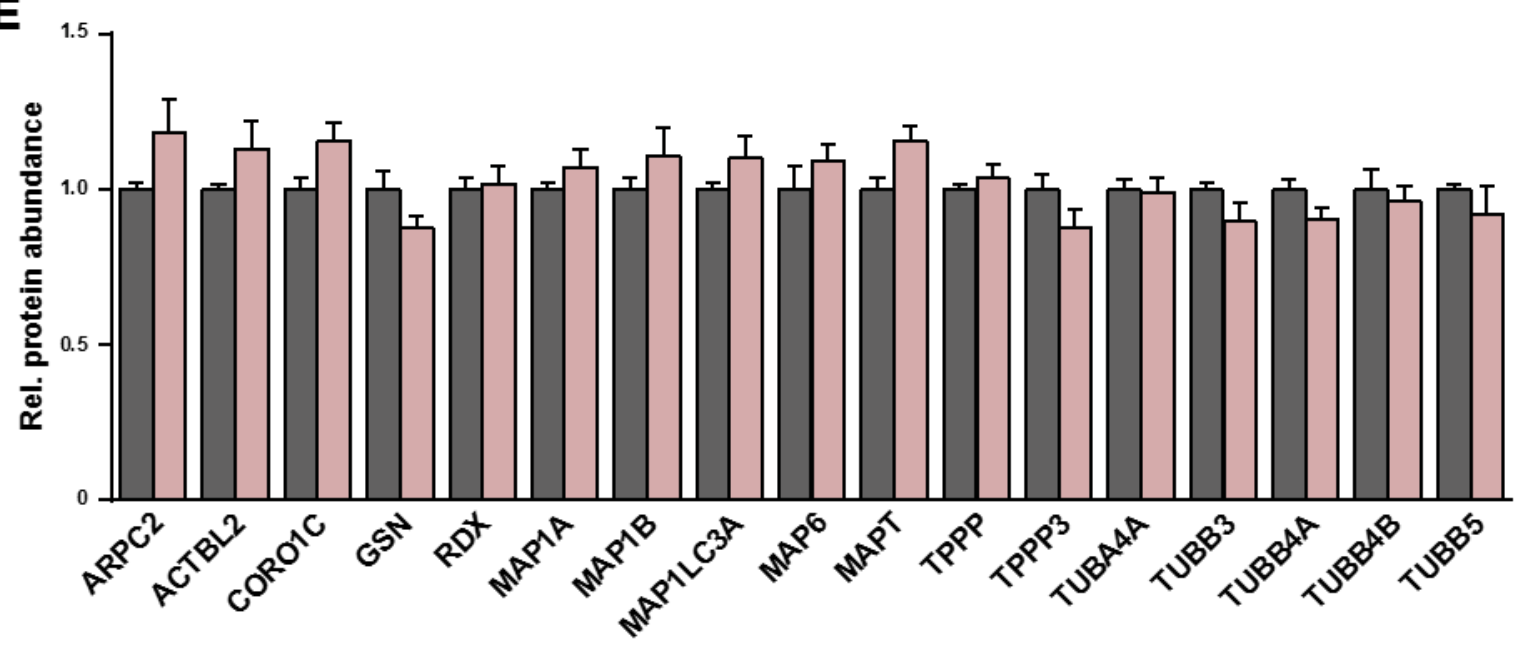


Figure 12: Mass spectrometric analysis of purified myelin. (A) Volcano plot of proteome data comparing proteins of purified myelin of $A n / n$ cKO to $A n / n^{f / f f l}$. The data for all identified proteins are plotted as $\log _{2}$ fold change $\left(\log _{2} \mathrm{FC}\right)$ versus the - $\log 10$ of the modified $p$-value (q-value). Black dashed lines indicate the applied threshold of $+/-1 \log _{2}$ FC. Red dashed line indicates a q-value of 0.05 . Proteins with relation to septin/anillin filament formation are highlighted as light red dots and labeled with protein names. It is of note that all myelin septins (SEPT2, SEPT4, SEPT7, SEPT8) are reduced in myelin of Anln cKO mice compared to An/ $n^{f / / f l}$ mice. ANLN is not depicted, as it is not detected in myelin of Anln cKO mice. (B) Differential myelin proteome analysis confirms genotype dependent expression of ANLN and CNP in myelin purified from $A n / n^{f / f l} ; C n p^{C r e / W t}$-mice $(A n l n$ cKO) at P75. Note that ANLN was not detectable. Mean +/ SEM. $n=3$ animals per genotype; two-tailed unpaired t-test CNP $p<0.0001$. (C) Differential myelin proteome analysis reveals that septins (SEPT2, SEPT4, SEPT7, SEPT8) are more than $50 \%$ diminished in myelin purified from Anln cKO mice at P75 compared to An/ $n^{f / f l}$ mice. Further, RHOB and CDC42 are significantly reduced in myelin purified from Anln cKO-mice at P75. Mean +/ SEM. $n=3$ animals per genotype; two-tailed unpaired t-test SEPT4 $p<0.0001$; SEPT2 $p<0.0001$; SEPT8 $p<0.0001$; SEPT7 $p<0.0001$; ROHB $p=0.0022$; CDC42 $p=0.0368$. (D) Differential myelin proteome analysis reveals that classical myelin proteins are not affected. Mean + / SEM. $n=3$ animals per genotype; two-tailed unpaired t-test. (E) Differential myelin proteome analysis reveals that all identified cytoskeletal proteins are not affected. Mean +/ SEM. $n=3$ animals per genotype; two-tailed unpaired t-test. Mass spectrometry was performed by Dr. Olaf Jahn and the proteomics unit of the MPI EM.

\subsubsection{Loss of myelin septins upon conditional depletion of ANLN}

To validate the results of the MS analysis, immunoblots were performed. Comparing lysate and purified myelin (Figure 13A), ANLN was only detectable in myelin and absent in Anln cKO mice. The smaller isoforms of SEPT8 are enriched in the myelin fraction and reduced in abundance in myelin of Anln cKO mice. MOG is loaded as a control for the myelin fraction and therefore, shows an increase in abundance in the myelin fraction. TUJ1, as an axonal marker, is strongly reduced in the myelin fraction. The abundance of CNS myelin septins was then further analyzed on purified myelin (Figure 13B). As expected, ANLN was not detectable in Anln cKO mice. Furthermore, all CNS myelin septins (SEPT2, SEPT4, SEPT7, SEPT8) were strongly reduced in abundance. ATP1A3 was loaded as control and exhibited no difference in abundance between controls ( $\left.A n / n^{n / / / h}\right)$ and Anln cKO mice. In a next step, classical myelin proteins were analyzed (Figure 13C). AnIn cKO mice display a genotype dependent reduction of CNP of about $50 \%$. All other tested myelin proteins (PLP, SIRT2, CD9, CA2) were unchanged. ATP1A1 was loaded as control and displayed no difference in abundance between controls $\left(A n / n^{f / f}\right)$ and $A n / n$ cKO mice.

Taken together, the results validate the data of the MS analysis. The genotype dependent depletion of ANLN is functional and results in a strong reduction of septins within the myelin fraction, whereas the abundance of myelin proteins is not affected. 


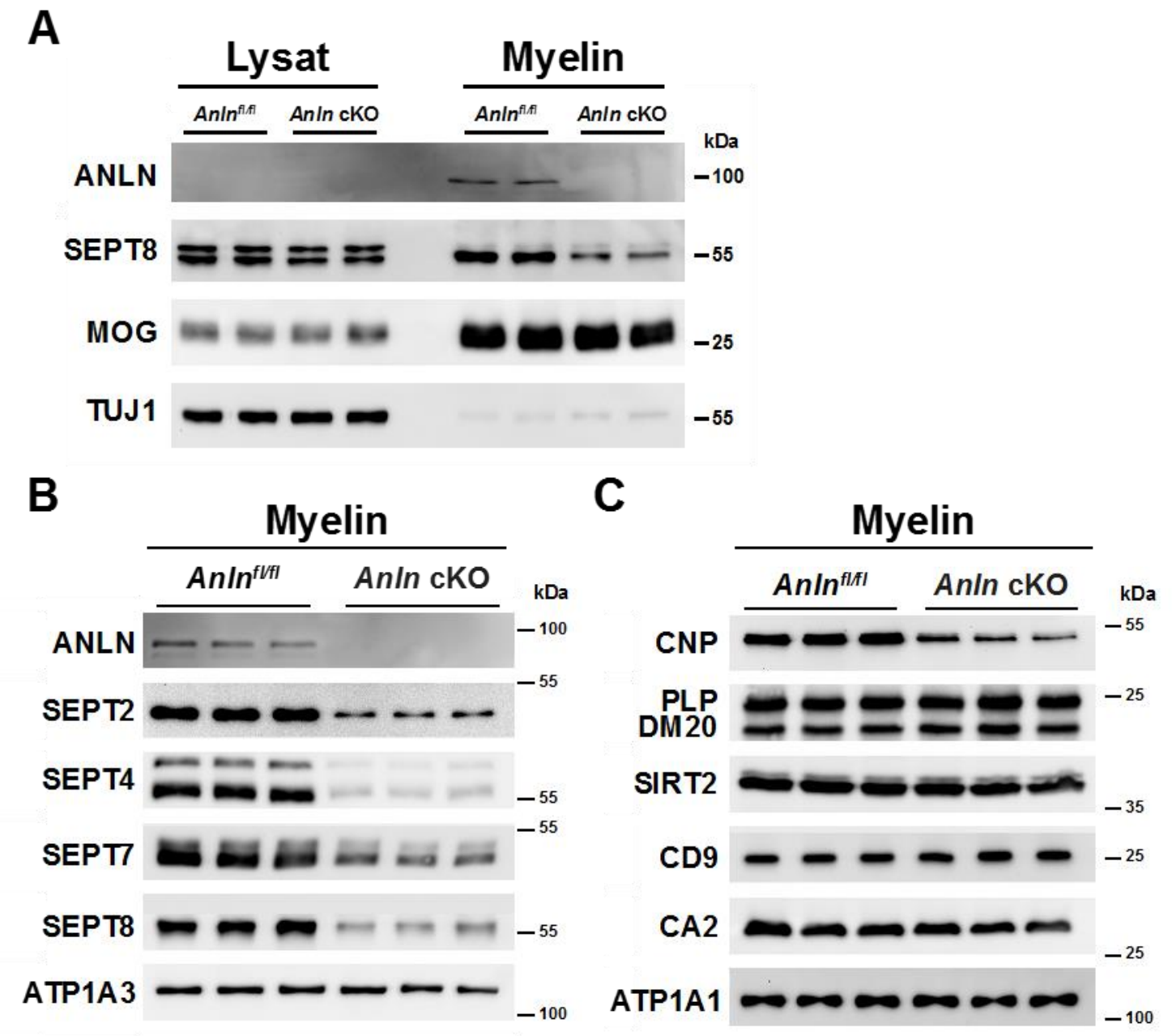

Figure 13: Abundance of ANLN, septins, and classical myelin proteins. (A) Immunoblot analysis of brain lysates and myelin purified from P75 control mice $\left(A n / n^{f / / f}\right)$. ANLN is just detectable in purified myelin. MOG and TUJ1 were detected as controls. (B) Immunoblotting validates the lack of anillin (ANLN) and the strong reduction of myelin septins (SEPT2, SEPT4, SEPT7, SEPT8) in myelin purified from brains of Anln cKO mice. ATPase $\mathrm{Na}+/ \mathrm{K}+$ transporting subunit alpha 3 (ATP1A3) was detected as a control. (C) Immunoblotting validates that classical myelin proteins are not affected in myelin purified from brains of Anln cKO mice. Of note is that CNP is reduced, genotype dependent, about $50 \%$. ATPase $\mathrm{Na}+\mathrm{K}+$ transporting subunit alpha 1 (ATP1A1) was detected as a control.

\subsection{4 mRNA abundance of regulated proteins}

To analyze whether proteins that were found to be downregulated on immunoblot level and/or downregulated within the MS data are also regulated on gene level or posttranscriptional, mRNA abundances were quantified using qRT-PCR (Figure 14). To do so, corpus callosi of control and Anln cKO (male and female) mice were dissected ( $\mathrm{n}=6$ per genotype) and analyzed. The quantification shows that the mRNAs of $A n l n(>98 \%)$ and Cnp ( $50 \%)$ are downregulated depending on the genotype. Other quantified mRNAs (Sept2, Sept4, Sept7, Sept8, RhoB, Cdc42) were not altered, indicating that the reduction on protein level of these genes was due to post-transcriptional modifications. qRT PCRs were performed by Ursula Kutzke (MPI EM). 


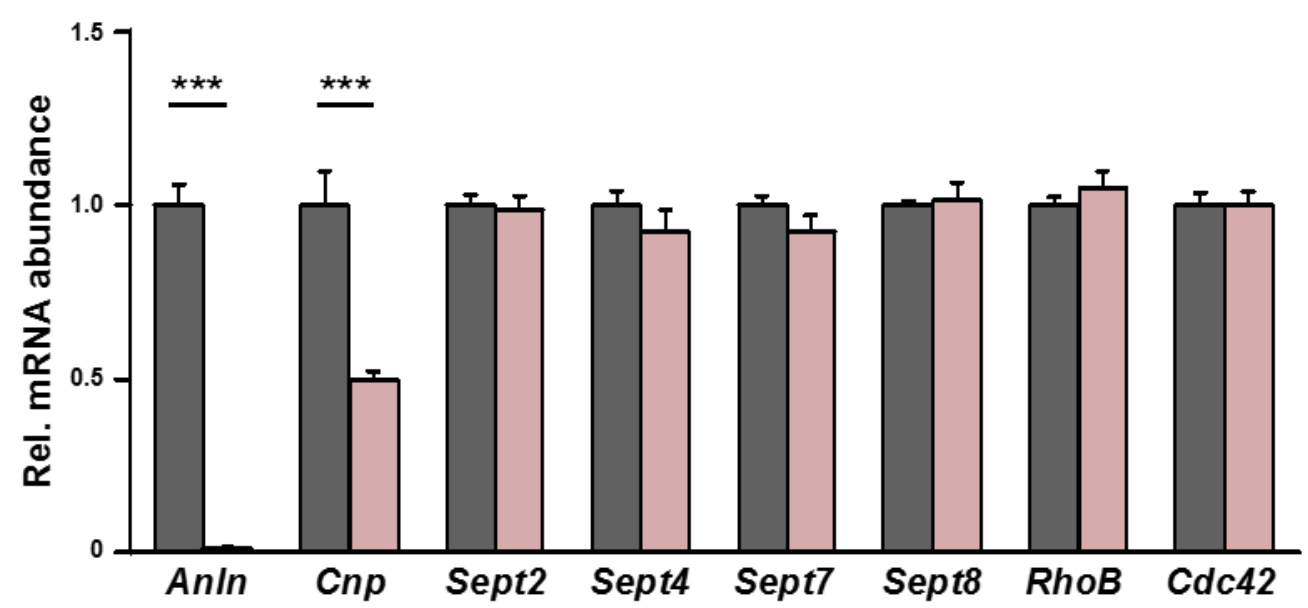

Figure 14: qRT-PCR analysis of regulated proteins. qRT-PCR to determine the abundance of Anln, myelin septins, $R h o B$, and $C d c 42$ mRNAs in white matter (corpus callosum) of control $\left(A n / n^{f / f f}\right)$ and Anln cKO mice. Anln and Cnp mRNA is significantly downregulated as expected due to the genotype. Note that myelin septins and $C d c 42$ mRNAs were unaltered in abundance in Anln cKO mice compared to control mice $\left(A n / n^{f / f t}\right)$. Mean $+/$ SEM. $\mathrm{n}=6$ animals per genotype; two-way ANOVA; Anln $\mathrm{p}<0.0001$; Cnp $\mathrm{p}<0.0001$. qRT PCRs performed by Ursula Kutzke (MPI EM).

\subsubsection{Myelin abnormalities}

As it was previously shown that lack of the septin cytoskeleton in oligodendrocytes cause myelin outfoldings (Patzig et al., 2016), it was analyzed whether the detected reduction of ANLN and septins in Anln cKO mice lead to pathological myelin outfoldings. For analysis, electron micrographs of optic nerve cross sections at different time points (P14, P75, 6 mo, $8 \mathrm{mo}$ ) were analyzed. Figure 15A illustrates examples of myelin outfoldings in both, controls and Anln cKO mice, highlighted by dashed lines. The images already indicate that outfoldings in Anln cKO mice are more severe than in controls. The quantification (Figure $15 B)$ reveals, that from P75 on, the \% of area covered by myelin outfoldings is significantly higher in Anln cKO mice compared to controls. The area increases with age, suggesting a worsening of the phenotype.

To summarize, the results indicate that the loss of ANLN/septin filaments lead to the formation of myelin outfoldings.

$A n I n^{f / f l} \quad \square A n I n$ cKO

A

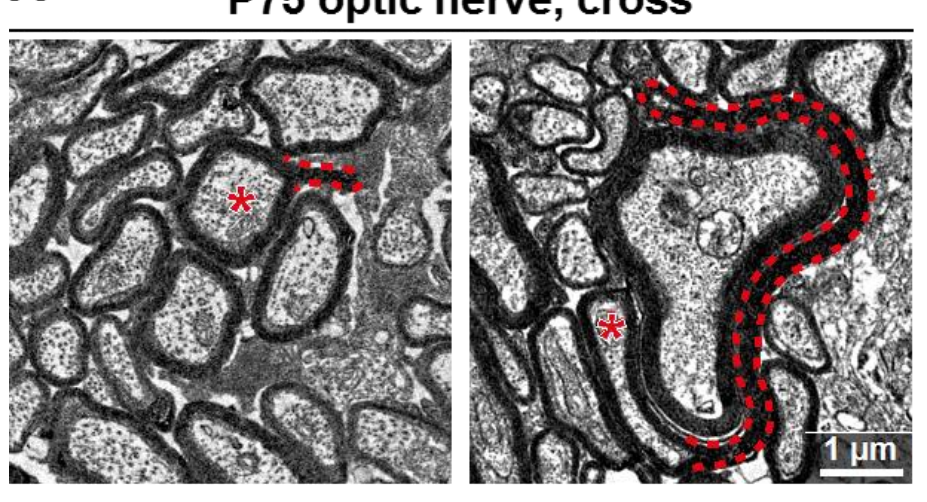

B

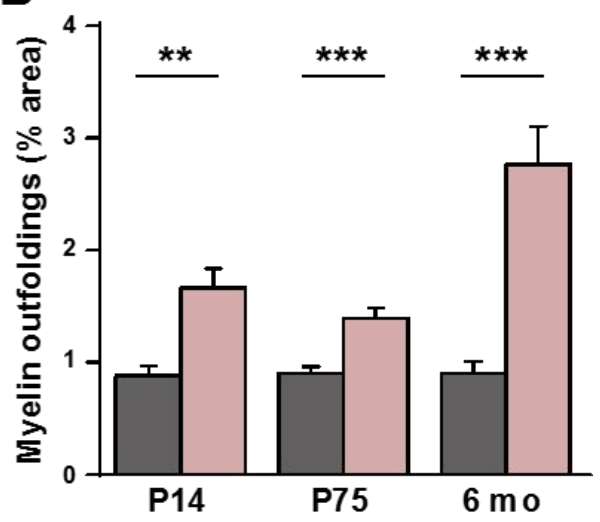


Figure 15: Lack of ANLN/septin filament causes myelin outfoldings. (A) Electron micrographs of optic nerves exemplify myelin outfoldings at P75. Dashed lines highlight myelin outfoldings; associated axons are marked with asterisks. (B) Quantitative evaluation of optic nerve electron micrographs reveals progressive myelin outfoldings in adult $A n I n^{f / f l} ; C n p^{C r e / W t}$ mice (Anln cKO). Mean +/ SEM. $\mathrm{n}=4-6$ animals per condition; twotailed unpaired t-test P14 $\mathrm{p}=0.0076 ; \mathrm{P} 75 \mathrm{p}=0.0009 ; 6 \mathrm{mo} \mathrm{p}=0.0007$.

\subsubsection{Structure of myelin outfoldings}

To get an overview of the structure and the size of myelin outfoldings, a 3D reconstruction of this pathological phenotype was generated from optic nerves of Anln cKO mice and respective controls at 5.5 mo of age by using FIB SEM (see also materials and methods section 3.2.9, performed by Dr. Anna Steyer (EM facility; MPI EM)). Figure 16A and 16A' illustrate 3D reconstructions of an $A n / n^{t / / f}$ optic nerve. Figure $16 \mathrm{~A}$ depicts the reconstruction of one axon (blue), whereas A' also depicts the myelin (yellow) surrounding the axon. Figure $16 \mathrm{~B}$ and $16 \mathrm{C}$ illustrate reconstructions of AnIn cKO optic nerves. In the column $\mathrm{B}$ only one axon, which exhibits an outfolding is depicted, whereas in column $C$ all axons exhibiting outfoldings are shown. In B' the length of the reconstructed outfolding was exemplary calculated, revealing a length of $\sim 12 \mu \mathrm{m}$. In $\mathrm{C}^{\prime}$ it can be seen that several axons exhibit myelin outfoldings and that these outfoldings occupy a considerable space.

The 3D reconstructions exhibit that the quantified outfoldings are neither hairpins, nor are they present along the whole internode. Furthermore, the reconstructions illustrate that myelin outfoldings occupy a considerable space, and therefore may have further impact on the functionality of axons.

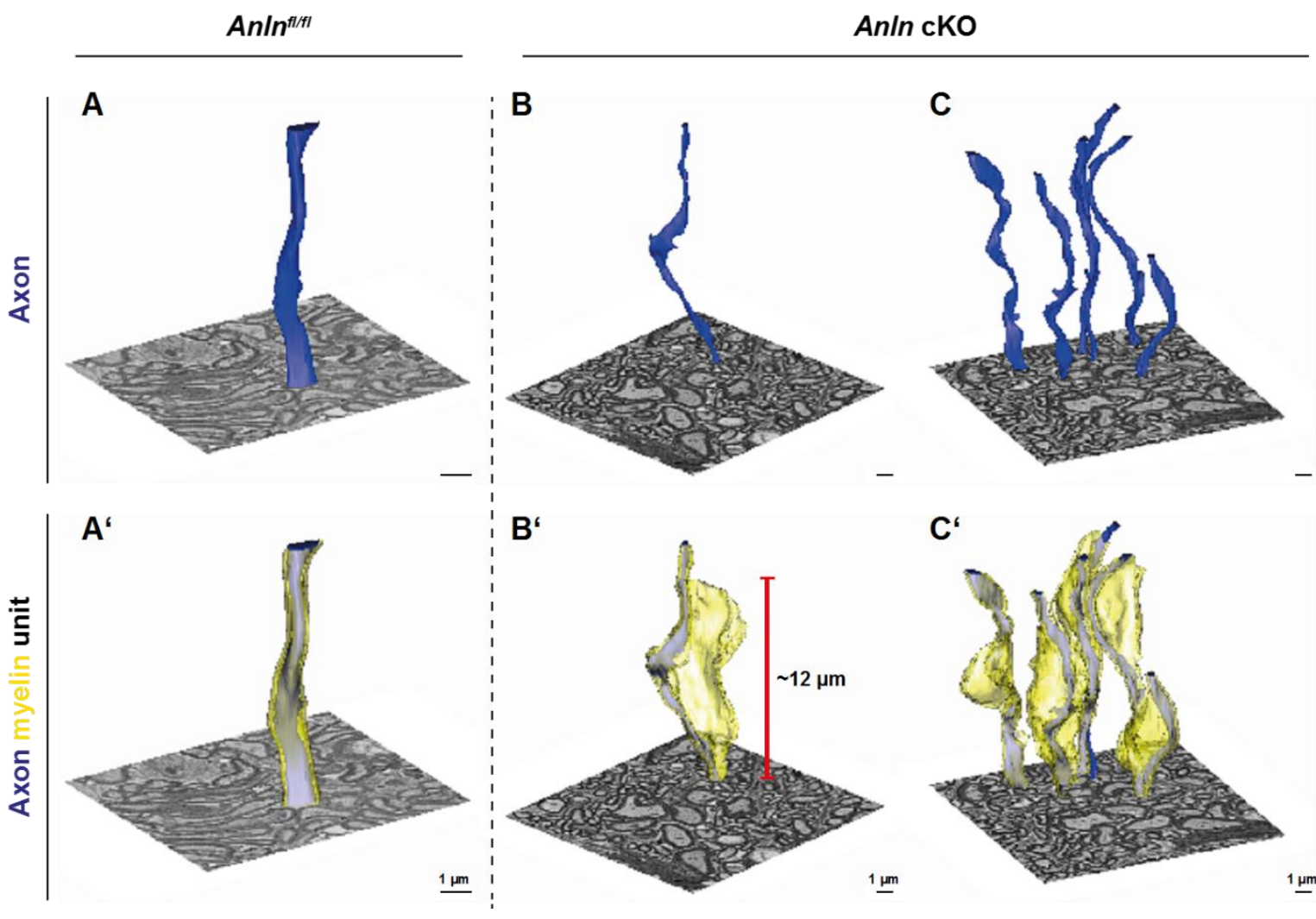


Figure 16: 3D reconstruction of myelin outfoldings. (A-C) 3D reconstructions of focused ion beam-scanning electron microscopy (FIB-SEM) micrographs displaying the plasma membrane of myelinated axonal segments (blue) and respective myelin sheaths (yellow) in the optic nerve of control $\left(A n / n^{f / f}\right)\left(\mathbf{A}, \mathbf{A}^{\prime}\right)$ and $A n l n$ cKO $\left(\mathbf{B}, \mathbf{B}^{\star}\right.$, C, $\left.\mathbf{C}^{\prime}\right)$ mice at $\sim 5.5 \mathrm{mo}$. The myelin sheath reconstructed in $\mathbf{A}^{\prime}$ is tightly associated with the corresponding axon $\left(\mathbf{A}, \mathbf{A}^{\prime}\right)$ in the control nerve. An individual myelin outfolding $\left(\mathbf{B}^{\prime}\right)$ and the corresponding axon are reconstructed in $\mathbf{B}, \mathbf{B}^{\prime}$. All myelin outfoldings in that same block (as in $\mathbf{B}, \mathbf{B}$ ) were reconstructed in $\mathbf{C}$ with their corresponding axons (in $\mathbf{C}, \mathbf{C}^{`}$ ). It is of note that myelin outfoldings represent large sheets of compacted multilayered membrane stacks that extend considerably away from their respective myelinated axon, displaying longitudinal dimension of about $12 \mu \mathrm{m}$ (indicated in B'). $\mathrm{n}=1$ for each genotype. FIB SEM reconstructions were performed by Dr. Anna Steyer.

\subsubsection{Decelerated nerve conduction velocity in Anln cKO mice}

As described above, myelin outfoldings occupy a considerable space, which might result in a disrupted insulation of axons and thereby influencing nerve conduction velocity. To analyze, whether outfoldings might have an effect on the insulation and therefore on signal propagation, in vivo nerve conduction velocity measurements at the spinal cord were performed (Dr. Payam Dibaj, MPI EM). The measurements show a significantly decelerated nerve conduction velocity of about $15 \%$ in Anln cKO mice compared to controls (Figure 17A). As changes in nerve conduction velocity can also arise due to changes in the nodal structure (Arancibia-Carcamo et al., 2017), the nodal and paranodal organization was visualized by immunohistochemistry on longitudinal sectioned spinal cord cryosections and quantified using ImageJ (Figure 17B-G). The nodal density was quantified in Figure 17B, displaying equal amounts of nodes per $\mu \mathrm{m}^{2}$ in controls and Anln cKO mice. In Figure 17C, an example of a node is depicted for each genotype. CASPR (green) is labeled to visualize the paranodes, whereas $\mathrm{Na}_{v} 1.6$ (red) is labeled to visualize the node. The images of the nodes display no structural changes in the nodal and paranodal organization in Anln cKO mice compared to controls. In the merge of the control, the measured parameters for the quantification are indicated. To quantify possible nodal changes, the nodal length was measured using ImageJ (Figure 17D). The quantification shows no difference between controls and Anln cKO mice in node length. For the paranode length quantification, both paranodes contacting a node were measured and the average was calculated for comparison of controls and Anln cKO mice. The quantification shows no difference between controls and Anln cKO mice (Figure 17E). Additionally, the ratio of node length to diameter (Figure 17F) and the ratio of paranode length to diameter (Figure 17G) were calculated and not found significantly altered between genotypes.

Accordingly, these results indicate a healthy and normal organization of the nodes, supporting the possible direct impact of myelin outfoldings on the nerve conduction velocity. 
A
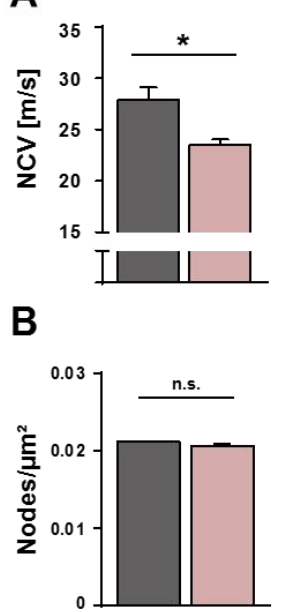

C

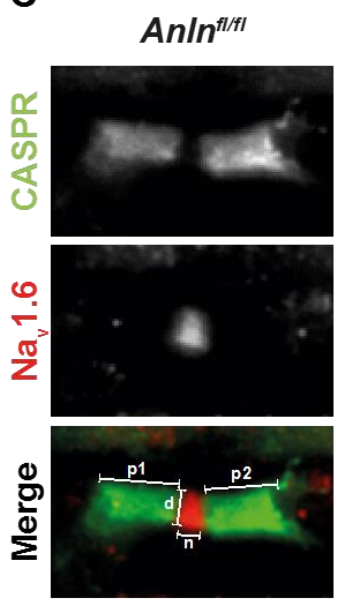

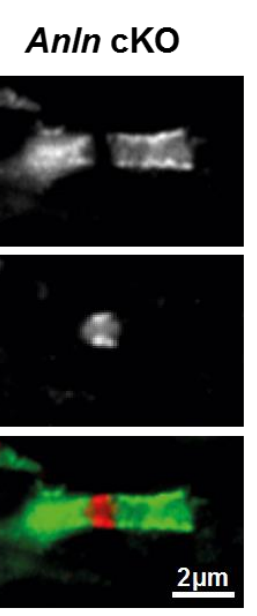

D

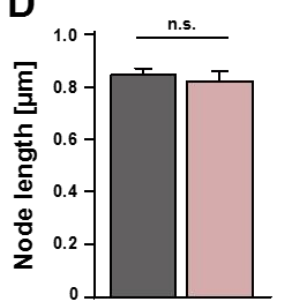

$\mathbf{F}$

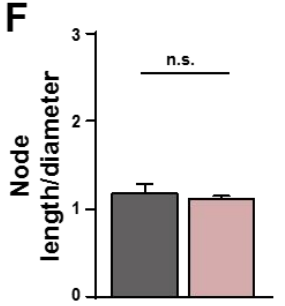

E

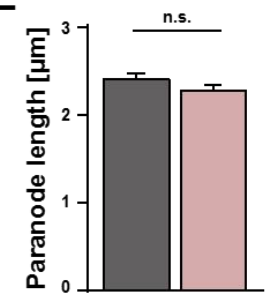

G

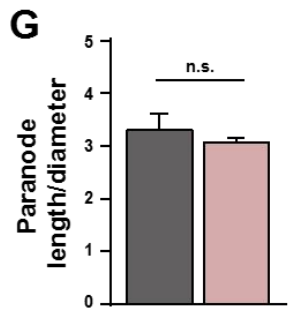

Figure 17: Nerve conduction velocity and node structure. (A) Electrophysiological measurement reveals decelerated nerve conduction in spinal cords of $A n / n$ cKO mice compared to controls $\left(A n / n^{f / f}\right)$ at 6 month of age. Mean + / SEM. $n=7-11$ animals per genotype; two-tailed unpaired $t$-test $(p=0.0149)$. Nerve conduction velocity measurements were performed by Dr. Payam Dibaj (MPI EM). (B) Quantitative analysis of node density on longitudinal spinal cord sections of control $\left(A n / n^{f / / f}\right)$ and $A n / n$ cKO mice at P75. Density of nodes is unchanged; not significant (n.s.) according to unpaired two-tailed t-test; $n=4$ animals per genotype; $p=0.1985$. (C) Immunohistochemical detection of a marker of the nodes of Ranvier ( $\mathrm{Nav}_{\mathrm{v}} 1.6$; sodium channels, red) and a paranodal marker (CASPR, green) on longitudinal spinal cord sections of control $\left(A n / n^{t / t / 1}\right)$ and Anln cKO mice at P75. Merge of control depicts measured parameters for D-G. Note that the node organization is well preserved. $\mathrm{p} 1=$ paranode length1; $\mathrm{p} 2=$ paranode length2; $\mathrm{d}=$ diameter; $\mathrm{n}=$ node length. (D) Quantitative analysis of node length indicates a normal node structure control (Anl $\left.n^{t / / t}\right)$ and Anln cKO mice at P75. Not significant (n.s.) according to unpaired two-tailed t-test; $n=4-5$ animals per genotype; $p=0.6324$. (E) Quantitative analysis of paranode length indicates a normal paranode structure in control $\left(A n l n^{t / 1 / f}\right)$ and Anln cKO mice at P75. Not significant (n.s.) according to unpaired two-tailed t-test; $n=4-5$ animals per genotype; $p=0.1927$. (F) Quantitative analysis of the ratio of node length and diameter shows no difference between control $\left(A n / n^{f / f t}\right)$ and Anln cKO mice at P75. Not significant (n.s.) according to unpaired two-tailed t-test; $\mathrm{n}=4-5$ animals per genotype; $\mathrm{p}=0.5966$. (G) Quantitative analysis of the ratio of paranode length and diameter shows no difference between control $\left(A n / n^{t / / t}\right)$ and $A n / n$ cKO mice at P75. Not significant (n.s.) according to unpaired two-tailed t-test; $n=4-5$ animals per genotype; $p=0.4603$.

\subsubsection{Unaltered brain structure and myelination in Anln cKO mice}

Since myelin proteins within the MS data remained unchanged, the brain structure and overall myelination were analyzed. On paraffin embedded coronal brain sections (Figure 18A) and sagittal cerebellum sections (Figure 18A'), the overall myelination was visualized using Gallyas silver impregnation (performed by Annette Fahrenholz). In Figure $18 \mathrm{~A}$ and $\mathrm{A}^{\prime}$ it can be seen that the brain structure is unchanged between controls and AnIn cKO mice. Furthermore, there are no changes in the overall myelination. To closer analyze the myelin ultrastructure, electron micrographs of control and AnIn cKO optic nerve cross sections were compared (Figure 18B). Each image illustrates an axon and the corresponding compact myelin layers. The black lines indicate no difference in myelin periodicity between genotypes. 
Taken together, the results indicate that oligodendrocytes, which lack ANLN, are capable of forming functional myelin.

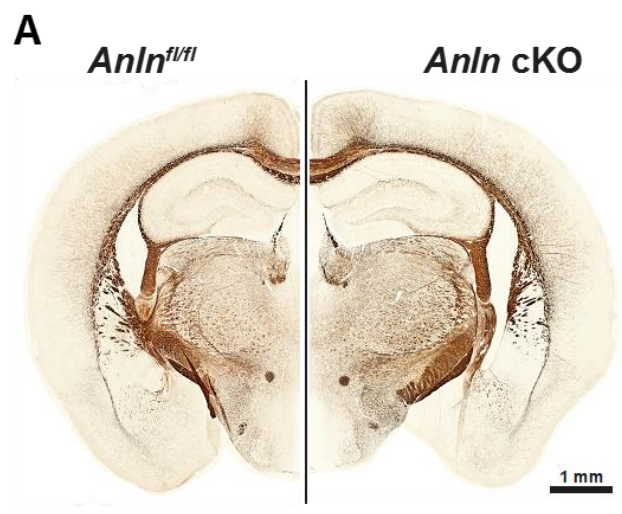

$A^{\prime}$

$A n I n^{\pi / f I}$

Anln cKO

B

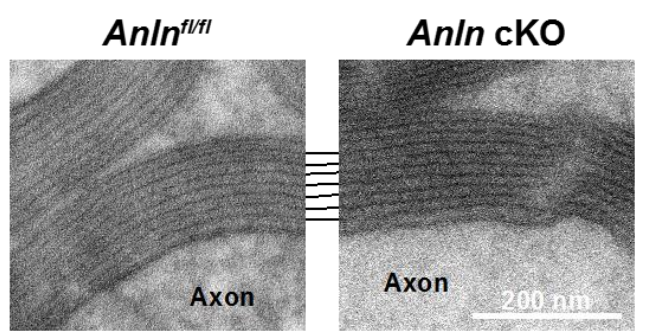

Figure 18: CNS myelination and myelin periodicity. (A-A') Silver impregnation visualizes myelinated fiber

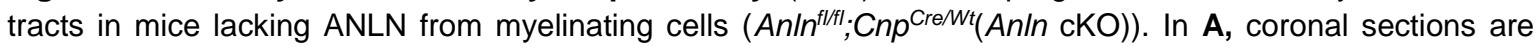
shown, whereas $\mathbf{A}^{\prime}$ illustrates sagittal sections through the cerebellum. There are no changes in myelination in Anln cKO mice at P75 compared to control mice $\left(A n / n^{t / f / t}\right)$. Images are representative of three animals per genotype. (B) Electron micrographs of 8 month optic nerves display normal myelin periodicity and compaction in $A n / n^{t / f t}$ and $A n / n$ cKO mice. The two respective axons are indicated (Axon). Images are representative of three animals per genotype.

\subsubsection{The loss of ANLN has no impact on myelin thickness}

To further assess the ultrastructure of myelin in Anln cKO mice, the g-ratio was calculated. In this calculation, the Feret axonal diameter is divided by the Feret myelin diameter to assess the ratio of axon to myelin (Figure 19A; see also 3.2.8.6). The g-ratio analysis of axons in 6 mo optic nerve cross sections shows normal values in optic nerves of control animals as well as in Anln cKO mice (Figure 19A'), revealing normal myelin thickness throughout all axon diameters. In Figure 19A" every single g-ratio of each measured axon is depicted. It can be seen that there is no difference between genotypes.

To quantify the number of myelinated axons and percentage of degenerated/degenerating axons, electron micrographs of 6 mo optic nerve cross sections were analyzed. Figure 19B displays the mean number of myelinated axons per field (field $=220 \mu \mathrm{m}^{2}$ ). The analysis depicts, that there is no difference in the number of myelinated axons between genotypes. The percentage of degenerated/degenerating profiles (Figure 19C) also exhibits no difference between genotypes. 
The results indicate that oligodendrocytes lacking ANLN are able to myelinate axons in a normal state and that the lack of ANLN in oligodendrocytes has no effect on the integrity of axons.

$A n I^{f / f t} \quad \square A n l n$ cKO

A

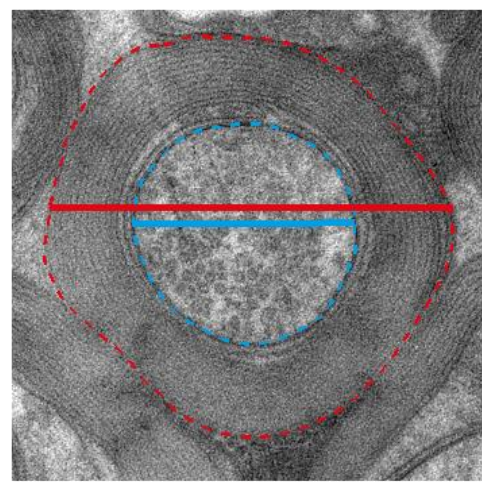

$\frac{\text { Axon diameter }}{\text { Myelin diameter }}=$ g-ratio

A“

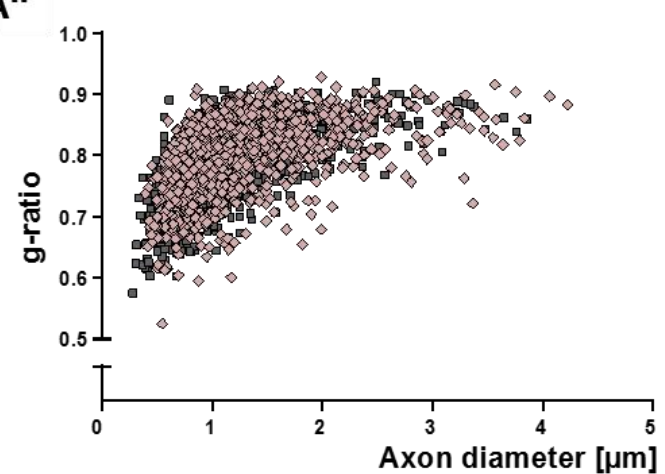

$A^{6}$

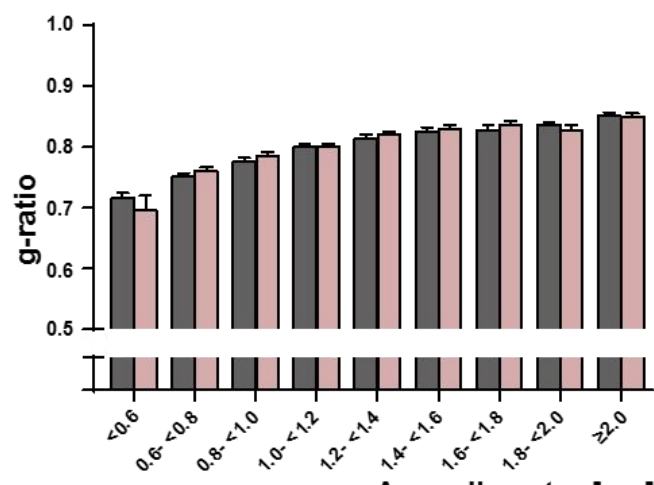

Axon diameter $[\mu \mathrm{m}]$

B

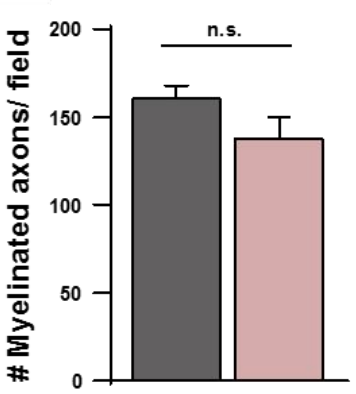

C

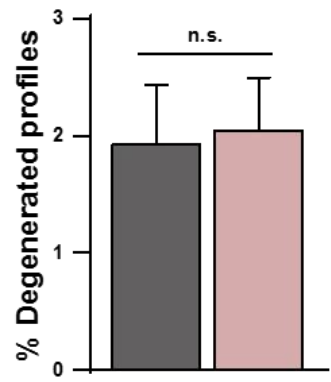

Figure 19: g-ratio and axon integrity. (A) Electron micrograph with schematic lines indicating the circumference (dashed line) and diameter (continuous line). Scheme illustrates the diameter of the axon (blue continuous line) and the diameter of the corresponding myelin (red continuous line), which are taken to calculate the g-ratio. (A'-A') g-ratio analysis of electron micrographs of optic nerves at 6 mo indicates normal myelin sheath thickness in Anln cKO mice. Mean +/ SEM. Not significant according to two-way ANOVA $(p=0.9279)$. (B) Quantitative evaluation of electron micrographs of optic nerves at 6 mo reveals a normal frequency of myelinated axons in Anln cKO mice. Mean +/ SEM. $n=4-5$ animals per genotype; not significant (n.s.) according to two-tailed unpaired t-test ( $\mathrm{p}=0.1827)$. (C) Quantitative evaluation of electron micrographs of optic nerves at 6 mo indicates the absence in Anln cKO mice of other myelin-related pathology such as degenerated axons. Mean +/ SEM. $n=4-5$ animals per genotype; not significant (n.s.) according to two-tailed unpaired t-test $(p=$ 0.8664).

\subsubsection{No signs of secondary neuropathology in CNS white matter}

As mice, which lack ANLN from oligodendrocytes, develop myelin outfoldings and display decelerated nerve conduction velocity, it was analyzed if these alterations also affect the health of axons and other brain tissue. For this purpose, mice were perfused at P75 and coronal brain sections were labeled with neuropathological markers (labeling performed by Annette Fahrenholz). The analysis was performed in the fimbria, as described in the materials and methods section 3.2.7.7. In healthy conditions, amyloid beta precursor protein 
(APP) is expressed in neurons and then further transported along the axon (Zheng and Koo, 2006). In pathological conditions of the axon, APP can accumulate and thereby indicates axonal degeneration (Coleman, 2005; Saxena and Caroni, 2007). In Figure 20A, the quantification of APP positive spheroids is shown as well as example images depicting a close up of the fimbria. Arrowheads indicate APP positive spheroids. There was no difference detectable between controls and AnIn cKO mice. In pathological conditions of brains, it is also seen that astrocytes get activated microglia-dependent, as well as the other way around (Liddelow, 2017; Jo, 2017; Skripuletz, 2013). To test for possible astrogliosis, coronal brain sections were labeled with a glial fibrillary acidic protein (GFAP) antibody and the positive area was analyzed (Figure 20B). Figure 20B depicts respective images of the staining (GFAP) in the fimbria. It can be seen that there is no difference between control and Anln cKO mice at P75. Additional to astrogliosis, microgliosis is a commonly seen phenotype in pathological brains. Microglia react to changes in brain tissue and are able to phagocytose myelin debris (Cuzner, 1988; Mosley and Cuzner, 1996). Activated microglia that changed their morphology into amoeboid cells express the surface antigen MAC3 (Ho and Springer, 1983; Giulian and Baker, 1986). To assess the area covered by activated microglia, coronal brain sections were labeled with a MAC3 antibody (Figure 20C). There were no signs of increased numbers of activated microglia in Anln cKO mice compared to controls. Further, all microglia were labeled with an antibody against IBA1 (Figure 20D). Although the area positive for IBA1 was slightly increased in AnIn cKO mice, the difference was not significant.

To sum up, the results show no signs for axonopathy, astrogliosis, and microgliosis, when oligodendrocytes lack ANLN. These observations indicate, that the loss of the ANLN/septin filament, coinciding with myelin outfoldings and decelerated nerve conduction velocity, do not cause further neuropathological abnormalities. 


\section{$A n / n^{f / f l} \quad \square$ AnIn cKO}
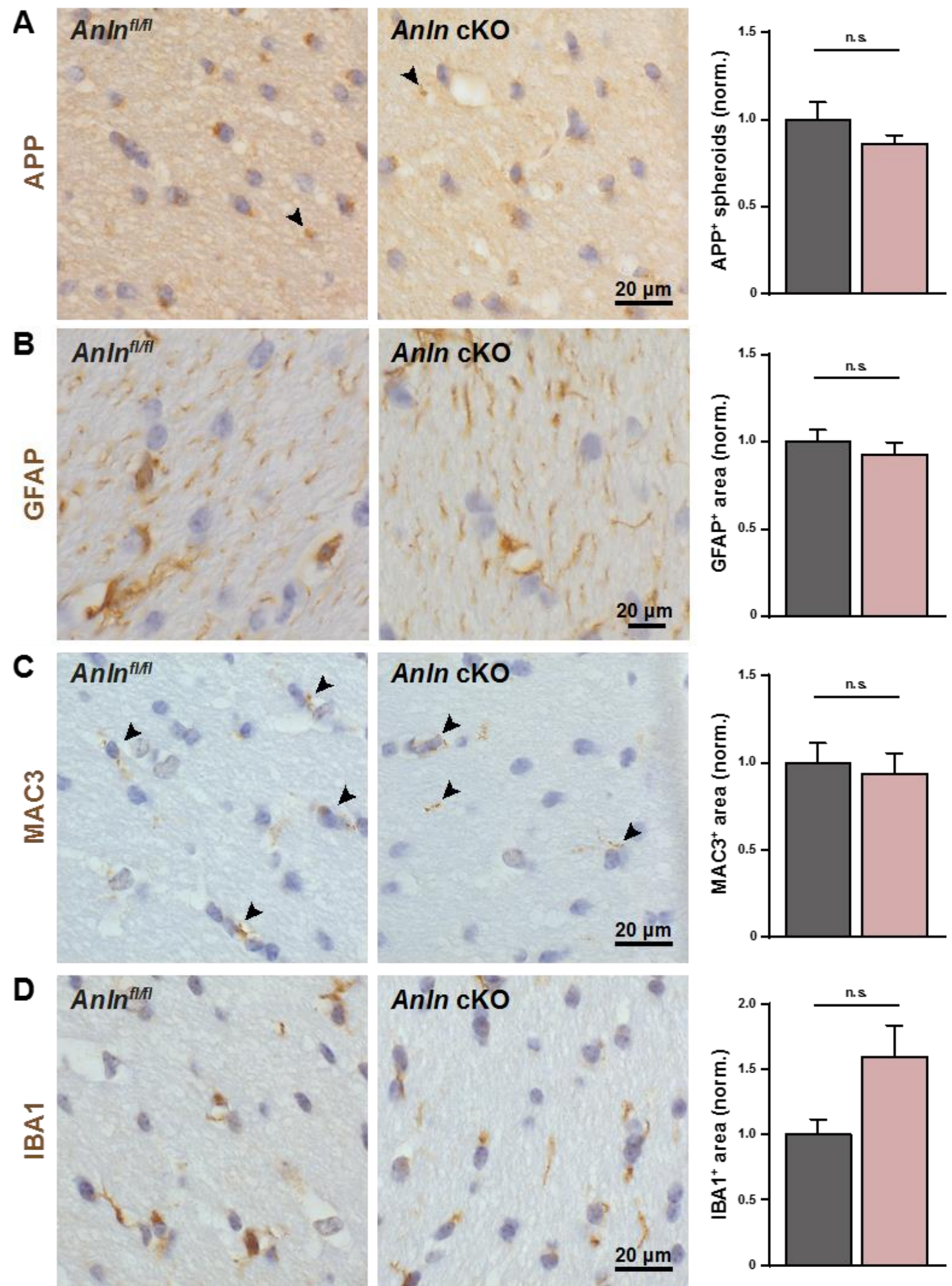

Figure 20: Lack of ANLN in oligodendrocytes does not lead to secondary neuropathology. (A) Immunohistochemical analysis of APP positive spheroids in the white matter (hippocampal fimbria) of control $\left(A n / n^{t / / f t}\right)$ and $A n / n^{f / t / t} ; C n p^{C r e W t}$ mice (Anln cKO) at P75. The labeling is similar in control and Anln cKO mice. Not significant (n.s.) according to unpaired two-tailed t-test; $n=5$ animals per genotype; $p=0.2418$. (B) Immunohistochemical analysis of GFAP-immunopositivity in the white matter (hippocampal fimbria) of control 
$\left(A n l n^{f l / f}\right)$ and Anln cKO mice at P75. The labeling is similar in control and Anln cKO mice. Not significant (n.s.) according to unpaired two-tailed t-test; $n=5$ animals per genotype; $p=0.4704$. (C) Immunohistochemical analysis of MAC3-immunopositivity in the white matter (hippocampal fimbria) of control $\left(A n / n^{f / f f}\right)$ and Anln cKO mice at P75. The labeling is similar in control and Anln cKO mice. Not significant (n.s.) according to unpaired two-tailed t-test; $n=5$ animals per genotype; $p=0.7047$. (D) Immunohistochemical analysis of IBA1-immunopositivity in the white matter (hippocampal fimbria) of control $\left(A n / n^{f / f}\right)$ and AnlncKO mice at P75. The labeling is similar in control and Anln cKO mice. Not significant (n.s.) according to unpaired two-tailed t-test; $\mathrm{n}=5$ animals per genotype; $p=0.0593$.

\subsubsection{AnIn cKO mice display reduced $\mathrm{PIP}_{2}$ levels}

It is known that ANLN is able to bind $\mathrm{PIP}_{2}$ via a $\mathrm{PH}$ (pleckstrin homology) domain at the Cterminal. Furthermore, it was shown that binding of ANLN to $\mathrm{PIP}_{2}$ promotes septin filament assembly in vitro (Kinoshita et al., 2002, Liu et al., 2012). To see, if the loss of ANLN in oligodendrocytes also affects the $\mathrm{PIP}_{2}$ levels, purified myelin was analyzed in collaboration with Prof. Dr. Ingo Heilmann (Halle, Germany). The gas chromatographic analysis reveals a significant reduction of about $50 \%$ in purified myelin of Anln cKO mice compared to controls at P75. The result suggests that ANLN may also has a stabilizing effect on $\mathrm{PIP}_{2}$.

$\square A n I n^{f f l l} \quad \square$ Anln cKO

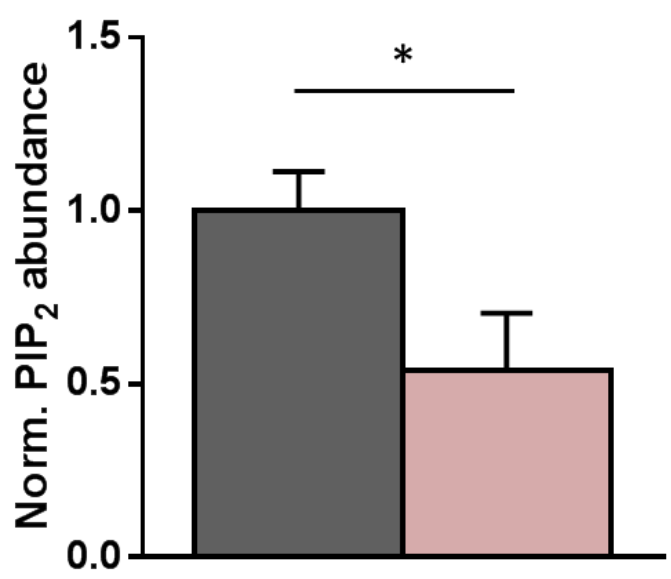

Figure 21: $\mathrm{PIP}_{2}$ analysis in AnIn cKO and control mice. Quantification of $\mathrm{PIP}_{2}$ in purified myelin. The analysis indicates decreased levels of $\mathrm{PIP}_{2}$ in Anln cKO mice compared to controls $\left(A n / n^{f / / f}\right)$ at P75. Mean $+/$ SEM. $\mathrm{n}=6$ animals per genotype; two-tailed unpaired t-test; $p=0.0435$. Gas chromatography was performed by Prof. Dr. Ingo Heilmann (Halle, Germany).

\subsubsection{Axon diameter frequency distribution}

The loss of the ANLN/septin filament leads to an instability of myelin sheaths in the CNS, resulting in myelin outfoldings. To see whether the loss of myelin stability and the altered $\mathrm{PIP}_{2}$ levels lead to axonal changes, axon diameters were analyzed at P75 and 6 mo of age in control and Anln cKO optic nerves (Figure 22). Quantification was done on EM images using Image $\mathrm{J}$ as described in the materials and methods section 3.2.8.6. Figure 22A depicts the relative frequency of indicated axon diameter at P75 in optic nerves of controls and Anln cKO mice. The bar graph indicates a trend towards larger axon diameters, but 
statistical comparison of all axon diameters with the Kolmogorov Smirnov test shows no significant difference. Since the myelin outfolding phenotype is aggravated by aging, the axon diameter frequency distribution was also analyzed at 6 mo of age (Figure 22B). The relative frequency of indicated axon diameters, again, depicts larger axon diameters in Anln cKO mice. By comparing all axon diameters, the Kolmogorov Smirnov test shows a highly significant shift towards larger axon diameters.

This result raises the possibility that the loss of the ANLN/septin filament in CNS myelin has an effect on axon diameters, suggesting that the stability of intact myelin restricts axon diameters.

For further analysis, axon diameters were also quantified in Sept8 ${ }^{\text {null/null }}$ and Sept $8^{f / f t} ; C n p^{C r e / W t}$ mice at 6 mo of age (Figure 23A and B). As these mice also lack the ANLN/septin filament, the same phenotype should be present. Figure 23A displays the relative frequency of indicated axon diameters for Sept8 $8^{\text {null/null }}$ mice. In these mice, no difference between controls and Sept8 $8^{\text {null/null }}$ mice was observed. By statistical evaluation of all axon diameters using the Kolmogorov Smirnov test, the whole distribution exhibits no significant difference. Moreover, in Sept ${ }^{f / f t /} ; C n p^{C r e / W t}$ mice the axon diameter frequency distribution was analyzed (Figure 23B). The bar graph illustrates the relative frequency of indicated axon diameters. Similar to Anln cKO mice, a shift towards larger axon diameters can be seen. Using the Kolmogorov Smirnov test, the axon diameters distribution exhibits a highly significant shift towards larger axon diameters.

$\square A n I n^{f / / f l} \square A n I n^{f / f l} ; C n p^{C r e / W T}$

\section{A P75 optic nerve}
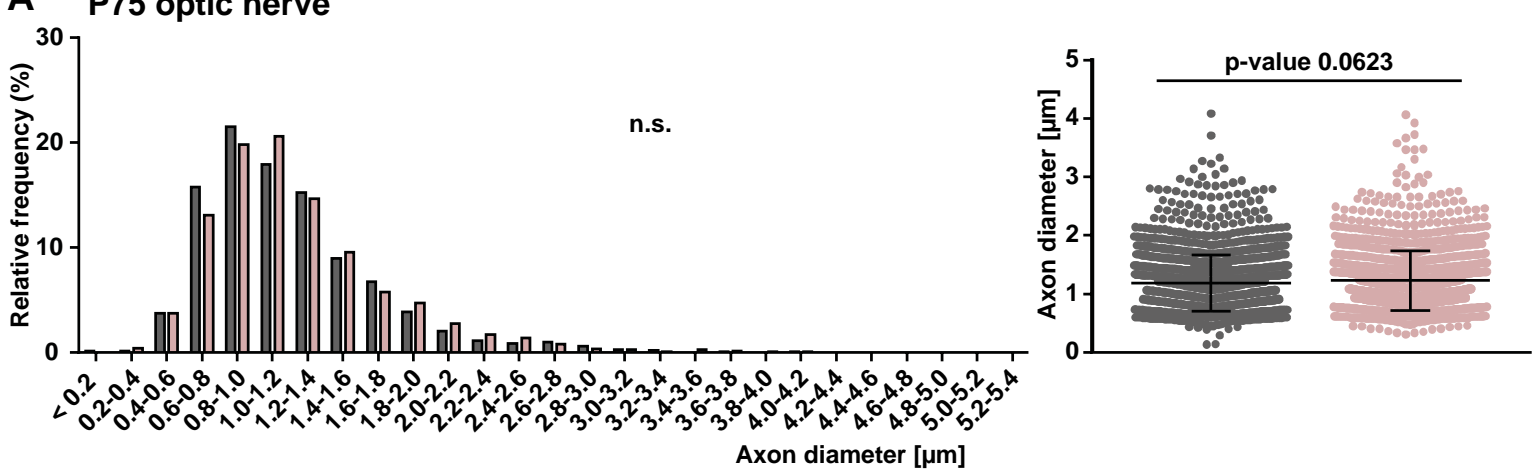

\section{B 6 mo optic nerve}
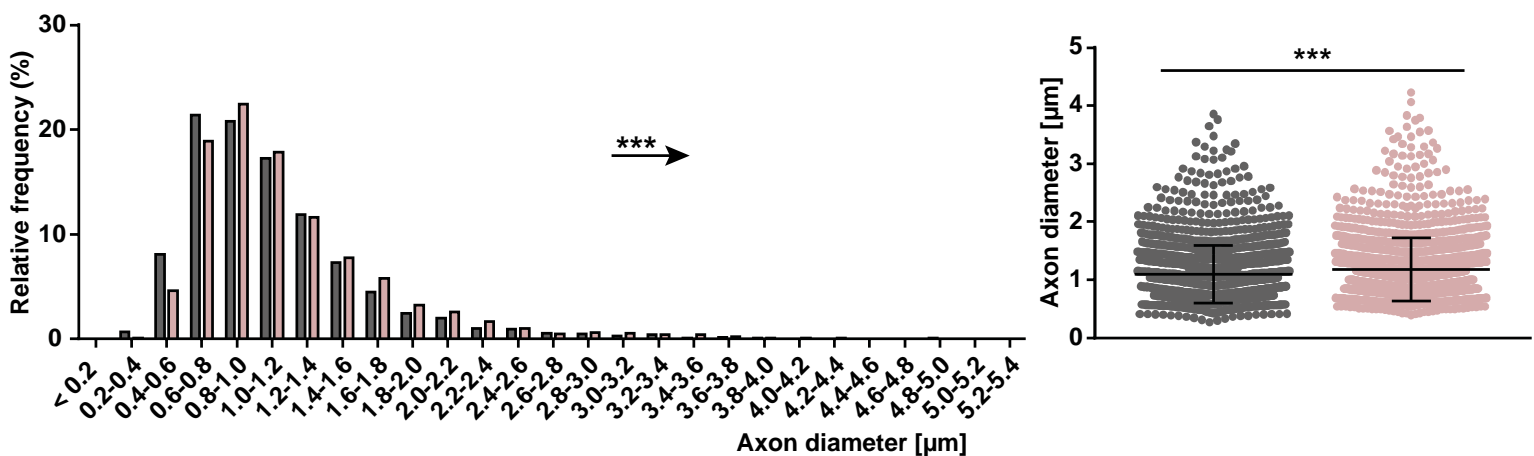

Axon diameter $[\mu \mathrm{m}]$ 
Figure 22: Axon diameter frequency distribution in AnIn cKO mice. (A) Frequency distribution analysis of optic nerve myelinated axons of electron micrographs at P75 indicates a slight trend towards larger axon diameter in Anln cKO mice. $\mathrm{n}=5$ animals per genotype. Not significant according to Kolmogorov Smirnov test $(p=0.0623)$. (B) Frequency distribution analysis of optic nerve myelinated axons of electron micrographs at 6 mo of age indicates a shift towards larger axon diameter in Anln cKO mice. $n=5$ animals per genotype. Significant according to Kolmogorov Smirnov test $(p=0.0008)$.

The results depict only a shift towards larger axon diameters in conditional mutants, which harbor half of the CNP dosage. To clarify if the observed phenotype is due to CNP heterozygosity, Cnp ${ }^{\mathrm{Cre} / \mathrm{Wt}}$ mice were analyzed at $6 \mathrm{mo}$ of age (Figure 23C). The axon diameter distribution shows no difference between controls and $C n p^{C r e / W t}$ mice, also indicated by the analysis of all axon diameters with the Kolmogorov Smirnov test.

The results presented in Figure $23 \mathrm{C}$ indicate that the lack of the ANLN/septin filament from CNS myelin possibly results in larger axon diameters, and not the CNP heterozygosity alone. Furthermore, the results display a possible new role of myelin in axon diameter restriction. 


\section{A 6 mo optic nerve}
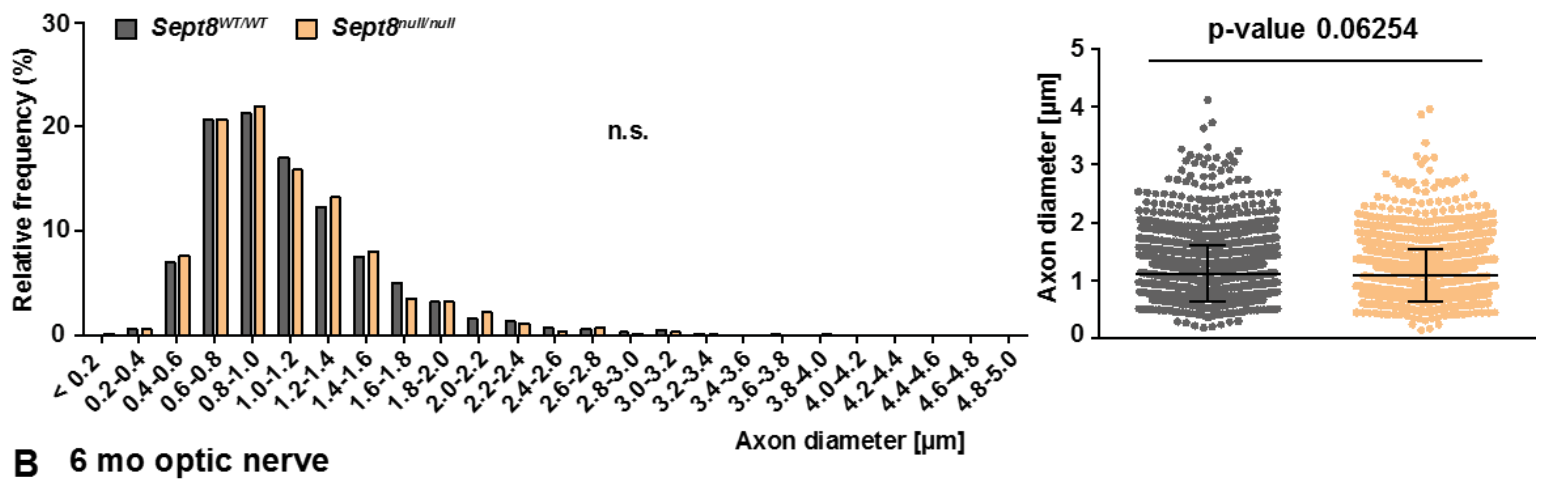

\section{B 6 mo optic nerve}

Axon diameter $[\mu \mathrm{m}]$
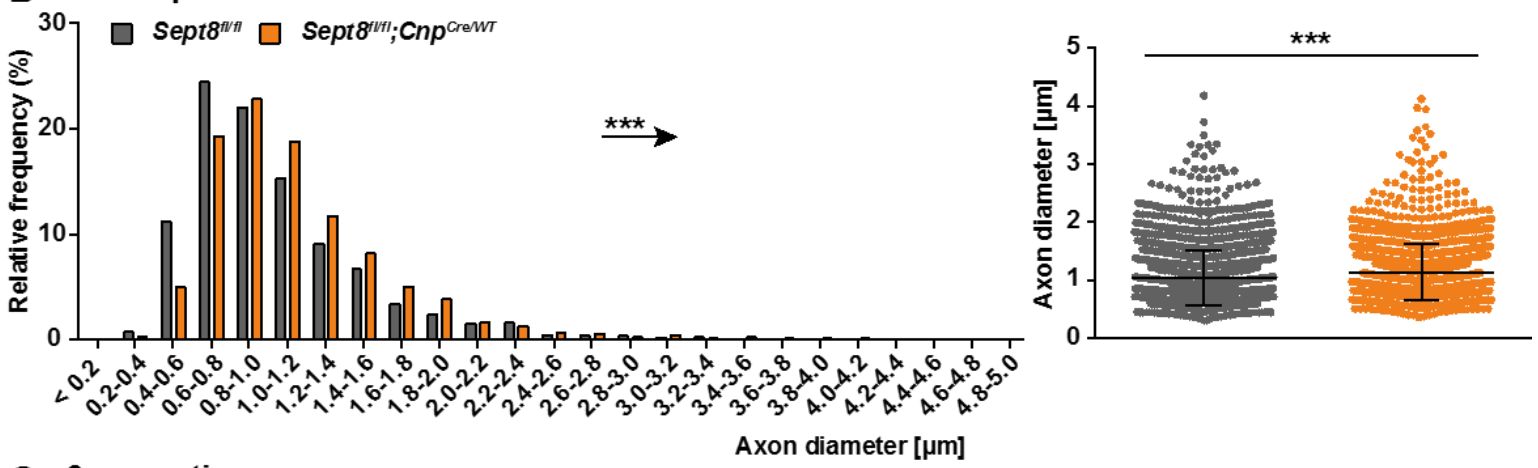

C 6 mo optic nerve
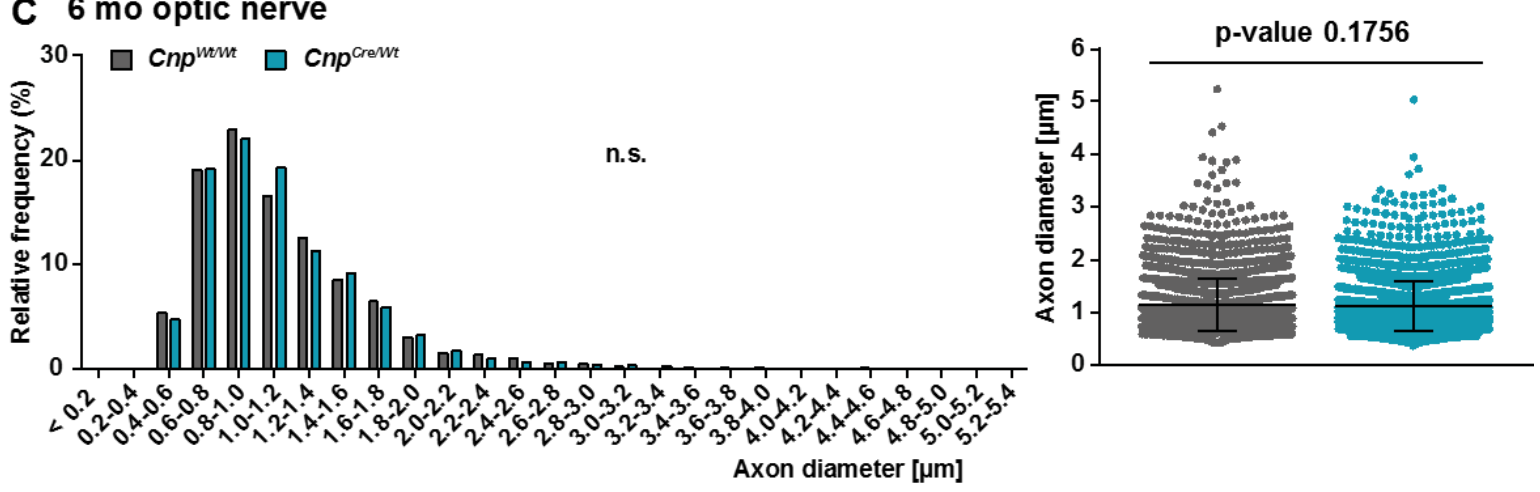

Figure 23: Axon diameter frequency distribution in SEPT8 deficient and Cnp ${ }^{C r e / W t}$ mice. (A) Frequency distribution analysis of optic nerve myelinated axons of electron micrographs at 6 mo of age indicate a slight trend towards larger axon diameter in Sept8nul//null mice. $n=5$ animals per genotype. Not significant according to Kolmogorov Smirnov test $(p=0.06254)$. (B) Frequency distribution analysis of optic nerve myelinated axons of

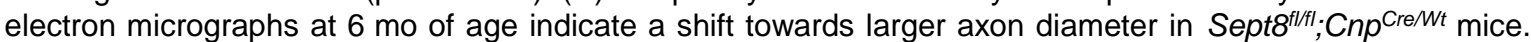
$\mathrm{n}=5$ animals per genotype. Significant according to Kolmogorov Smirnov test $(\mathrm{p}=<0.0001)$. (C) Frequency distribution analysis of optic nerve myelinated axons of electron micrographs at 6 mo of age indicate no shift towards larger axon diameter in $C n p^{C r e W t}$ mice. $\mathrm{n}=5$ animals per genotype. Not significant according to Kolmogorov Smirnov test $(p=0.1756)$. 


\section{Discussion}

\subsection{Is ANLN influencing septin filament assembly?}

In previous studies, septin filaments and anillin were shown to be associated with each other. For example, studies in yeast have shown that ANLN and septins co-localize within the cleavage furrow and thereby, enable proper cell division (Maddox et al., 2007, Oegema et al., 2000, Renshaw et al., 2014). Furthermore, in vitro studies have shown that ANLN acts as an adaptor to recruit septins onto membranes and that ANLN enhances PIP $_{2}$ mediated septin filament formation (Kinoshita et al., 2002, Liu et al., 2012), suggesting that septin filament formation relies on anillin. In vivo, it was shown that anillin also relies on septins, as the loss of septin filaments in CNS myelin led to a reduction of ANLN on protein level (Patzig et al., 2016). The reduction of ANLN was also seen in other tested mutants, which show reduced levels of myelin septins (Patzig et al., 2016). These results indicate that ANLN not only promotes septin filament formation, but also interacts with the filament to stabilize adaxonal myelin. To test whether ANLN serves a crucial function in myelin septin filament assembly in vivo, beyond its mere association with myelin septins, a novel line of mouse mutants (Anllilox/flox;Cnp Cre/Wt mice) was generated and analyzed. The presented results illustrate that the loss of ANLN from mature oligodendrocytes leads to a strong reduction of septins on protein level (Figure 12C and 13B). Moreover, as the mRNA abundance of septins is unaltered in Anln cKO mice (Figure 14), it is unlikely that the reduction is due to transcriptional regulation. It was shown that ANLN has a stabilizing function during cytogenesis (Liu et al., 2012), suggesting that ANLN could also stabilize the septin filament, thereby probably preventing its degradation. Another possibility is that ANLN facilitates septin filament formation, as it was shown that anillin enhances PIP $_{2}$ mediated septin filament formation in vitro (Kinoshita et al., 2002, Liu et al., 2012). Septin monomers and ANLN likely get degraded, if they are not incorporated into a filament (Patzig et al., 2016). The presented results suggest that the reduced septin abundance is caused by degradation of septin monomers. This could be either due to a loss of stability of the filament, or due to a hindered filament assembly.

To further test the influence of ANLN on septin filaments, it would be interesting to immunohistochemically visualize septin filaments in Anln cKO mice. By this, it could be analyzed if the septin filaments are altered in abundance or length due to the lack of ANLN. Based on the assumption that ANLN stabilizes the septin filament or facilitates septin filament assembly, it is likely that the abundance and length of the septin filaments are altered. The effect of ANLN on septin filament assembly could also be investigated by generating an ANLN overexpressing mouse line. It would be also interesting to analyze if ANLN would further enhance/stabilize the septin filament assembly, possibly leading to more abundant and longer filaments, which might further stabilize the myelin. 
It was reported that the loss of CNS myelin septin filaments lead to myelin outfoldings (Patzig et al., 2016). This could be confirmed in the presented study, as it is illustrated in Figure 15. As septins are also less abundant upon aging (Patzig et al., 2016), it would be interesting to test in ANLN overexpressing mice if the overexpression of ANLN could rescue the loss of the ANLN/septin filament in old mice. As the loss of the ANLN/septin filament coincides with myelin outfoldings and reduced nerve conduction velocity (Patzig et al., 2016) (Figure 17A), a rescue of the ANLN/septin filament might prevent these pathological phenotypes in aging.

\subsection{ANLN/septin filaments in development - who comes first?}

In several studies, it was shown that ANLN interacts with septins (Patzig et al., 2016, Liu et al., 2012, Renshaw et al., 2014, Kinoshita, 2003), but so far there is less known about the (developmental) expression chronology of these proteins. In Candida albicans, it was shown that the anillin-related Int1 and the Sep7 septin interact with each other during cytokinesis (Orellana-Munoz et al., 2018). Interestingly, the authors have presented, that the septin ring assembly occurred before the incorporation of Int1, suggesting that possibly the septin filament is build first and then stabilized by ANLN. In the presented data, it could be shown in immunoblots of P15, P18, P21, and P24 purified myelin that the abundance of ANLN and myelin septins have a similar chronology (Figure 10B). These results are in accordance with the immunoblot analysis in Patzig et al. (2016), where similar increasing abundances of septins were detected. Furthermore, in the results regarding the immunohistochemical analysis of P15, P21, and P28 optic nerves, it was shown that ANLN and SEPT8 are similar distributed and show a similar protein expression chronology (Figure 10A). It was seen that each filament-like labeling was positive for ANLN and SEPT8, and that the number of labeled filament-like structures increased from P21 to P28. Interestingly, filament-like labeling was detectable at P21, whereas in Patzig et al. (2016) no labeling was detectable at this time point. This result suggests, that the protein expression of septins is probably adjusted to the local needs of the axon and that there is a longer time frame of ANLN/septin arising. Unfortunately, the results did not shed light on the question whether ANLN or septins evolve first. To further analyze this question, it would be necessary to look at smaller time intervals like P19, P20, P21, and P22 in optic nerve longitudinal sections. Also, the immunoblot analysis of purified myelin could be adjusted, and earlier time points could be analyzed. Another possibility would be the usage of microfluidic chambers to model CNS myelination (Vaquie et al., 2018). In this system, the protein expression chronology of ANLN and septins could be analyzed in more detail using live-cell imaging techniques. In this experiment, it has to be considered that in oligodendroglial cultures, it was not possible to detect the formation of filament-like 
structures of septins (unpublished data). This could be due to too short incubation time, but also due to using an in vitro system, as it was detected that the adaxonal myelin layer, to which septins localize, is not modeled in oligodendroglial cultures. Furthermore, it was shown that septin filament formation arises in late stages of myelination. This mature state of an oligodendrocyte might not be reached in an in vitro system.

\subsection{Do myelin outfoldings decrease nerve conduction velocity?}

Myelin is known to increase nerve conduction velocity by blocking internodal current leakage and decreasing the transverse capacitance between the inside and outside of a nerve fiber (Hartline and Colman, 2007). The increased membrane resistance enables an increased velocity of signal propagation. With the so-called saltatory signal propagation, the $\mathrm{NCV}$ is increased from $10 \mathrm{~m} / \mathrm{sec}$ up to $150 \mathrm{~m} / \mathrm{sec}$. It is proposed that electrical sealing through two opposed membranes is playing a crucial role in decreasing the transverse capacitance and proportionately speeds impulse propagation along it. It is suggested that the sealing can be achieved by narrowing the conductive space between adjacent axonal and glial membranes, which means the cytoplasmic or extracytoplasmic space (Hartline and Colman, 2007). As depicted in Figure 17A, Anln cKO mice exhibit a decelerated nerve conduction velocity of about $15 \%$ in spinal cord compared to controls. Moreover, these mutants display specifically myelin outfoldings due to the lack of the ANLN/septin filament. 3D reconstruction of Anln cKO optic nerves illustrated that several axons exhibit myelin outfoldings (Figure 16C'). Another study estimates that if $6.5 \%$ of myelinated axons display myelin outfoldings of an average $10 \mu \mathrm{m}$ length, at least half of the myelinated segments probably have myelin outfoldings (Snaidero et al., 2014). Other studies showed similar results of reduced NCV in other mouse mutants, which exhibit myelin outfoldings and lack the ANLN/septin filament. For example, Sept8 $8^{f / f l} ; C n p^{C r e / W t}$ mice showed a decelerated nerve conduction velocity of about $20 \%$ in the spinal cord and depicted specifically myelin outfoldings (Patzig et al., 2016). Further, NCV measurements in mice showing more complex pathology (Cnp $p^{\text {null/null }}, M^{\text {null/null }}$, and $\left.P / p^{\text {null/null}}\right)$, including reduced ANLN/septin filament abundance and therefore also myelin outfoldings, indicated decelerated NCV in the spinal cord (unpublished data). In Patzig et al. (2016), it was suggested that myelin outfoldings impede the current flow along myelinated fibers and thereby, leading to the identified deceleration, keeping in mind that other unidentified secondary effects could also contribute to the deceleration. Moreover, it was shown that in optic nerves of PLP mutants the NCV is also reduced (Gutierrez et al., 1995). There it was suggested that the loosely packed myelin sheaths of PLP mutants lead to a functional deficit in conduction velocities of central fibers. The authors have shown that the measured NCV in developmental nerves is also reduced. This leads to the assumption that only complete myelination gives the basis 
for normal NCV (Gutierrez et al., 1995). Keeping in mind that also other factors could have an influence on NCV (Wu et al., 2012, Arancibia-Carcamo et al., 2017), the nodal structure and density was analyzed in the presented study (Figure 17B-G). The results display no alterations in the nodal structure, as well as in the node density, which is associated with the internode length (Ford et al., 2015). Thus, according to the presented data, decelerated NCV may be caused by myelin outfoldings, as the nodal and internodal structure is normal. Hypothetically, myelin outfoldings could provoke the lack of proper insulation and reduced membrane resistance along the axon, due to more cytoplasm along the space where the myelin folds out. This would lead to a partially lower membrane resistance where an increased transmembrane current could take place. That would result in a regional cation leak out, so that less cations would be available to depolarize other parts of the membrane. This would mean a slowdown of signal propagation. Considering the estimation that half of the myelinated segments display myelin outfoldings, the presented results strengthen the possibility of myelin outfoldings impeding signal propagation, even though other unidentified secondary effects could possibly contribute to the decelerated NCV. To directly test whether outfoldings reduce NCV, it would be necessary to patch an axon with an outfolding and record NCV. It is possible to measure NCV ex vivo in optic nerves, but it would be necessary to identify a single axon with a myelin outfolding and directly measure its NCV. Although it is possible to patch single axons, the identification of a myelin outfolding is not feasible, as in light microscopic applications, it was not possible to identify myelin outfoldings by labeling myelin. Up until now, the available techniques are not feasible to record NCV from single axons with previously identified myelin outfoldings.

\subsection{Does loss of ANLN lead to altered PIP2 levels in CNS myelin?}

Several studies report that ANLN, $\mathrm{PIP}_{2}$, and septins are associated with each other (Liu et al., 2012, Zhang et al., 1999, Bertin et al., 2010, Joberty et al., 2001, Patzig et al., 2016). It was shown that $\mathrm{PIP}_{2}$ head groups bind ANLN to recruit it to the cleavage furrow in vitro (Liu et al., 2012). Another in vitro study reported that $\mathrm{PIP}_{2}$ recruits mammalian SEPT4 onto the plasma membrane (Zhang et al., 1999). Furthermore, it was shown that $\mathrm{PIP}_{2}$ mediates submembranous septin filament polymerization in yeast (Bertin et al., 2010). In an in vivo model, it was shown that lack of PTEN in oligodendrocytes results in reduced $\mathrm{PIP}_{2}$ levels. This leads to reduced protein abundance of septins and ANLN, including increased myelin outfoldings besides other pathologies (Patzig et al., 2016, Goebbels et al., 2010). So far, $\mathrm{PIP}_{2}$ seems to affect myelin septin assembly via ANLN. The results illustrated in Figure 21 show reduced $\mathrm{PIP}_{2}$ levels in purified myelin of AnIn cKO mice compared to controls. This leads to the assumption that ANLN also has a role in $\mathrm{PIP}_{2}$ stabilization, possibly by binding to the head groups and thereby hindering modifications of $\mathrm{PIP}_{2}$. A possible modification is 
the conversion of $\mathrm{PIP}_{2}$ to $\mathrm{PIP}_{3}$ via PI3K. Thus, it would be interesting to analyze $\mathrm{PIP}_{3}$ levels in Anln cKO mice. As high levels of $\mathrm{PIP}_{3}$ induce active net growth of myelin sheaths via the Akt/mTOR pathway, it would be of further interest to analyze the pathway in more detail. One possibility would be an immunoblot analysis of purified myelin, testing the phosphorylation state of Akt and Erk, which are downstream effectors of PI3K.

\subsection{Myelin stability; a novel effector of axon diameter restriction?}

It is known that the axon diameter is influenced by several factors, e.g. radial growth of axons is promoted by myelination due to modulation of the phosphorylation of neurofilaments (NF) at internodes (Hsieh et al., 1994). The modulation leads to larger interfilament spacing, which coincides with an increased number of neurofilaments, resulting in larger axon diameters. The phosphorylation of NF-medium and NF-heavy, was also shown to play an important role in axonal diameter regulation (Garcia et al., 2003). Furthermore, it was shown that $M B P^{n u l l / n u l l}$ (shiverer) mice, in which oligodendrocytes only wrap loose layers around axons, resulting in little or no compact myelin (Readhead et al., 1987), exhibit smaller axon diameters. This reduction in axon diameters is caused by changes in microtubule stability and density (Kirkpatrick et al., 2001). In the illustrated results, it was shown that $A n I n$ cKO and $S e p t 8^{f / f t} ; C n p^{C r e / W t}$ mice display increased axon diameter at 6 mo of age (Figure 22B and 23B). Interestingly, this phenotype was only present in combination with CNP heterozygosity. CNP was reported to prevent premature closure of cytoplasmic channels in CNS myelin (Snaidero et al., 2014). These channels could provide transport routes, for example, for metabolites into the adaxonal myelin layer (Nave and Werner, 2014). The result leads to the suggestion that impairment of the ANLN/septin filament in CNS myelin together with premature closure of cytoplasmic channels leads to altered axon diameters. So far, the mechanism behind the increase is unclear. One speculative suggestion would be that oligodendrocytes form compact myelin, which is stabilized by the ANLN/septin filament, around axons and thereby give a spatial limitation due to the stable and robust ensheathment. To test if the CNP heterozygosity plays a role in this hypothesis, it would be needed to analyze Sept8 ${ }^{\text {null/null }} ; C_{n p} p^{C r e / W t}$ mice, as Sept $8^{\text {null/null }}$ mice did not display enlarged axon diameters (Figure 23A and C). This indicates that the loss of the ANLN/septin filament and consequently also the loss of myelin stability alone does not lead to increased diameter. Further taking into account that mice lacking compact myelin (shiverer) show a decrease in axon diameter, although they face no spatial limitation through compact myelin, indicates that the regulation is much more complex. This coincides with the finding that the maturation of the axonal cytoskeleton is not functional in shiverer mice as compact myelin plays a crucial role in this maturation (Brady et al., 1999). Additionally, it was shown that phosphatidylinositol phosphates, including $\mathrm{PIP}_{2}$, interact with 
neurofilaments (Kim et al., 2011), which are known to regulate axon diameter (Garcia et al., 2003). This suggests that the identified altered PIP 2 levels in Anln cKO mice (Figure 21) could also contribute to the observed increase in axonal diameter. To further analyze this,

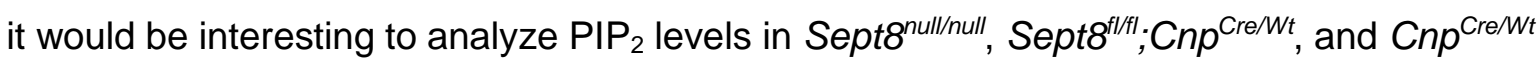
mice, to see if there is a correlation between enlarged axon diameters and altered $\mathrm{PIP}_{2}$ levels. Another important analysis would include an assessment of neurofilaments in mutants, which display altered axon diameter, as neurofilaments are involved in axon diameter regulation. Taken together, the AnIn cKO mouse might not be the perfect model to study axon diameter regulations, but may provide a new possible functionality of oligodendrocyte compact myelin for axons. To see whether stability provided by the ANLN/septin filament contributes to the axon growth via a spatial limitation, it would be interesting to analyze axon diameter in mice which express ANLN and septins earlier in development, to see if an earlier gained stability would restrict axonal growth to a certain extend. 


\section{References}

ALBERTS, B. 2002. Molecular Biology of the Cell, 4th edition [Online]. New York: Garland Science.

ARANCIBIA-CARCAMO, I. L., FORD, M. C., COSSELL, L., ISHIDA, K., TOHYAMA, K. \& ATTWELL, D. 2017. Node of Ranvier length as a potential regulator of myelinated axon conduction speed. Elife, 6.

ARROYO, E. J. \& SCHERER, S. S. 2000. On the molecular architecture of myelinated fibers. Histochem Cell Biol, 113, 1-18.

BAI, Y., ZHANG, X., KATONA, I., SAPORTA, M. A., SHY, M. E., O'MALLEY, H. A., ISOM, L. L., SUTER, U. \& LI, J. 2010. Conduction block in PMP22 deficiency. J Neurosci, 30, 600-8.

BAUMANN, N. \& PHAM-DINH, D. 2001. Biology of oligodendrocyte and myelin in the mammalian central nervous system. Physiol Rev, 81, 871-927.

BENDER, L., LO, H. S., LEE, H., KOKOJAN, V., PETERSON, V. \& BENDER, A. 1996. Associations among $\mathrm{PH}$ and $\mathrm{SH} 3$ domain-containing proteins and Rho-type GTPases in Yeast. J Cell Biol, 133, 879-94.

BENNETT, V. 2013. Functional organization of vertebrate plasma membrane, Current topics in membranes.

BERGHS, S., AGGUJARO, D., DIRKX, R., JR., MAKSIMOVA, E., STABACH, P., HERMEL, J. M., ZHANG, J. P., PHILBRICK, W., SLEPNEV, V., ORT, T. \& SOLIMENA, M. 2000. betalV spectrin, a new spectrin localized at axon initial segments and nodes of ranvier in the central and peripheral nervous system. J Cell Biol, 151, 985-1002.

BERTIN, A., MCMURRAY, M. A., THAI, L., GARCIA, G., 3RD, VOTIN, V., GROB, P., ALLYN, T., THORNER, J. \& NOGALES, E. 2010. Phosphatidylinositol4,5-bisphosphate promotes budding yeast septin filament assembly and organization. J Mol Biol, 404, 711-31.

BLACK, J. A. \& WAXMAN, S. G. 1988. The perinodal astrocyte. Glia, 1, 169-83.

BOYLE, M. E., BERGLUND, E. O., MURAI, K. K., WEBER, L., PELES, E. \& RANSCHT, B. 2001. Contactin orchestrates assembly of the septate-like junctions at the paranode in myelinated peripheral nerve. Neuron, 30, 38597.

BRADY, S. T., WITT, A. S., KIRKPATRICK, L. L., DE WAEGH, S. M., READHEAD, C., TU, P. H. \& LEE, V. M. 1999. Formation of compact myelin is required for maturation of the axonal cytoskeleton. J Neurosci, 19, 7278-88.

BRINKMANN, B. G., AGARWAL, A., SEREDA, M. W., GARRATT, A. N., MULLER, T., WENDE, H., STASSART, R. M., NAWAZ, S., HUMML, C., VELANAC, V., RADYUSHKIN, K., GOEBBELS, S., FISCHER, T. M., FRANKLIN, R. J., LAI, C., EHRENREICH, H., BIRCHMEIER, C., SCHWAB, M. H. \& NAVE, K. A. 
2008. Neuregulin-1/ErbB signaling serves distinct functions in myelination of the peripheral and central nervous system. Neuron, 59, 581-95.

CANTLEY, L. C. \& NEEL, B. G. 1999. New insights into tumor suppression: PTEN suppresses tumor formation by restraining the phosphoinositide 3kinase/AKT pathway. Proc Natl Acad Sci U S A, 96, 4240-5.

CAO, L., DING, X., YU, W., YANG, X., SHEN, S. \& YU, L. 2007. Phylogenetic and evolutionary analysis of the septin protein family in metazoan. FEBS Lett, $581,5526-32$.

CARDONA, A., SAALFELD, S., SCHINDELIN, J., ARGANDA-CARRERAS, I., PREIBISCH, S., LONGAIR, M., TOMANCAK, P., HARTENSTEIN, V. \& DOUGLAS, R. J. 2012. TrakEM2 software for neural circuit reconstruction. PLoS One, 7, e38011.

CHOMIAK, T. \& HU, B. 2009. What is the optimal value of the g-ratio for myelinated fibers in the rat CNS? A theoretical approach. PLoS One, 4, e7754.

CHOU, C. C. \& BUEHLER, M. J. 2012. Structure and mechanical properties of human trichocyte keratin intermediate filament protein. Biomacromolecules, $13,3522-32$.

COOPER, G. 2000. Structure and Organization of Actin Filaments, Sunderland (MA): Sinauer Associates.

DE WAEGH, S. M., LEE, V. M. \& BRADY, S. T. 1992. Local modulation of neurofilament phosphorylation, axonal caliber, and slow axonal transport by myelinating Schwann cells. Cell, 68, 451-63.

DECHAT, T., ADAM, S. A., TAIMEN, P., SHIMI, T. \& GOLDMAN, R. D. 2010. Nuclear lamins. Cold Spring Harb Perspect Biol, 2, a000547.

DEERINCK, T. J., BUSHONG, E. A., THOR, A. \& ELLISMAN, M. H. 2010. NCMIR METHODS FOR 3D EM: A NEW PROTOCOL FOR PREPARATION OF BIOLOGICAL SPECIMENS FOR SERIAL BLOCKFACE SCANNING ELECTRON MICROSCOPY - SBEM Protocol v7_01_10'. NCMIR. Available at: https://www.ncmir.ucsd.edu/sbem-protocol.

DIBAJ, P., ZSCHUNTZSCH, J., STEFFENS, H., SCHEFFEL, J., GORICKE, B., WEISHAUPT, J. H., LE MEUR, K., KIRCHHOFF, F., HANISCH, U. K., SCHOMBURG, E. D. \& NEUSCH, C. 2012. Influence of methylene blue on microglia-induced inflammation and motor neuron degeneration in the SOD1(G93A) model for ALS. PLoS One, 7, e43963.

EINHEBER, S., ZANAZZI, G., CHING, W., SCHERER, S., MILNER, T. A., PELES, E. \& SALZER, J. L. 1997. The axonal membrane protein Caspr, a homologue of neurexin IV, is a component of the septate-like paranodal junctions that assemble during myelination. J Cell Biol, 139, 1495-506.

FIELD, C. M. \& ALBERTS, B. M. 1995. Anillin, a contractile ring protein that cycles from the nucleus to the cell cortex. J Cell Biol, 131, 165-78. 
FIELDS, R. D. \& STEVENS-GRAHAM, B. 2002. New insights into neuron-glia communication. Science, 298, 556-62.

FORD, M. C., ALEXANDROVA, O., COSSELL, L., STANGE-MARTEN, A., SINCLAIR, J., KOPP-SCHEINPFLUG, C., PECKA, M., ATTWELL, D. \& GROTHE, B. 2015. Tuning of Ranvier node and internode properties in myelinated axons to adjust action potential timing. Nat Commun, 6, 8073.

FUCHTBAUER, A., LASSEN, L. B., JENSEN, A. B., HOWARD, J., QUIROGA ADE, S., WARMING, S., SORENSEN, A. B., PEDERSEN, F. S. \& FUCHTBAUER, E. M. 2011. Septin9 is involved in septin filament formation and cellular stability. Biol Chem, 392, 769-77.

FUNFSCHILLING, U., SUPPLIE, L. M., MAHAD, D., BORETIUS, S., SAAB, A. S., EDGAR, J., BRINKMANN, B. G., KASSMANN, C. M., TZVETANOVA, I. D., MOBIUS, W., DIAZ, F., MEIJER, D., SUTER, U., HAMPRECHT, B., SEREDA, M. W., MORAES, C. T., FRAHM, J., GOEBBELS, S. \& NAVE, K. A. 2012. Glycolytic oligodendrocytes maintain myelin and long-term axonal integrity. Nature, 485, 517-21.

GALLYAS, F. 1979. Silver staining of myelin by means of physical development. Neurol Res, 1, 203-9.

GARCIA, M. L., LOBSIGER, C. S., SHAH, S. B., DEERINCK, T. J., CRUM, J., YOUNG, D., WARD, C. M., CRAWFORD, T. O., GOTOW, T., UCHIYAMA, Y., ELLISMAN, M. H., CALCUTT, N. A. \& CLEVELAND, D. W. 2003. NF-M is an essential target for the myelin-directed "outside-in" signaling cascade that mediates radial axonal growth. J Cell Biol, 163, 1011-20.

GILDEN, J. \& KRUMMEL, M. F. 2010. Control of cortical rigidity by the cytoskeleton: emerging roles for septins. Cytoskeleton (Hoboken), 67, 477-86.

GOEBBELS, S., OLTROGGE, J. H., KEMPER, R., HEILMANN, I., BORMUTH, I., WOLFER, S., WICHERT, S. P., MOBIUS, W., LIU, X., LAPPE-SIEFKE, C., ROSSNER, M. J., GROSZER, M., SUTER, U., FRAHM, J., BORETIUS, S. \& NAVE, K. A. 2010. Elevated phosphatidylinositol 3,4,5-trisphosphate in glia triggers cell-autonomous membrane wrapping and myelination. $J$ Neurosci, 30, 8953-64.

GOEBBElS, S., WIESER, G. L., PIEPER, A., SPITZER, S., WEEGE, B., YAN, K., EDGAR, J. M., YAGENSKY, O., WICHERT, S. P., AGARWAL, A., KARRAM, K., RENIER, N., TESSIER-LAVIGNE, M., ROSSNER, M. J., KARADOTTIR, R. T. \& NAVE, K. A. 2017. A neuronal PI(3,4,5)P3-dependent program of oligodendrocyte precursor recruitment and myelination. Nat Neurosci, 20, 1015.

GOLLAN, L., SABANAY, H., POLIAK, S., BERGLUND, E. O., RANSCHT, B. \& PELES, E. 2002. Retention of a cell adhesion complex at the paranodal junction requires the cytoplasmic region of Caspr. $J$ Cell Biol, 157, 1247-56.

GRIFFITHS, I., KLUGMANN, M., ANDERSON, T., YOOL, D., THOMSON, C., SCHWAB, M. H., SCHNEIDER, A., ZIMMERMANN, F., MCCULLOCH, M., 
NADON, N. \& NAVE, K. A. 1998. Axonal swellings and degeneration in mice lacking the major proteolipid of myelin. Science, 280, 1610-3.

GUTIERREZ, R., BOISON, D., HEINEMANN, U. \& STOFFEL, W. 1995. Decompaction of Cns Myelin Leads to a Reduction of the ConductionVelocity of Action-Potentials in Optic-Nerve. Neuroscience Letters, 195, 9396.

HALL, P. A. \& RUSSELL, S. E. 2004. The pathobiology of the septin gene family. $J$ Pathol, 204, 489-505.

HALL, P. A. \& RUSSELL, S. E. 2012. Mammalian septins: dynamic heteromers with roles in cellular morphogenesis and compartmentalization. $J$ Pathol, 226, 287-99.

HANISCH, U. K. 2002. Microglia as a source and target of cytokines. Glia, 40, 14055.

HANISCH, U. K. \& KETTENMANN, H. 2007. Microglia: active sensor and versatile effector cells in the normal and pathologic brain. Nat Neurosci, 10, 1387-94.

HARTLINE, D. K. \& COLMAN, D. R. 2007. Rapid conduction and the evolution of giant axons and myelinated fibers. Curr Biol, 17, R29-35.

HERRMANN, H., BAR, H., KREPLAK, L., STRELKOV, S. V. \& AEBI, U. 2007. Intermediate filaments: from cell architecture to nanomechanics. Nat Rev Mol Cell Biol, 8, 562-73.

HILDEBRAND, C. \& HAHN, R. 1978. Relation between myelin sheath thickness and axon size in spinal cord white matter of some vertebrate species. J Neurol Sci, 38, 421-34.

HIROKAWA, N. \& TAKEMURA, R. 2005. Molecular motors and mechanisms of directional transport in neurons. Nat Rev Neurosci, 6, 201-14.

HSIEH, S. T., KIDD, G. J., CRAWFORD, T. O., XU, Z., LIN, W. M., TRAPP, B. D., CLEVELAND, D. W. \& GRIFFIN, J. W. 1994. Regional modulation of neurofilament organization by myelination in normal axons. $J$ Neurosci, 14, $6392-401$.

JAEGLE, M., GHAZVINI, M., MANDEMAKERS, W., PIIRSOO, M., DRIEGEN, S., LEVAVASSEUR, F., RAGHOENATH, S., GROSVELD, F. \& MEIJER, D. 2003. The POU proteins Brn-2 and Oct-6 share important functions in Schwann cell development. Genes Dev, 17, 1380-91.

JOBERTY, G., PERLUNGHER, R. R., SHEFFIELD, P. J., KINOSHITA, M., NODA, M., HAYSTEAD, T. \& MACARA, I. G. 2001. Borg proteins control septin organization and are negatively regulated by Cdc42. Nat Cell Biol, 3, 861-6.

JUNG, M., SOMMER, I., SCHACHNER, M. \& NAVE, K. A. 1996. Monoclonal antibody $\mathrm{O} 10$ defines a conformationally sensitive cell-surface epitope of proteolipid protein (PLP): evidence that PLP misfolding underlies dysmyelination in mutant mice. J Neurosci, 16, 7920-9. 
KETTENMANN, H., HANISCH, U. K., NODA, M. \& VERKHRATSKY, A. 2011. Physiology of microglia. Physiol Rev, 91, 461-553.

KETTENMANN, H. \& RANSOM, B. R. 2005. Neuroglia, Oxford University Press.

KIM, S. K., KIM, H., YANG, Y. R., SUH, P. G. \& CHANG, J. S. 2011. Phosphatidylinositol phosphates directly bind to neurofilament light chain (NF-L) for the regulation of NF-L self assembly. Exp Mol Med, 43, 153-60.

KING, R. 2013. Microscopic anatomy: normal structure. Handb Clin Neurol, 115, 727.

KINOSHITA, M. 2003. Assembly of Mammalian Septins. Journal of Biochemistry, 134, 491-496.

KINOSHITA, M., FIELD, C. M., COUGHLIN, M. L., STRAIGHT, A. F. \& MITCHISON, T. J. 2002. Self- and actin-templated assembly of Mammalian septins. Dev Cell, 3, 791-802.

KIRCHHOFF, F., DRINGEN, R. \& GIAUME, C. 2001. Pathways of neuron-astrocyte interactions and their possible role in neuroprotection. Eur Arch Psychiatry Clin Neurosci, 251, 159-69.

KIRKPATRICK, L. L., WITT, A. S., PAYNE, H. R., SHINE, H. D. \& BRADY, S. T. 2001. Changes in microtubule stability and density in myelin-deficient shiverer mouse CNS axons. J Neurosci, 21, 2288-97.

KISSEL, H., GEORGESCU, M. M., LARISCH, S., MANOVA, K., HUNNICUTT, G. R. \& STELLER, H. 2005. The Sept4 septin locus is required for sperm terminal differentiation in mice. Dev Cell, 8, 353-64.

KLUGMANN, M., SCHWAB, M. H., PUHLHOFER, A., SCHNEIDER, A., ZIMMERMANN, F., GRIFFITHS, I. R. \& NAVE, K. A. 1997. Assembly of CNS myelin in the absence of proteolipid protein. Neuron, 18, 59-70.

KONIG, S., HOFFMANN, M., MOSBLECH, A. \& HEILMANN, I. 2008. Determination of content and fatty acid composition of unlabeled phosphoinositide species by thin-layer chromatography and gas chromatography. Anal Biochem, 378, 197-201.

KREMER, J. R., MASTRONARDE, D. N. \& MCINTOSH, J. R. 1996. Computer visualization of three-dimensional image data using IMOD. J Struct Biol, 116, 71-6.

KUHLENBAUMER, G., HANNIBAL, M. C., NELIS, E., SCHIRMACHER, A., VERPOORTEN, N., MEULEMAN, J., WATTS, G. D., DE VRIENDT, E., YOUNG, P., STOGBAUER, F., HALFTER, H., IROBI, J., GOOSSENS, D., DEL-FAVERO, J., BETZ, B. G., HOR, H., KURLEMANN, G., BIRD, T. D., AIRAKSINEN, E., MONONEN, T., SERRADELL, A. P., PRATS, J. M., VAN BROECKHOVEN, C., DE JONGHE, P., TIMMERMAN, V., RINGELSTEIN, E. B. \& CHANCE, P. F. 2005. Mutations in SEPT9 cause hereditary neuralgic amyotrophy. Nat Genet, 37, 1044-6. 
KWITNY, S., KLAUS, A. V. \& HUNNICUTT, G. R. 2010. The annulus of the mouse sperm tail is required to establish a membrane diffusion barrier that is engaged during the late steps of spermiogenesis. Biol Reprod, 82, 669-78.

LAPPE-SIEFKE, C., GOEBBELS, S., GRAVEL, M., NICKSCH, E., LEE, J., BRAUN, P. E., GRIFFITHS, I. R. \& NAVE, K. A. 2003. Disruption of Cnp1 uncouples oligodendroglial functions in axonal support and myelination. Nat Genet, 33, 366-74.

LAROCCA, J. N. \& NORTON, W. T. 2007. Isolation of myelin. Curr Protoc Cell Biol, Chapter 3, Unit3 25.

LEE, J. C., SHIN, J. H., PARK, B. W., KIM, G. S., KIM, J. C., KANG, K. S. \& CHA, C. I. 2012. Region-specific changes in the immunoreactivity of SIRT1 expression in the central nervous system of SOD1(G93A) transgenic mice as an in vivo model of amyotrophic lateral sclerosis. Brain Res, 1433, 20-8.

LEE, J. Y., KIM, M. J., LI, L., VELUMIAN, A. A., AUI, P. M., FEHLINGS, M. G. \& PETRATOS, S. 2017. Nogo receptor 1 regulates Caspr distribution at axoglial units in the central nervous system. Sci Rep, 7, 8958.

LEITE, S. C., SAMPAIO, P., SOUSA, V. F., NOGUEIRA-RODRIGUES, J., PINTOCOSTA, R., PETERS, L. L., BRITES, P. \& SOUSA, M. M. 2016. The ActinBinding Protein alpha-Adducin Is Required for Maintaining Axon Diameter. Cell Rep, 15, 490-498.

LINNINGTON, C., WEBB, M. \& WOODHAMS, P. L. 1984. A novel myelinassociated glycoprotein defined by a mouse monoclonal antibody. $J$ Neuroimmunol, 6, 387-96.

LIU, J., FAIRN, G. D., CECCARELLI, D. F., SICHERI, F. \& WILDE, A. 2012. Cleavage Furrow Organization Requires PIP(2)-Mediated Recruitment of Anillin. Curr Biol, 22, 64-9.

LOW, C. \& MACARA, I. G. 2006. Structural analysis of septin 2, 6, and 7 complexes. J Biol Chem, 281, 30697-706.

MACARA, I. G., BALDARELLI, R., FIELD, C. M., GLOTZER, M., HAYASHI, Y., HSU, S. C., KENNEDY, M. B., KINOSHITA, M., LONGTINE, M., LOW, C., MALTAIS, L. J., MCKENZIE, L., MITCHISON, T. J., NISHIKAWA, T., NODA, M., PETTY, E. M., PEIFER, M., PRINGLE, J. R., ROBINSON, P. J., ROTH, D., RUSSELL, S. E., STUHLMANN, H., TANAKA, M., TANAKA, T., TRIMBLE, W. S., WARE, J., ZELEZNIK-LE, N. J. \& ZIEGER, B. 2002. Mammalian septins nomenclature. Mol Biol Cell, 13, 4111-3.

MADDOX, A. S., LEWELLYN, L., DESAI, A. \& OEGEMA, K. 2007. Anillin and the septins promote asymmetric ingression of the cytokinetic furrow. Dev Cell, $12,827-35$.

MENDOZA, M., HYMAN, A. A. \& GLOTZER, M. 2002. GTP binding induces filament assembly of a recombinant septin. Curr Biol, 12, 1858-63. 
MICHAILOV, G. V., SEREDA, M. W., BRINKMANN, B. G., FISCHER, T. M., HAUG, B., BIRCHMEIER, C., ROLE, L., LAI, C., SCHWAB, M. H. \& NAVE, K. A. 2004. Axonal neuregulin-1 regulates myelin sheath thickness. Science, 304, 700-3.

MIESCHER, G. C., LUTZELSCHWAB, R., ERNE, B., FERRACIN, F., HUBER, S. \& STECK, A. J. 1997. Reciprocal expression of myelin-associated glycoprotein splice variants in the adult human peripheral and central nervous systems. Brain Res Mol Brain Res, 52, 299-306.

MILLER, K. G., FIELD, C. M. \& ALBERTS, B. M. 1989. Actin-binding proteins from Drosophila embryos: a complex network of interacting proteins detected by F-actin affinity chromatography. J Cell Biol, 109, 2963-75.

MOSTOWY, S. \& COSSART, P. 2012. Septins: the fourth component of the cytoskeleton. Nat Rev Mol Cell Biol.

NAVE, K. A. 2010. Myelination and support of axonal integrity by glia. Nature, 468, 244-52.

NAVE, K. A. \& WERNER, H. B. 2014. Myelination of the nervous system: mechanisms and functions. Annu Rev Cell Dev Biol, 30, 503-33.

NIMMERJAHN, A., KIRCHHOFF, F. \& HELMCHEN, F. 2005. Resting microglial cells are highly dynamic surveillants of brain parenchyma in vivo. Science, 308, 1314-8.

NORTON, W. T. \& PODUSLO, S. E. 1973. Myelination in rat brain: method of myelin isolation. J Neurochem, 21, 749-57.

ODA, T., IWASA, M., AIHARA, T., MAEDA, Y. \& NARITA, A. 2009. The nature of the globular- to fibrous-actin transition. Nature, 457, 441-5.

OEGEMA, K., SAVOIAN, M. S., MITCHISON, T. J. \& FIELD, C. M. 2000. Functional analysis of a human homologue of the Drosophila actin binding protein anillin suggests a role in cytokinesis. J Cell Biol, 150, 539-52.

OGAWA, Y., OSES-PRIETO, J., KIM, M. Y., HORRESH, I., PELES, E., BURLINGAME, A. L., TRIMMER, J. S., MEIJER, D. \& RASBAND, M. N. 2010. ADAM22, a Kv1 channel-interacting protein, recruits membraneassociated guanylate kinases to juxtaparanodes of myelinated axons. $J$ Neurosci, 30, 1038-48.

$\mathrm{OH}$, Y. \& BI, E. 2011. Septin structure and function in yeast and beyond. Trends Cell Biol, 21, 141-8.

ORELLANA-MUNOZ, S., DUENAS-SANTERO, E., ARNAIZ-PITA, Y., DEL REY, F., CORREA-BORDES, J. \& VAZQUEZ DE ALDANA, C. R. 2018. The anillinrelated Int1 protein and the Sep7 septin collaborate to maintain cellular ploidy in Candida albicans. Sci Rep, 8, 2257.

PALANDRI, A., SALVADOR, V. R., WOJNACKI, J., VIVINETTO, A. L., SCHNAAR, R. L. \& LOPEZ, P. H. 2015. Myelin-associated glycoprotein modulates 
apoptosis of motoneurons during early postnatal development via $\mathrm{NgR/p75(NTR)}$ receptor-mediated activation of RhoA signaling pathways. Cell Death Dis, 6, e1876.

PATZIG, J., ERWIG, M. S., TENZER, S., KUSCH, K., DIBAJ, P., MOBIUS, W., GOEBBELS, S., SCHAEREN-WIEMERS, N., NAVE, K. A. \& WERNER, H. B. 2016. Septin/anillin filaments scaffold central nervous system myelin to accelerate nerve conduction. Elife, 5 .

PIEKNY, A. J. \& GLOTZER, M. 2008. Anillin is a scaffold protein that links RhoA, actin, and myosin during cytokinesis. Curr Biol, 18, 30-6.

PIEKNY, A. J. \& MADDOX, A. S. 2010. The myriad roles of Anillin during cytokinesis. Semin Cell Dev Biol, 21, 881-91.

POLIAK, S. \& PELES, E. 2003. The local differentiation of myelinated axons at nodes of Ranvier. Nat Rev Neurosci, 4, 968-80.

POLLARD, T. D. 2017. Tribute to Fumio Oosawa the pioneer in actin biophysics. Cytoskeleton (Hoboken), 74, 446-449.

RASBAND, M. N. 2011. Composition, assembly, and maintenance of excitable membrane domains in myelinated axons. Semin Cell Dev Biol, 22, 178-84.

READHEAD, C., POPKO, B., TAKAHASHI, N., SHINE, H. D., SAAVEDRA, R. A., SIDMAN, R. L. \& HOOD, L. 1987. Expression of a myelin basic protein gene in transgenic shiverer mice: correction of the dysmyelinating phenotype. Cell, $48,703-12$.

RENSHAW, M. J., LIU, J., LAVOIE, B. D. \& WILDE, A. 2014. Anillin-dependent organization of septin filaments promotes intercellular bridge elongation and Chmp4B targeting to the abscission site. Open Biol, 4, 130190.

RICHARDSON, K. C., JARETT, L. \& FINKE, E. H. 1960. Embedding in epoxy resins for ultrathin sectioning in electron microscopy. Stain Technol, 35, 313-23.

RIVEST, S. 2009. Regulation of innate immune responses in the brain. Nat Rev Immunol, 9, 429-39.

RUSHTON, W. A. 1951. A theory of the effects of fibre size in medullated nerve. $J$ Physiol, 115, 101-22.

SALZER, J. L. 2003. Polarized domains of myelinated axons. Neuron, 40, 297-318.

SALZER, J. L., BROPHY, P. J. \& PELES, E. 2008. Molecular domains of myelinated axons in the peripheral nervous system. Glia, 56, 1532-40.

SCHINDELIN, J., ARGANDA-CARRERAS, I., FRISE, E., KAYNIG, V., LONGAIR, M., PIETZSCH, T., PREIBISCH, S., RUEDEN, C., SAALFELD, S., SCHMID, B., TINEVEZ, J. Y., WHITE, D. J., HARTENSTEIN, V., ELICEIRI, K., TOMANCAK, P. \& CARDONA, A. 2012. Fiji: an open-source platform for biological-image analysis. Nat Methods, 9, 676-82. 
SEIDL, A. H. 2014. Regulation of conduction time along axons. Neuroscience, 276, 126-34.

SHARMA, K. Z., GUIXIN; LI, SHUXIN 2015. Astrogliosis and Axonal Regeneration, Academic Press.

SHERMAN, D. L., TAIT, S., MELROSE, S., JOHNSON, R., ZONTA, B., COURT, F. A., MACKLIN, W. B., MEEK, S., SMITH, A. J., COTTRELL, D. F. \& BROPHY, P. J. 2005. Neurofascins are required to establish axonal domains for saltatory conduction. Neuron, 48, 737-42.

SIRAJUDDIN, M., FARKASOVSKY, M., HAUER, F., KUHLMANN, D., MACARA, I. G., WEYAND, M., STARK, H. \& WITTINGHOFER, A. 2007. Structural insight into filament formation by mammalian septins. Nature, 449, 311-5.

SNAIDERO, N., MOBIUS, W., CZOPKA, T., HEKKING, L. H., MATHISEN, C., VERKLEIJ, D., GOEBBELS, S., EDGAR, J., MERKLER, D., LYONS, D. A., NAVE, K. A. \& SIMONS, M. 2014. Myelin Membrane Wrapping of CNS Axons by $\mathrm{PI}(3,4,5) \mathrm{P} 3-D e p e n d e n t$ Polarized Growth at the Inner Tongue. Cell, 156, 277-90.

STILES, B., GROSZER, M., WANG, S., JIAO, J. \& WU, H. 2004. PTENless means more. Dev Biol, 273, 175-84.

STRAIGHT, A. F., FIELD, C. M. \& MITCHISON, T. J. 2005. Anillin binds nonmuscle myosin II and regulates the contractile ring. Mol Biol Cell, 16, 193-201.

STREIT, W. J., CONDE, J. R., FENDRICK, S. E., FLANARY, B. E. \& MARIANI, C. L. 2005. Role of microglia in the central nervous system's immune response. Neurol Res, 27, 685-91.

SUZUKI, A., NAKANO, T., MAK, T. W. \& SASAKI, T. 2008. Portrait of PTEN: messages from mutant mice. Cancer Sci, 99, 209-13.

TADA, T., SIMONETTA, A., BATTERTON, M., KINOSHITA, M., EDBAUER, D. \& SHENG, M. 2007. Role of Septin cytoskeleton in spine morphogenesis and dendrite development in neurons. Curr Biol, 17, 1752-8.

TANAKA-TAKIGUCHI, Y., KINOSHITA, M. \& TAKIGUCHI, K. 2009. Septinmediated uniform bracing of phospholipid membranes. Curr Biol, 19, 140-5.

THURNHERR, T., BENNINGER, Y., WU, X., CHROSTEK, A., KRAUSE, S. M., NAVE, K. A., FRANKLIN, R. J., BRAKEBUSCH, C., SUTER, U. \& RELVAS, J. B. 2006. Cdc42 and Rac1 signaling are both required for and act synergistically in the correct formation of myelin sheaths in the CNS. $J$ Neurosci, 26, 10110-9.

TOOLEY, A. J., GILDEN, J., JACOBELLI, J., BEEMILLER, P., TRIMBLE, W. S., KINOSHITA, M. \& KRUMMEL, M. F. 2009. Amoeboid T lymphocytes require the septin cytoskeleton for cortical integrity and persistent motility. Nat Cell Biol, 11, 17-26. 
TOWBIN, H., STAEHELIN, T. \& GORDON, J. 1979. Electrophoretic transfer of proteins from polyacrylamide gels to nitrocellulose sheets: procedure and some applications. Proc Natl Acad Sci U S A, 76, 4350-4.

TOYA, M., IINO, Y. \& YAMAMOTO, M. 1999. Fission yeast Pob1p, which is homologous to budding yeast Boi proteins and exhibits subcellular localization close to actin patches, is essential for cell elongation and separation. Mol Biol Cell, 10, 2745-57.

TRAPP, B. D., ANDREWS, S. B., COOTAUCO, C. \& QUARLES, R. 1989. The myelin-associated glycoprotein is enriched in multivesicular bodies and periaxonal membranes of actively myelinating oligodendrocytes. J Cell Biol, 109, 2417-26.

TRAPP, B. D. \& QUARLES, R. H. 1982. Presence of the myelin-associated glycoprotein correlates with alterations in the periodicity of peripheral myelin. J Cell Biol, 92, 877-82.

TSANG, C. W., ESTEY, M. P., DICICCIO, J. E., XIE, H., PATTERSON, D. \& TRIMBLE, W. S. 2011. Characterization of presynaptic septin complexes in mammalian hippocampal neurons. Biol Chem, 392, 739-49.

VALE, R. D. 2003. The molecular motor toolbox for intracellular transport. Cell, 112, 467-80.

VAQUIE, A., SAUVAIN, A. \& JACOB, C. 2018. Modeling PNS and CNS Myelination Using Microfluidic Chambers. Methods Mol Biol, 1791, 157-168.

VERSELE, M. \& THORNER, J. 2005. Some assembly required: yeast septins provide the instruction manual. Trends Cell Biol, 15, 414-24.

WALKER, R. A., O'BRIEN, E. T., PRYER, N. K., SOBOEIRO, M. F., VOTER, W. A., ERICKSON, H. P. \& SALMON, E. D. 1988. Dynamic instability of individual microtubules analyzed by video light microscopy: rate constants and transition frequencies. J Cell Biol, 107, 1437-48.

WANG IP, C., KRONER, A., FISCHER, S., BERGHOFF, M., KOBSAR, I., MAURER, M. \& MARTINI, R. 2006. Role of immune cells in animal models for inherited peripheral neuropathies. Neuromolecular Med, 8, 175-90.

WANG, K. C., KIM, J. A., SIVASANKARAN, R., SEGAL, R. \& HE, Z. 2002. P75 interacts with the Nogo receptor as a co-receptor for Nogo, MAG and OMgp. Nature, 420, 74-8.

WU, L. M., WILLIAMS, A., DELANEY, A., SHERMAN, D. L. \& BROPHY, P. J. 2012. Increasing internodal distance in myelinated nerves accelerates nerve conduction to a flat maximum. Curr Biol, 22, 1957-61.

XIE, Y., VESSEY, J. P., KONECNA, A., DAHM, R., MACCHI, P. \& KIEBLER, M. A. 2007. The GTP-binding protein Septin 7 is critical for dendrite branching and dendritic-spine morphology. Curr Biol, 17, 1746-51. 
YIN, X., CRAWFORD, T. O., GRIFFIN, J. W., TU, P., LEE, V. M., LI, C., RODER, J. \& TRAPP, B. D. 1998. Myelin-associated glycoprotein is a myelin signal that modulates the caliber of myelinated axons. J Neurosci, 18, 1953-62.

YUAN, A., SASAKI, T., KUMAR, A., PETERHOFF, C. M., RAO, M. V., LIEM, R. K., JULIEN, J. P. \& NIXON, R. A. 2012. Peripherin is a subunit of peripheral nerve neurofilaments: implications for differential vulnerability of CNS and peripheral nervous system axons. J Neurosci, 32, 8501-8.

ZHANG, J., KONG, C., XIE, H., MCPHERSON, P. S., GRINSTEIN, S. \& TRIMBLE, W. S. 1999. Phosphatidylinositol polyphosphate binding to the mammalian septin H5 is modulated by GTP. Curr Biol, 9, 1458-67.

ZHANG, Y., CHEN, K., SLOAN, S. A., BENNETT, M. L., SCHOLZE, A. R., O'KEEFFE, S., PHATNANI, H. P., GUARNIERI, P., CANEDA, C., RUDERISCH, N., DENG, S., LIDDELOW, S. A., ZHANG, C., DANEMAN, R., MANIATIS, T., BARRES, B. A. \& WU, J. Q. 2014. An RNA-sequencing transcriptome and splicing database of glia, neurons, and vascular cells of the cerebral cortex. J Neurosci, 34, 11929-47.

ZHOU, D., LAMBERT, S., MALEN, P. L., CARPENTER, S., BOLAND, L. M. \& BENNETT, V. 1998. AnkyrinG is required for clustering of voltage-gated $\mathrm{Na}$ channels at axon initial segments and for normal action potential firing. $J$ Cell Biol, 143, 1295-304. 


\section{Addendum}

\subsection{Abstract}

Septins are a family of highly conserved filament-forming GTP-binding proteins. Some members of this protein family were identified to be abundant in myelin (e.g. SEPT2, SEPT7, SEPT8, SEPT9), in which they support long-term stability, at least in the CNS. In humans, mutations of the SEPT9 gene cause hereditary neuralgic amyotrophy (HNA), an autosomal dominant disorder, which predominantly affects the peripheral brachial plexus. Mice carrying a point mutation in the Sept9 gene, which is often seen in human HNA, and mice conditionally lacking SEPT9 or SEPT2 in Schwann cells were analyzed to possibly generate the first mouse model of HNA and to further investigate the biology of septins in vivo. Sequencing of the mutated gene region confirmed the point mutation in experimental animals. Immunoblots, and immunohistochemical labeling of teased fibers were used to analyze possible alterations in SEPT9 abundance and localization. By immunohistochemical labeling, septins were found to localize to paranodal segments adjacent to the nodes of Ranvier, the outer rim of Schmidt-Lanterman incisures, and the bands of Cajal. In all analyzed regions, no alterations of septin localization or abundance were detectable. Furthermore, Sept $9^{R 88 W / W t}$ mice showed unaltered numbers of myelinated axons, as well as unaltered myelin ultrastructure in sciatic nerves at P75. Mice lacking SEPT2 or SEPT9 from Schwann cells were analyzed on electron microscopic level at P4, $\mathrm{P} 14$, and 6 mo of age. No signs of impaired developmental myelination or pathology were identifiable. According to the analyses, Sept9 or Sept2 mutant mice, as well as mice carrying the point mutation showed no HNA-related abnormalities. This may reflect that a second impact, such as nerve constriction or inflammation might be necessary to trigger the onset of pathology. Taken together, the investigation of mice lacking SEPT2 or SEPT9 from Schwann cells or carrying a point mutation provide a valuable basis for further analysis aiming to understand the pathology of HNA.

\subsection{Introduction}

\subsubsection{Septins in the peripheral nervous system}

Mass spectrometry of purified myelin of sciatic nerves showed that in the peripheral nervous system (PNS), SEPT2, SEPT7, and SEPT11 are the most abundant septins. SEPT9 was found in a comparatively low abundance (Patzig et al., 2011). With immunohistochemical analysis of sciatic nerve teased fibers, SEPT2, SEPT7, SEPT8, SEPT9, and SEPT11 were detected. All of them localized to the paranodal myelin compartment (flanking the nodes of Ranvier) and also abaxonally in the outermost rim of Schmidt-Lanterman incisures and the bands of Cajal (Buser et al., 2009; Ogawa and Rasband, 2009; Patzig et al., 2011). Previous experiments showed that in PNS myelin SEPT2 is essential for the presence of other PNS 
myelin septins, as mice lacking SEPT2 from Schwann cells have strongly reduced protein abundances of all other PNS myelin septins (unpublished data).

\subsubsection{Hereditary neuralgic amyotrophy}

Hereditary neuralgic amyotrophy (HNA) is an autosomal dominant disorder characterized by recurrent episodes of focal neuropathy of the brachial plexus (Meuleman et al., 2001). The majority of HNA cases are caused by mutations affecting the SEPT9 gene on chromosome 17q25, thereby a point mutation of Arginine 88 to Tryptophan (R88W) is often seen (Kuhlenbäumer, et al., 2005; PMID 16186812; Meulemann et al., 2001; PMID 10602368). Owing to the lack of biopsy material and a rodent model, the pathobiology of HNA caused by SEPT9 mutations, has remained largely unknown at the molecular, cellular, and histological level.

The onset of the disease occurs usually in the $2^{\text {nd }}$ to $3^{\text {rd }}$ decade, whereby a single episode typically starts with pain in the affected arm. Weakness and sensory disturbances, which develop within days after onset of the pain, are also characteristic for this phenotype. Often, early in an episode, prominent atrophy of the affected muscles develops. The recover begins weeks to month after onset and can take months to years. There are different degrees of recovery, which vary from moderate to excellent. The next episode then can affect the same limb as well as the opposite one. Recurrent episodes in the same limb can lead to incomplete recovery. In HNA, any part of the brachial plexus can be affected, with a predilection for the upper trunk which enervates the proximal upper limb muscle. All in all, the classical course of the disease can be described as relapsing-remitting (Chance, 2006; van Alfen, 2011).

\subsection{Results}

\subsubsection{Analysis of Sept9R88W/Wt mice}

\subsubsection{Point mutation has no effect on septin protein abundances}

As SEPT9 mutations are often seen in HNA patients, especially the R88W point mutation, Sept ${ }^{R 88 W / W t}$ mice were analyzed at P75. When breeding heterozygous with heterozygous mice, it was noticed that no homozygous mice were born (Addendum Figure 1A) indicating that carrying the point mutation on both Sept9 alleles is embryonic lethal. Consequently, only Sept ${ }^{R 88 W / W t}$ mice could have been analyzed. In a next step, the abundance of SEPT9 in sciatic nerve lysates at P75 was analyzed on immunoblot level. In Addendum Figure 1B it is displayed, that the point mutation has no effect on the abundance of SEPT9. In the PNS the essential septin to form a filament is SEPT2. Accordingly, the abundance of SEPT2 was

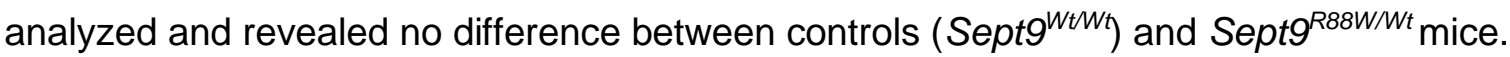


Taken together, the results suggest that the point mutation does not interfere with the presence of septins in PNS myelin.

A

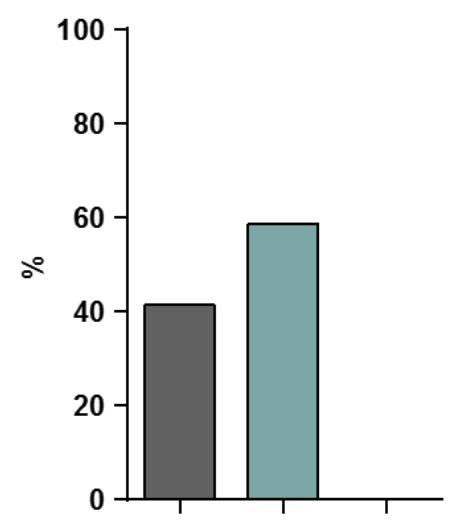

B

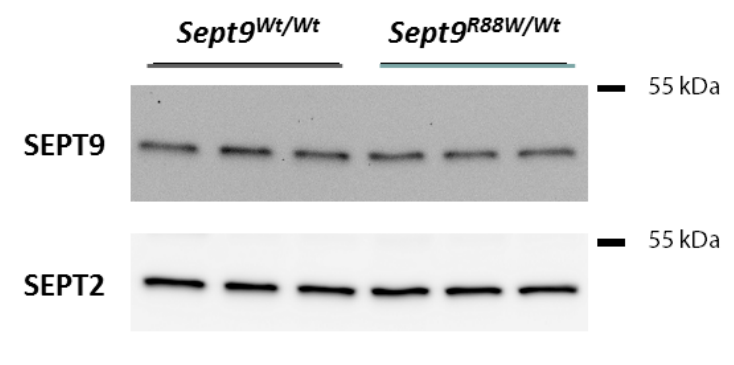

Addendum Figure 1: Offspring analysis and abundance of SEPT9 and SEPT2 in Sept9R88W/wt mice. (A) Offspring analysis of breedings with Sept9 ${ }^{R 88 W W W}$ males and Sept ${ }^{R 88 W / W t}$ females, depicts that no homozygous animals were born (Statistical expectation: 25\% WT; 50\% heterozygous; 25\% homozygous). In total, 121 pups were analyzed. (B) Immunoblot analysis of sciatic nerve lysates at P75 from control (Septg ${ }^{\text {t }} W_{\text {f }}$ ) and Sept9 ${ }^{R 88 W / W t}$ mice. SEPT9 and SEPT2 are not altered in Sept ${ }^{R 88 W / W t}$ mice.

\subsubsection{No changes in localization of SEPT9}

To assess whether the R88W point mutation has an effect on the localization of SEPT9, immunohistochemical stainings were performed on teased fibers of sciatic nerves at P75. There was no difference in SEPT9 localization detectable between Sept ${ }^{\text {Wt }}{ }^{\mathrm{W} t}$ and Sept ${ }^{R 88 W / W t}$ mice (Addendum Figure 2). In both genotypes, SEPT9 is located at nodes of Ranvier, bands of Cajal, and Schmidt-Lanterman incisures (SLI).

The results suggest that the point mutation has no effect on the localization of SEPT9 in sciatic nerves.
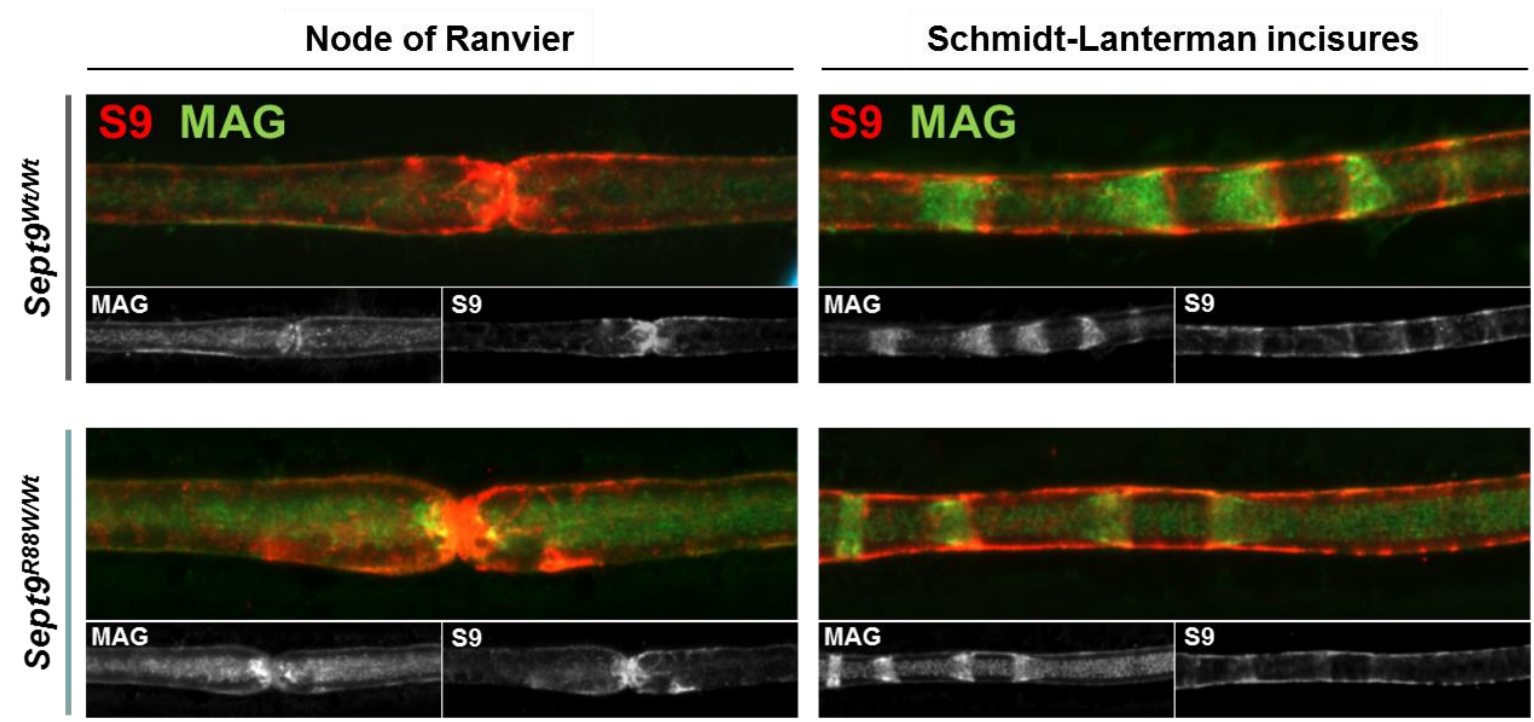

Addendum Figure 2: Localization of SEPT9 in sciatic nerves. Immunolabeling validates normal distribution and localization of SEPT9 (red) in teased fibers of sciatic nerves at P75. MAG (green) is stained as control, labeling the abaxonal and adaxonal myelin and SLIs. Labeling of SEPT9 reveals localization to nodes of 
Ranvier, abaxonal myelin, bands of Cajal, and SLIs. Images are representative of three independent experiments.

\subsubsection{Unaltered nerve structure}

To analyze the effect of the point mutation on the PNS, sciatic nerves were analyzed on semi-thin sections. Images were taken with a 100x oil objective of the Zeiss Axio Imager Z1 and stitched using the ZEN 2011 software (Addendum Figure 3A). Reconstruction reveals normal sciatic nerve structure at P75. Squares highlight respective regions within the sciatic nerves and indicate normal myelination. To further analyze whether the point mutation has an impact on myelination, myelinated axons were quantified and normalized to the nerve area (Addendum Figure 3B). The evaluation reveals normal numbers of myelinated axons within sciatic nerves of Sept ${ }^{\text {R88W/Wt }}$ mice compared to controls at P75.

The results indicate that the point mutation has no impact on the ability of Schwann cells to myelinate axons and therefore is also not altering the overall nerve structure.

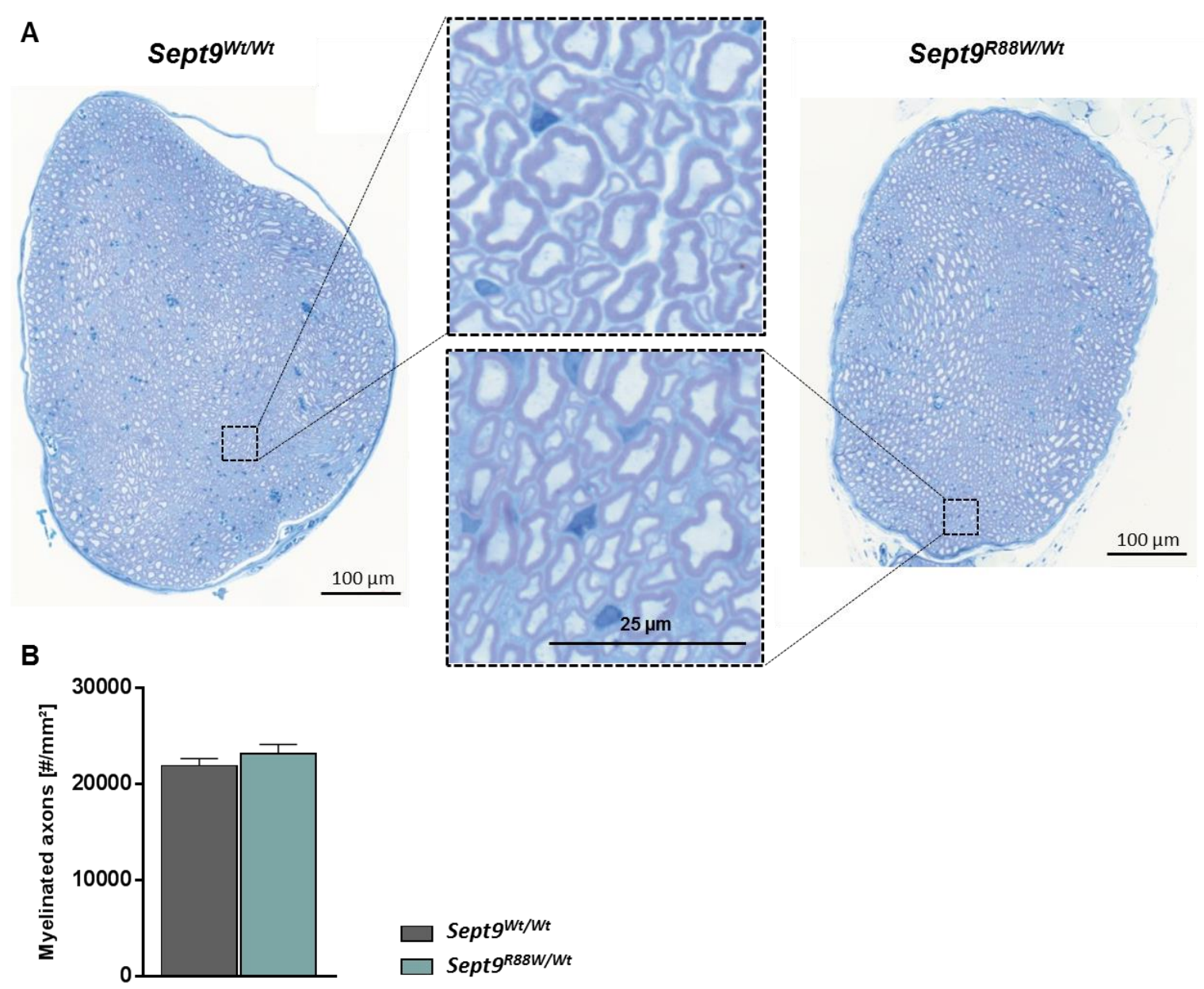

Addendum Figure 3: Nerve structure and myelinated axons. (A) Light microscopic images of Septg ${ }^{W t W t}$ and Sept9R88W/Wt sciatic nerves at P75. Squares highlight respective region. Images depict no sign of structural alterations in Sept ${ }^{R 88 W W W}$ mice. Images are representative for $\mathrm{n}=4$ animals per genotype. (B) Quantitative evaluation of all myelinated axons of light microscopic images of sciatic nerves at P75. Quantification reveals normal numbers of myelinated axons normalized to nerve area in Sept9R88W/Wt mice compared to controls. Mean +/ SEM. $n=4$ animals per genotype; not significant according to two-tailed unpaired t-test $(p=0.3311)$. 


\subsubsection{Normal myelin ultrastructure}

Sciatic nerves were also analyzed on electron microscopic level, to identify possible effects of the R88W point mutation on myelin ultrastructure or Remak bundle appearance. Electron micrographs (Addendum Figure 4) reveal normal myelinated axons and no alterations in myelin ultrastructure at P75 in Sept9 ${ }^{R 88 W W t}$ sciatic nerves. Additionally, the axons show no signs of pathology. Moreover, the images illustrate a normal size of Remak bundles. Within the Remak bundles, no alterations in axon numbers and axon size were found.

To sum these findings up, Sept $9^{R 88 W / W t}$ mice are able to form myelin in a normal state as control animals. There were no obvious ultrastructural changes detectable in Schwann cells of mice carrying the point mutation. Neither in myelinating Schwann cells, nor in Remak bundles.

Sept9wtwt

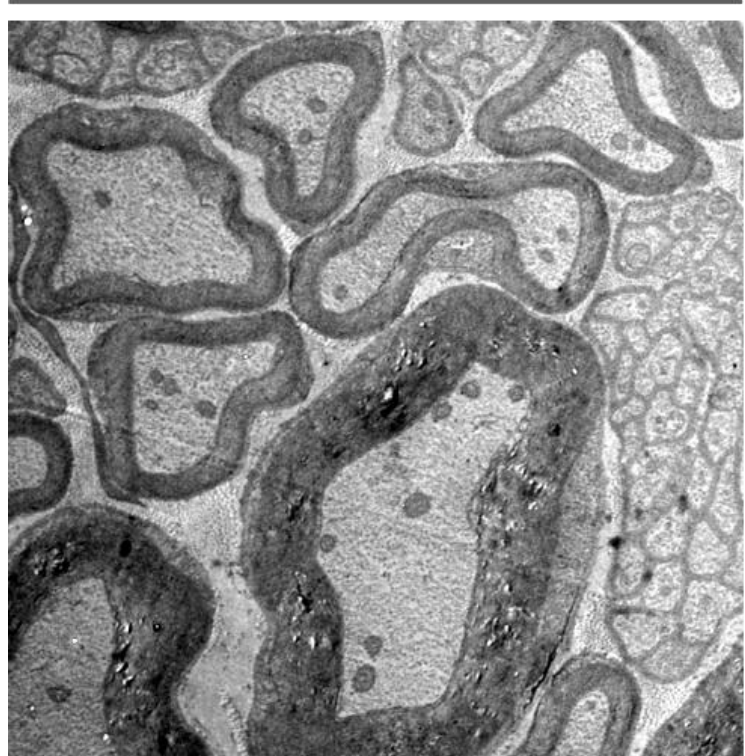

Sept9R88W/Wt

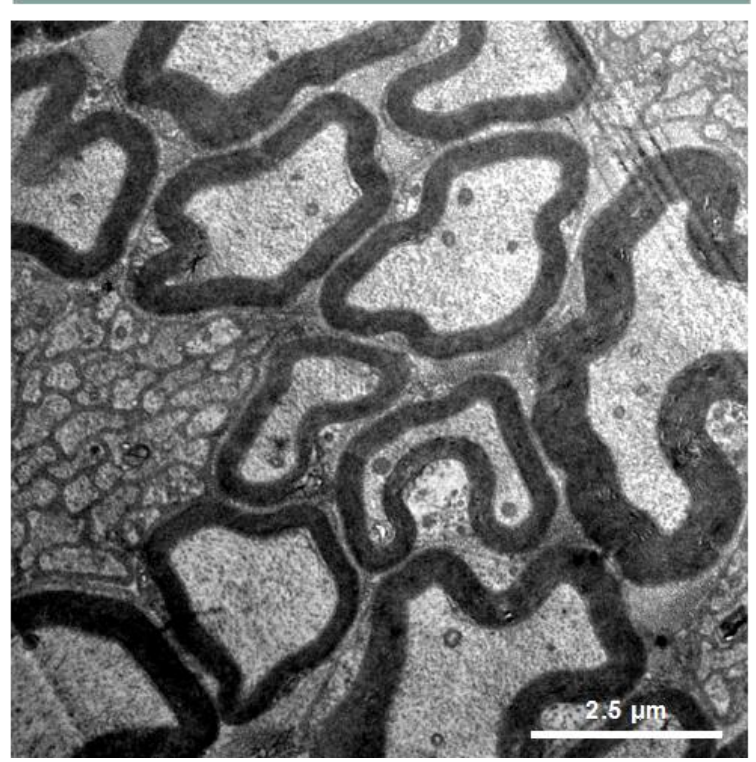

Addendum Figure 4: Myelin ultrastructure. Electron micrographs of control and Sept9R88W/Wt sciatic nerves at P75. Images illustrate normal myelination and healthy axons for both, controls (Septgwtwt) and Sept9R88W/Wt mice. Remak bundles appear normal in axon numbers and axon size. Images are representative for $n=4$ animals per genotype.

\subsubsection{Analysis of Sept ${ }^{f / / f l} D h h c+$ and Sept2 ${ }^{f / f l}{ }^{*} D h h c+$ mice}

\subsubsection{No alterations in axon sorting at $\mathbf{P} 4$}

To analyze the role of SEPT9 specifically for Schwann cells, Sept $9^{f / f|t|} D h h c+$ mice were analyzed. Additionally, Sept2 $2^{f / f t *} D h h c+$ mice were analyzed to identify the effect of the septin filament for Schwann cells. In Addendum Figure 5A, myelinated axons were analyzed at P4 in both, Sept $9^{f / f t *} D h h c+$ and Sept $2^{f / f t *} D h h c+$ sciatic nerves. The electron micrograph illustrates exemplary myelinated axons, which were considered for quantification. Axons that were enwrapped by at least one complete layer of compact myelin were considered myelinated. The quantification shows no difference in number of myelinated axons 
normalized to the analyzed area, neither by comparing Sept $t^{f / / t \mid}{ }^{t} D h h c+$ sciatic nerves to controls, nor comparing Sept2 ${ }^{f / / t *} D h h c+$ sciatic nerves to controls. As also non-myelinating Schwann cells lose the targeted gene upon recombination with $D h h$-Cre, the impact on axon sorting out of Remak bundles was analyzed in Sept ${ }^{f / / f t *} D h h c+$ and Sept2 ${ }^{f / f / *} D h h c+$ sciatic nerves (Addendum Figure 5B). The electron micrograph illustrates exemplary out-sorted axons, which were considered for quantification. Axons that were enwrapped by a Schwann cell in a one to one ratio, and axons that were partially enwrapped by compact myelin, were considered out-sorted. The quantification reveals no difference in number of out-sorted axons normalized to the analyzed area, between mutants and their respective controls.

The results indicate that both, SEPT9 and the presence of all septins, are not required to properly myelinate and out-sort axons in young mice.

A
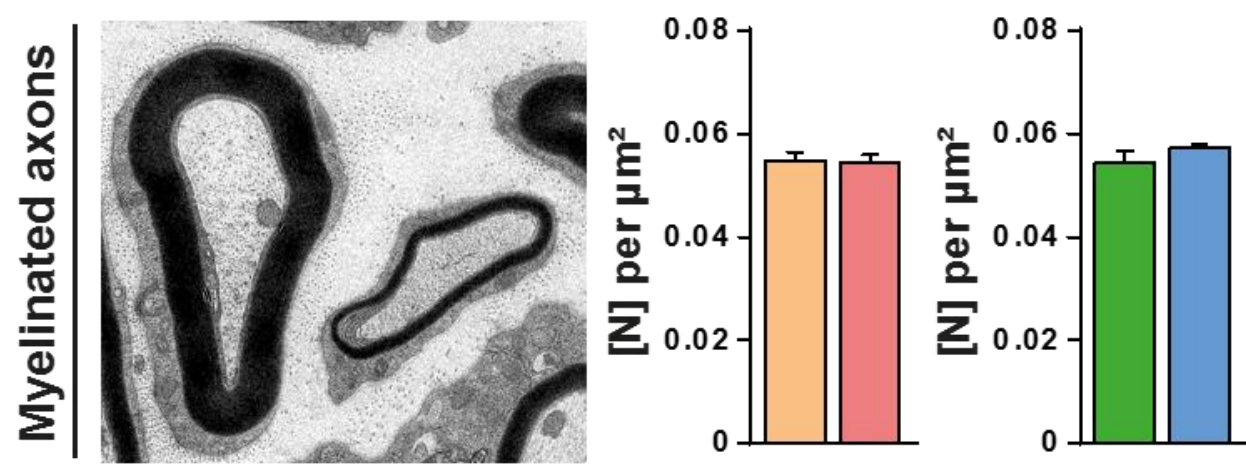

B
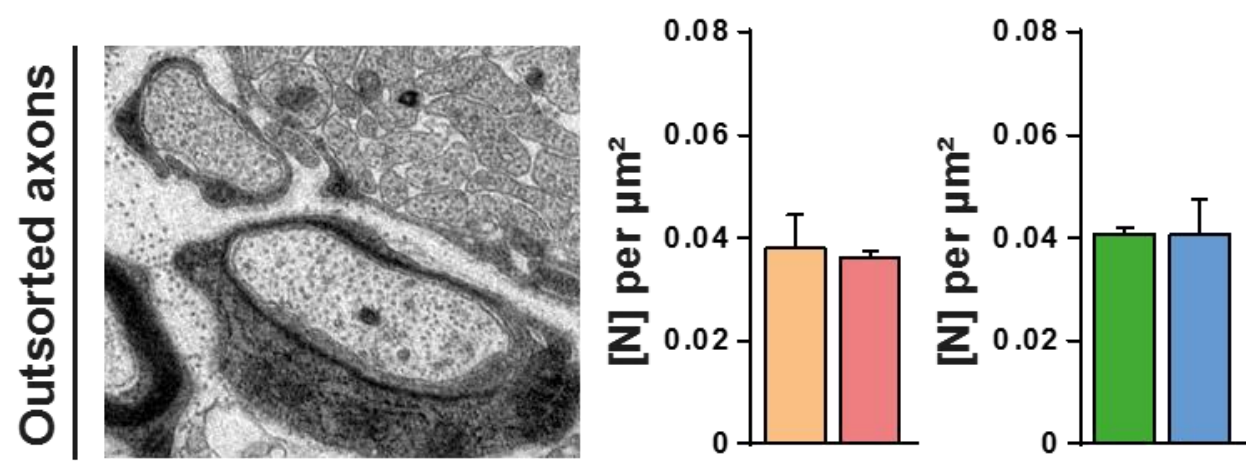

Sept9f//f

Sept $9^{\text {ff/f*}}$ Dhhc+

Sept2 $2^{\text {f/f }}$

Sept2 ${ }^{f / f f}$ Dhhc+

Addendum Figure 5: Sorting and myelination in Sept $9^{f / f l}{ }^{*} D h h c+$ and Sept2 ${ }^{f / f f}$ Dhhc+ mice at P4. (A) Quantitative evaluation of myelinated axons of Sept $9^{f / f t *} D h h c+$ and $S e p t 2^{f / f / *} D h h c+$ sciatic nerves at P4. Electron micrograph illustrates examples of quantified myelinated axons. Mean +/ SEM. $n=3-4$ animals per genotype; not significant according to two-tailed unpaired t-test. (B) Quantitative evaluation of out-sorted axons of Sept9f//ft*$D h h c+$ and Sept $2^{f / f / t *} D h h c+$ sciatic nerves at P4. Electron micrograph illustrates examples of quantified out-sorted axons. Mean +/ SEM. $n=3-4$ animals per genotype; not significant according to two-tailed unpaired t-test. 


\subsubsection{Number of myelinated axons are unchanged at P14}

The myelination status at P14 was analyzed in Sept ${ }^{f / f t *} D h h c+$ and Sept2 $2^{f / f t *} D h h c+$ sciatic nerves (Addendum Figure 6) to see whether the loss of SEPT9 or all septins lead to any impairments. All myelinated axons in sciatic nerves were analyzed and normalized to the respective nerve size. The upper row of Addendum Figure 6 depicts the quantification for SEPT9 conditional mutants and a light microscopic image of a mutant nerve. The highlighted square indicates an area of higher magnification of the sciatic nerve semi-thin section, which reveals normal appearing myelinated axons. The quantification of myelinated axons per $\mu \mathrm{m}^{2}$ shows no difference between Sept ${ }^{f / / f t}{ }^{*} D h h c+$ mice and their respective controls. The lower row reveals the quantification for SEPT2 conditional mutants and a light microscopic image of a mutant nerve. The highlighted square indicates an area of higher magnification of the sciatic nerve semi-thin section, depicting normal appearing myelinated axons. The quantification of myelinated axons per $\mu \mathrm{m}^{2}$ displays no difference between Sept $2^{f / f t *} D h h c+$ mice and their respective controls.

The results suggest that neither the loss of SEPT9, nor the loss of all Schwann cell septins lead to impaired myelination at P14. Moreover, it is indicated that septins in the PNS have no effect on the developmental myelination.

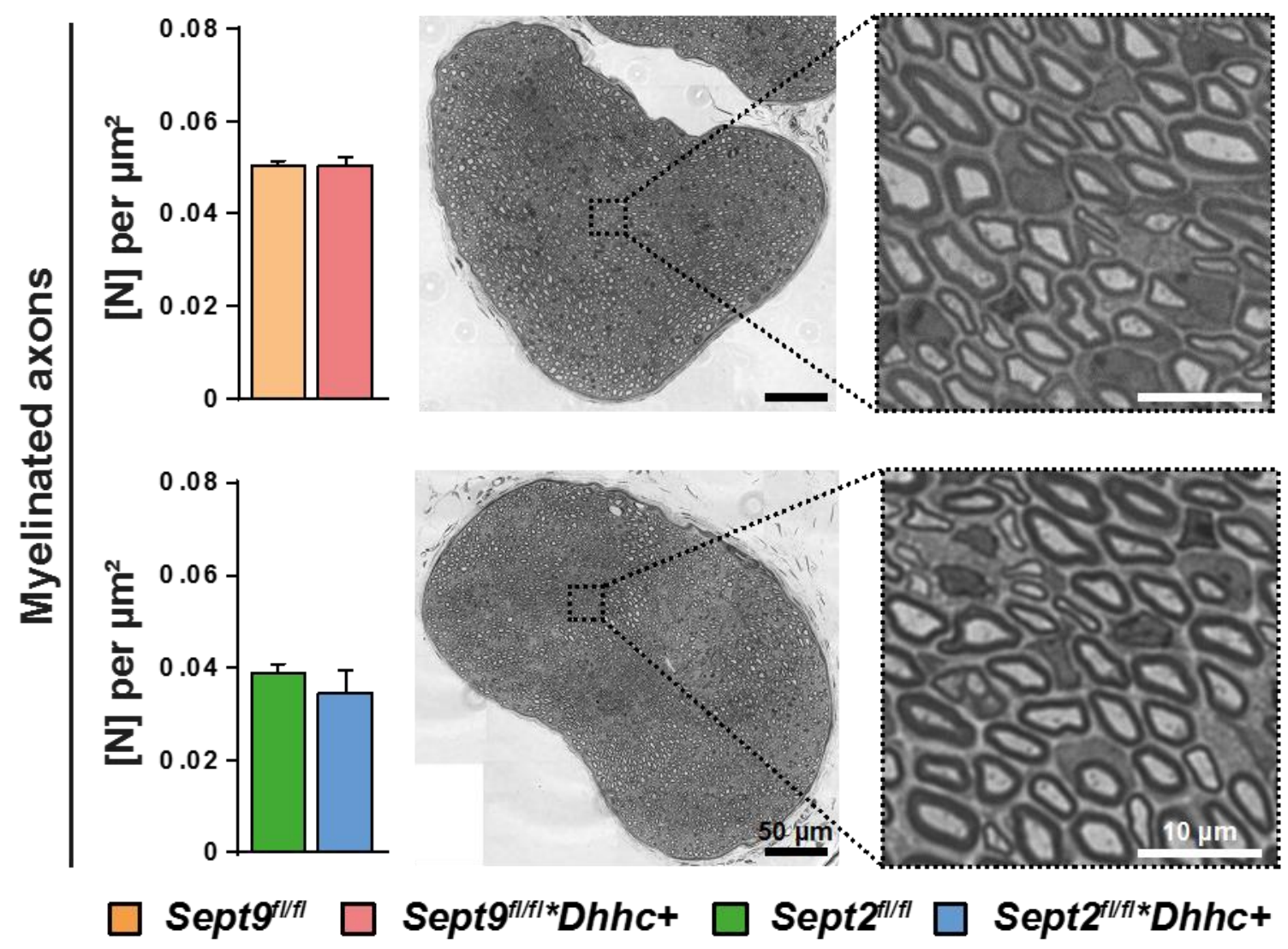

Addendum Figure 6: Myelinated axons at P14. Quantitative evaluation of myelinated axons of Sept $9^{f / f t *} D h h c+$ and Sept2 $2^{f / f t} D h h c+$ sciatic nerves at P14. Light micrograph illustrates example of a mutant sciatic nerve

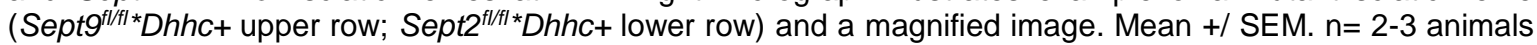
per genotype; not significant according to two-tailed unpaired t-test. 


\subsubsection{Normal myelination state at 6 mo of age}

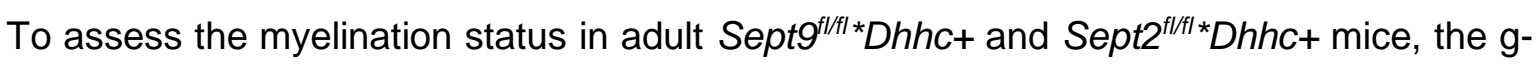
ratio was analyzed at $6 \mathrm{mo}$ of age in sciatic nerves (Addendum Figure 7). The quantitative analysis reveals no differences in g-ratio between Septg ${ }^{f / t / t} D h h c+$, Sept2 ${ }^{f / t / *} D h h c+$, and their respective controls, suggesting that the loss of SEPT9 or PNS myelin septins have no effect on the maintenance of myelin.

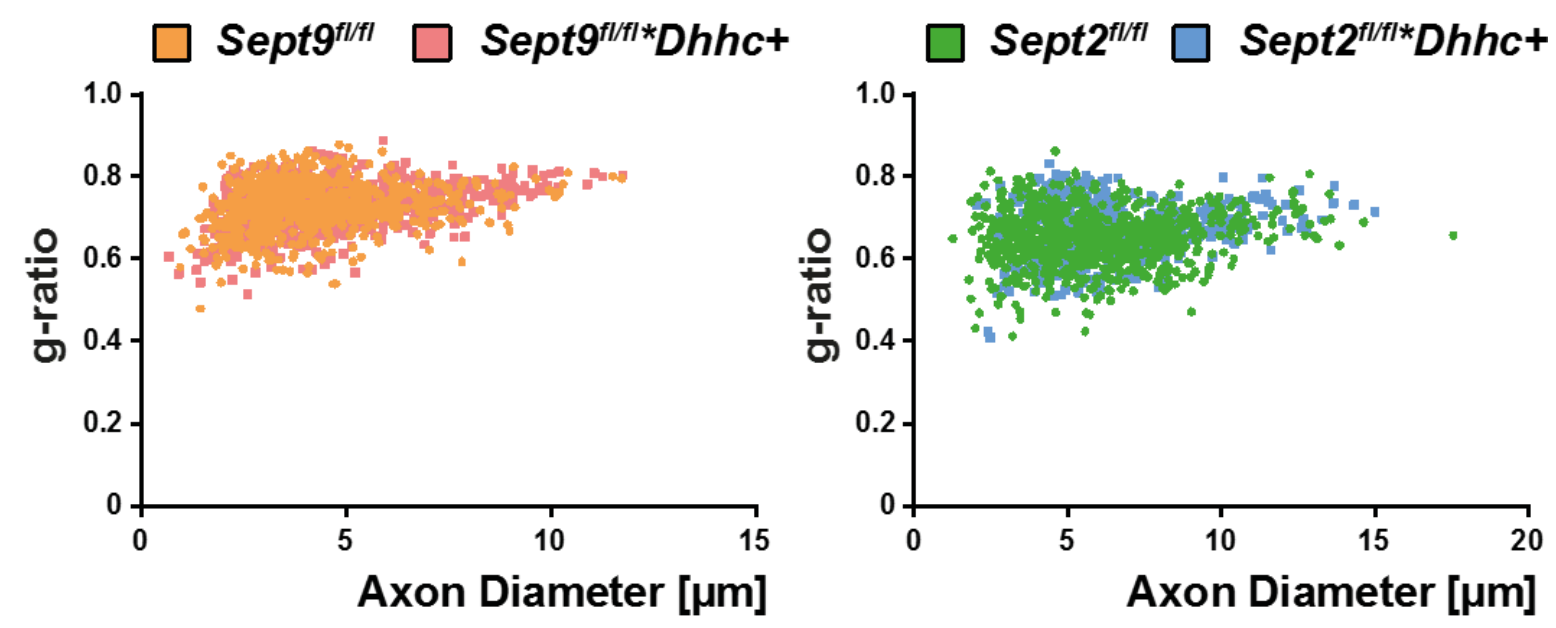

Addendum Figure 7: g-ratio in Sept ${ }^{f / / f l}{ }^{*} D h h c+$ and Sept $2^{f / f l}{ }^{*} D h h c+$ mice. g-ratio analysis of electron micrographs of sciatic nerves at 6 mo of age indicates normal myelin sheath thickness in Sept9t/fi*Dhhc+ and Sept $2^{f / f t *} D h h c+$ mice. $\mathrm{n}=4$ animals per genotype.

\subsubsection{No changes in axon diameters Septg ${ }^{f / f f t}$ Dhhc+ mice}

As it was shown that septin deficient mice depict increased axon diameters in the CNS, the axon diameter frequency distribution in Sept ft/ft*$^{\prime} D h h c+$ mice was analyzed (Addendum Figure 8). For the analysis, the brachial plexus was chosen, because this nerve is mostly affected in human patients. No difference in the axon diameter distribution were found between controls and Sept ${ }^{t / f / t}{ }^{*} D h h c+$ mice. This was also indicated by the analysis of all axon diameters with the Kolmogorov Smirnov test.

To summarize these results, loss of SEPT9 in Schwann cells does not lead to alterations of axon diameters, which suggests that the loss of SEPT9 does not lead to alterations in axon diameter in the PNS. 

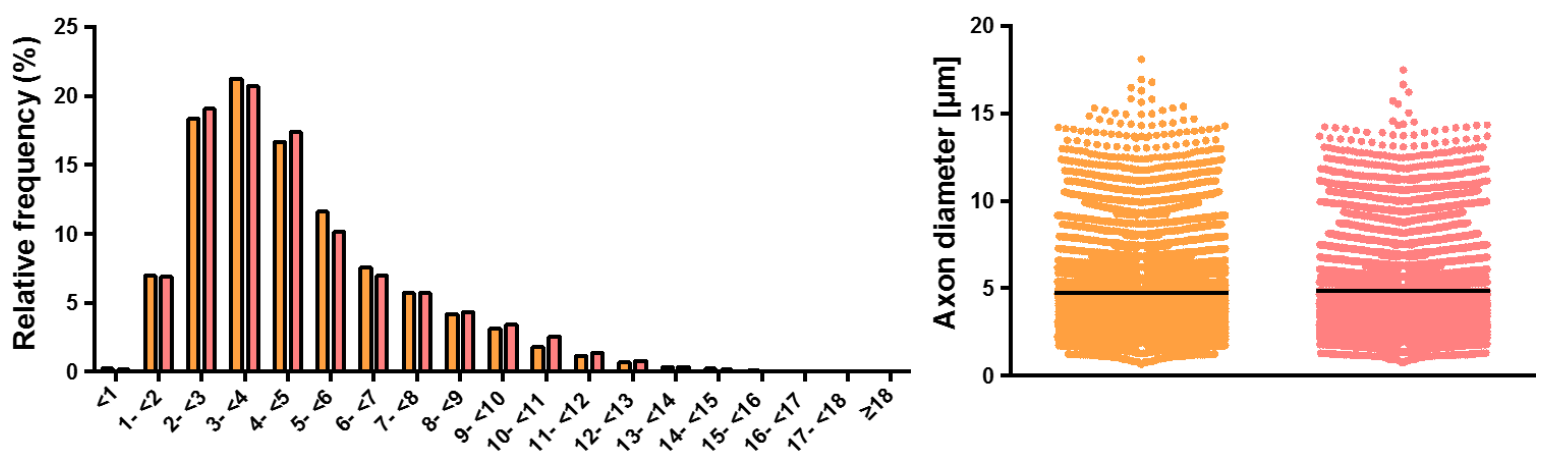

Addendum Figure 8: Axon diameter frequency distribution in Septgf/f/*Dhhc+ mice. Frequency distribution analysis of myelinated axons in the brachial plexus of electron micrographs at 6 mo of age indicate no shift in axon diameter in Sept9f/f/*$D h h c+$ mice. $n=4$ animals per genotype. Not significant according to Kolmogorov Smirnov test $(p=0.1746)$.

\subsection{Perspective}

The results of the performed experiment identified no significant difference between control and the different mutant mice. In previous studies, Sept2 and Sept9 cKO mice were analyzed more broadly (unpublished data). This analysis showed no difference in both mutants compared to controls in several different analysis including immunohistochemical labeling, behavioral tasks measuring motor and sensory capabilities, and assessment of myelin ultrastructure (unpublished data). If the emergence of HNA is related to external stress is not known. Thus, it would be interesting to challenge the three different mutants for instance by nerve compression. Similar aspects were described for other neuropathies, where conduction blocks led to the formation of outfoldings (Bai et al., 2010). It is still under debate whether inflammation leads to the observed phenotype in HNA patients. To analyze this aspect, it would be interesting to induce inflammation in the PNS to activate the immune system (Wang Ip et al., 2006). So far, it is known that also partial gene duplications lead to HNA (Kuhlenbaumer et al., 2005). Therefore, mice overexpressing SEPT9, wild type or mutated, might be necessary to gain a genuine model for the disease.

Although lacking deeper knowledge on the disease HNA, it appears that septins seem to have different roles in CNS and PNS myelin. Although oligodendrocytes and Schwann cells form compact myelin, it seems that the mechanisms of maintaining functional compact myelin are different. It is known that the protein composition is different in oligodendrocytes and Schwann cells (Nave and Werner, 2014), as these are obviously different cell types. Nevertheless, it is remarkable that septins have a similar distribution in both cell types, but seem to have different functions. 